hep-th/9702194

February 1997

\title{
LECTURES ON CONFORMAL FIELD THEORY AND KAC-MOODY ALGEBRAS
}

\author{
Jürgen Fuchs ${ }^{x}$ \\ DESY \\ Notkestraße 85 \\ D - 22603 Hamburg
}

\begin{abstract}
.
This is an introduction to the basic ideas and to a few further selected topics in conformal quantum field theory and in the theory of Kac-Moody algebras. These lectures were held at the Graduate Course on Conformal Field Theory and Integrable Models (Budapest, August 1996). They will appear in a volume of the Springer Lecture Notes in Physics edited by Z. Horvath and L. Palla.
\end{abstract}

X Heisenberg fellow 


\section{Contents}

Lecture 1 : Conformal Field Theory 3

1 Conformal Quantum Field Theory 3

2 Observables: The Chiral Symmetry Algebra

3 Physical States: Highest Weight Modules

4 Sectors: The Spectrum 9

5 Conformal Fields 11

6 The Operator Product Algebra 14

7 Correlation Functions and Chiral Blocks 16

Lecture 2: Fusion Rules, Duality and Modular Transformations

8 Fusion Rules 19

9 Duality 21

10 Counting States: Characters 23

11 Modularity 24

12 Free Bosons 26

13 Simple Currents

14 Operator Product Algebra from Fusion Rules 30

Lecture 3: Kac-Moody Algebras 32

15 Cartan Matrices 32

16 Symmetrizable Kac-Moody Algebras 35

17 Affine Lie Algebras as Centrally Extended Loop Algebras 36

18 The Triangular Decomposition of Affine Lie Algebras 38

19 Representation Theory 39

20 Characters

Lecture 4: WZW Theories and Coset Theories 42

21 WZW Theories 42

22 WZW Primaries 43

23 Modularity, Fusion Rules and WZW Simple Currents 44

24 The Knizhnik-Zamolodchikov Equation 46

25 Coset Conformal Field Theories 47

26 Field Identification 48

27 Fixed Points

28 Omissions 51

29 Outlook 52

30 Glossary 53

References 55 


\section{Lecture 1: Conformal Field Theory}

\section{Conformal Quantum Field Theory}

Over the years, quantum field theory has enjoyed a great number of successes. Nevertheless, from a conceptual point of view the situation is far from satisfactory, and in some respects it even resembles a disaster. Indeed, beyond perturbation theory many of the methods used in phenomenologically successful models cannot be justified rigorously. For instance, a thorough understanding of path integrals is essentially only available for free fields, or for topological 'field' theories which have a finite-dimensional configuration space. Also, the relation between the elementary 'point-like' quantum fields (whatever the precise meaning of such quantities may be) and the actual particle contents of a theory is often obscure. (The elementary fields may be regarded as a coordinatization of the field space, while the particles have a meaning independent of a choice of coordinates; typically a distinguished and / or manageable choice of field coordinates which directly correspond to particles is not available.) On the other hand, frameworks whose foundations are mathematically sound, like e.g. Wightman field theory [104] or $C^{*}$-algebraic approaches [70], are difficult to relate to concrete models, and indeed have traditionally been plagued with a scarcity of models to which they apply. For example, the proper treatment of $U(1)$-charges (which are coulombic rather than localized) in the framework of algebras of local observables is still problematic.

It is worth stressing that 'quantum field theory' is not a protected term, but is used for a variety of rather different concepts. In particular there are two basically different points of view of the relation between 'quantum' versus 'classical' field theories. One may either try to quantize a classical theory (say by canonical quantization or using path integrals), or directly start at the quantum level, e.g. by describing the field and observable algebras, in which case a classical theory may be obtained by performing a suitable limit. It is far from obvious that the resulting quantum, respectively classical, theory is uniquely determined. Another important difference concerns the description of observables. In some approaches these are characterized quite concretely (e.g. as gauge respectively BRS invariant combinations of the elementary field variables of the classical theory), in others they appear more abstractly as operators on a Hilbert space which at an initial stage are only known via their general algebraic properties.

Conformal field theory admits formulations which directly approach the exact theory at the quantum level, but nevertheless describe observables quite explicitly and handle large classes of models simultaneously. In particular many quantities of interest can be calculated exactly rather than only in a (typically at best asymptotic) perturbation series. In addition, for a large number of models there exists (or is at least conjectured) a Lagrangian/path integral realization, so that in principle one can study such models in several guises and e.g. compare with conventional perturbation theory. Conformal field theory can therefore help to bridge the gap between various approaches to quantum field theory.

Here and below, by the term conformal field theory I refer to models in one or two spacetime dimensions which are relativistic quantum field theories and possess conformal symmetry. What precisely this characterization means will be studied shortly. Again one must note that

${ }^{1}$ While a lot of physicists believe to know exactly what quantum field theory is, you will most probably get two different answers when you ask any two of them. And not only the methods and results are under debate, but even the basic questions to be asked. 
the term 'conformal field theory' is not protected and can stand for a variety of different formulations. Here I approach this subject from the perspective of quantum field theory. However, in this introductory exposition I will not attempt to build exclusively on general field theoretic considerations, but rather I concentrate on certain basic algebraic structures and assume that the reader has already some faint acquaintance with fundamental field theoretic notions that will play a rôle, such as the operator product algebra, correlation functions and fusion rules. On the other hand, in a sense I start from scratch, in that at least I try to indicate each logical step explicitly, even though I probably do not provide sufficient explanation for all of them.

The motivations to study conformal field theories are manifold, and I am content to just drop a few keywords here: f field theories describing systems at the critical point of a second order phase transition in statistical mechanics; vacuum configurations of strings and superstrings; braid group statistics; topological field theory; invariants of knots and links, and of threemanifolds; integrable systems as perturbed conformal models; the fractional quantum Hall effect; high temperature superconductivity.

In the study of conformal field theories, the following two issues prove to be most fundamental. First, the classification programme; and second, the complete solution, by reconstruction from certain basic data (possibly obtained in the course of classification), of specific models. We will encounter various manifestations of these two aspects of conformal field theory in the sequel. They indicate that these theories are very special indeed: while (contrary to the impression that is occasionally given) a classification of all conformal field theories or a complete solution of any arbitrary given conformal field theory model is totally out of reach, one can classify large classes of conformal field theories, and one can solve very many models to a large extent.

\section{Observables: The Chiral Symmetry Algebra}

One of the ultimate goals of a physical theory is to predict the outcome of all possible measurements. Accordingly, a basic step in the investigation of a conformal field theory consists of characterizing its observables. In quantum physics, one thinks of observables as operators acting on a Hilbert space of physical states. Moreover, in relativistic quantum field theory it is usually necessary to consider operators which are bounded (only these can be continuous) and are localized in some compact space-time region, i.e. act as the identity outside [70]. Observables localized in space-like separated regions commute (locality principle). In contrast, below I will work with non-localized unbounded operators; that this does not lead to any severe problems has its origin in the fact that these observables enjoy an underlying Lie algebra structure. To obtain localized bounded operators one would, roughly speaking, have to smear these operators with test functions of compact support and form bounded functions thereof.

The most important subset of these observables of a conformal field theory is provided by the conformal symmetry itself. Conformal transformations of space-time - i.e. of a (pseudo-)Riemannian manifold - are by definition those general coordinate transformations which preserve the angles between any two vectors, or what is the same, which scale the metric locally by an over-all factor (Weyl transformations). In a flat space-time of $D>2$ dimensions the infinitesimal conformal transformations consist of translations, rotations (respectively

\footnotetext{
${ }^{2}$ For more details I refer to the references listed in section 28 , to other lectures delivered at this school, as well as to the huge number of works where such motivations are advertised.
} 
Lorentz boosts), a dilation and so-called special conformal transformations; these generate a $(D+1)(D+2) / 2$-dimensional Lie algebra, which is a real form of $\mathfrak{s o}(D+2, \mathbb{C})($ e.g. $\mathfrak{s o}(D, 2)$ in the case of Minkowski space). P In contrast, for $D=2$ any arbitrary holomorphic mapping of the (compactified) complex plane is angle-preserving. Thus in complex coordinates $z, \bar{z}$, infinitesimal conformal transformations are generated by mappings which transform $z$ as $z \mapsto z+\eta(z)$ by functions which do not depend on $\bar{z}-$ a basis of which is given by $\eta(z)=-z^{n+1} \epsilon$ for $n \in \mathbb{Z}$ - and by analogous mappings of $\bar{z}$ with functions which do not depend on $z$. On functions of $z, \bar{z}$ these mappings are generated by differential operators

$$
l_{n}=-z^{n+1} \partial_{z} \quad \text { and } \quad \bar{l}_{n}=-\bar{z}^{n+1} \partial_{\bar{z}},
$$

respectively. The operators $l_{n}$ satisfy the commutator relations

$$
\left[l_{m}, l_{n}\right]=(m-n) l_{m+n}
$$

and an analogous formula holds for the $\bar{l}_{n}$. In mathematical terms, this means that both the $l_{n}$ and the $\bar{l}_{n}$ span an infinite-dimensional Lie algebra; moreover, these two algebras are combined as a direct sum, i.e. one has $\left[l_{m}, \bar{l}_{n}\right]=0$. The Lie algebra defined by (1.2) is known as the Witt algebra.

The formula (1.2) was obtained by purely classical considerations. In quantum theory, there are analogous operators $L_{n}$ with $n \in \mathbb{Z}$, but now they satisfy

$$
\left[L_{m}, L_{n}\right]=(m-n) L_{m+n}+\frac{1}{12}\left(m^{3}-m\right) \delta_{m+n, 0} C .
$$

That is, they again give rise to a Lie algebra, but there appears an additional generator $C ; C$ is a central element, i.e. has zero Lie bracket with the whole algebra,

$$
\left[C, L_{n}\right]=0
$$

The infinite-dimensional Lie algebrat defined by (1.3) and (1.4) is called the Virasoro algebra and denoted by $\mathcal{V i r}$. In short, the Lie brackets of $\mathcal{V} \mathfrak{i r}$ differ from their classical counterpart only in the term with $C$; the generator $C$, which in mathematics is known as the canonical central element or central charge, is therefore in physics also referred to as the conformal anomaly. As in the classical situation one deals in fact with the direct sum of two Virasoro algebras, the second one being generated by operators $\bar{L}_{n}, n \in \mathbb{Z}$, and $C$.

In a purely mathematical context, the relations (1.3) arise - by solving a cohomology problem [53] - as the unique non-trivial central extension of (1.2). Field-theoretically, (1.3) is obtained by making a general ansatz for the equal-time commutator of the 'energy-momentum tensor', whose Fourier-Laurent components are the operators $L_{n}$ and $\bar{L}_{n}$ (see equation (1.25) below). When one imposes the requirements that the Wightman axioms are satisfied, that the system is dilation invariant, and that the energy-momentum tensor is a conserved (Noether)

${ }^{3}$ More generally, the real form is $\mathfrak{s o}(p+1, q+1)$ for signature $\left((+)^{p},(-)^{q}\right)$.

4 Let me remind you at this point that, by definition, a Lie algebra over $\mathbb{C}$ (or similarly over $\mathbb{R}$, or more generally over any field of scalars $\{\xi\}$ ), is a vector space with a bilinear product - called the Lie bracket and denoted by $[\cdot, \cdot]$ - which is antisymmetric and satisfies the Jacobi identity. In physics, the most common realization of a Lie bracket is as the commutator with respect to an underlying associative product.

5 Also note: That the energy-momentum tensor is both conserved and traceless implies that the 'dilation currents' $x^{\mu} T_{\mu \nu}$ are conserved. 
current, this equal-time commutator becomes a finite sum of products of (derivatives of) the $\delta$ function multiplied with local operators, and this result - known as the Lüscher-Mack theorem [57] - is equivalent to (1.3).

Let me point out once more that in a quantum field theoretic setting the physical meaning of the generators of conformal symmetry is as providing (non-bounded, non-localized) observables, in the same spirit as e.g. momentum and angular momentum are observables in ordinary one-particle quantum mechanics. A brief characterization of a conformal field theory is therefore that the observable algebra contains (the direct sum of two copies of) the Virasoro algebra. In general, the full observable algebra of a conformal field theory may be larger, though. But by considerations based on properties of conserved currents, similarly as in the proof of the Lüscher-Mack theorem, one can argue that the observables (regarded as Fourier-Laurent components of conserved currents) still generate a Lie algebra, and the direct sum structure persists as well. In short, the observables of a conformal field theory form an infinite-dimensional Lie algebra $\mathcal{W}_{\text {tot }}$ over $\mathbb{C}$ with countable basis, which can be written as

$$
\mathcal{W}_{\text {tot }}=\mathcal{W} \oplus \overline{\mathcal{W}}^{\prime} \quad \text { with } \quad \mathcal{W} \supset \mathcal{V i r} \quad \text { and } \quad \overline{\mathcal{W}}^{\prime} \supset \overline{\mathcal{V} \mathfrak{i r}}
$$

There is a variety of names for the two direct summands $\mathcal{W}$ and $\overline{\mathcal{W}}^{\prime}$ : the holomorphic / antiholomorphic, or chiral / antichiral, or left/right subalgebras. When one considers only one of these subalgebras, one refers to the observables as the chiral symmetry algebra. As a consequence of the direct sum structure, for many purposes one can restrict ones attention to one 'chiral half' of the two-dimensional theory, and I will do so for the time being. (For aspects of the two-dimensional theory see e.g. sections 7,9,14 below. Also note that one needs not necessarily require that the left and right chiral halves have isomorphic chiral symmetry algebras; when $\overline{\mathcal{W}}^{\prime} \neq \mathcal{W}$, then one speaks of a 'heterotic' theory.)

Unless stated otherwise, from now on it will be assumed that $\mathcal{W}$ is maximal, i.e. that it already includes all (chiral) observables. $\mathcal{W}$ has a basis of the form $\left\{W_{n}^{i}\right\} \cup\left\{C_{\ell}\right\}$, where the index $n$ of $W_{n}^{i}$ takes values in $\mathbb{Z}$, while the index $i$ takes values in a set $I \equiv\{0,1,2, \ldots\}$ which may be finite or possibly infinite, and where the $C_{\ell}$ are central elements satisfying $\left[C_{\ell}, \cdot\right]=0$. The Virasoro generators can be identified as $L_{n} \equiv W_{n}^{0}$. At least for $m \in\{0, \pm 1\}$ any generator $W_{n}^{i}$ then satisfies

$$
\left[W_{m}^{0}, W_{n}^{i}\right] \equiv\left[L_{m}, W_{n}^{i}\right]=\left(\left(\Delta_{i}-1\right) m-n\right) W_{m+n}^{i},
$$

with certain numbers $\Delta_{i}$ which must be positive integers (on the other hand, for $i=0$ and $m \neq\{0, \pm 1\}$, there is in addition the central term). In particular, the subscript $n$ already determines the Lie bracket with the Virasoro algebra generator $L_{0}$,

$$
\left[L_{0}, W_{n}^{i}\right]=-n W_{n}^{i} .
$$

In fact, this subscript even supplies us with a $\mathbb{Z}$-grading of $\mathcal{W}$, i.e. the structure constants of $\mathcal{W}$, defined by $\left[W_{m}^{i}, W_{n}^{j}\right]=\sum_{p \in \mathbb{Z}} \sum_{k \in I} f_{m n, k}^{i j, p} W_{p}^{k}$ are subject to the condition

$$
f_{m n, k}^{i j, p}=0 \quad \text { for } \quad p \neq m+n .
$$

\footnotetext{
${ }^{6}$ In principle, on the right hand side infinitely many terms may appear. In this case strictly speaking one does not deal with a proper basis of a Lie algebra, and a completion with respect to a suitable topology is needed. However, as it turns out, when applied to any vector in the relevant space $\mathcal{H}$ of physical states, only a finite number of terms is non-zero, owing to the fact that the generators $W_{m}^{i}$ act 'locally nilpotently' in $\mathcal{H}$. This is also one of the reasons why considering formally infinite series as in (1.24) below does not lead to any serious problems.
} 
Also, central elements have grade 0 .

For the time being I will not be more specific about the algebra $\mathcal{W}$. Later on, we will encounter important examples, the so-called current algebras. Other possibilities are supersymmetric extensions of the Virasoro algebra, and many more chiral algebras are described in detail in the lectures by Gerard Watts.

Before proceeding, let me point out that the restriction to one chiral half requires in particular to regard $z$ and $\bar{z}$ as two independent complex variables; $\bar{z}$ is to be identified with the complex conjugate of $z$ only once the two chiral halves of a two-dimensional theory are combined. That this is a sensible way to proceed is far from obvious, and actually the interpretation of this prescription depends in part on the context in which the conformal symmetry is considered:

- When arising in minkowskian quantum field theory, the variables $z$ and $\bar{z}$ take their values on circles that are obtained as the two compactified light-cones $x^{0}= \pm x^{1}$ of the two-dimensional theory, from which they are extended to the punctured plane $\mathbb{C} \backslash\{0\}$. Identification of $\bar{z}$ as the conjugate of $z$ thus amounts to considering an analytical continuation of the original minkowskian theory to euclidean signature which does not coincide with the usual Wick rotation.

- In applications to string theory, the punctured plane arises as the image of the cylindrical world sheet swept out by a closed relativistic string. In the description of phase transitions, $z$ is the complex coordinate of a euclidean two-dimensional field theory. The meaning of separating $z$ and $\bar{z}$ is in these cases less clear.

- At the level of the chiral algebra, taking $z$ and $\bar{z}$ as independent amounts to regarding $\mathcal{W}$ and $\overline{\mathcal{W}}$ as algebras over the complex numbers. It is tempting to interpret the identification of $\bar{z}$ with the complex conjugate of $z$ as considering a suitable real form of the complex Lie algebra, but this is actually not quite correct.

\section{Physical States: Highest Weight Modules}

Once we know the observables, the next logical step is to investigate how they 'act' on a space $\mathcal{H}$ of physical states. Ideally, this way in the end we describe all properties of a conformal field theory model in terms of its (maximal) chiral symmetry algebra $\mathcal{W}$. Now in the conventional operator approaches to quantum field theory, $\mathcal{H}$ is a separable Hilbert space and the observables are contained in the algebra of bounded linear operators on this Hilbert space. As already pointed out, here we are in a somewhat different situation. Nevertheless, the following basic requirements are still implicit in the concept of a space of physical states:

(H1) $\mathcal{H}$ is a representation space of the observable algebra, i.e. of $\mathcal{W}$.

In the following I will employ the shorter term module common in mathematics as a synonym for 'representation space'; elements of $\mathcal{H}$ are denoted by $v, w, \ldots$.

(H2) The representation of $\mathcal{W}$ on $\mathcal{H}$ is unitary. Correspondingly, $\mathcal{H}$ is endowed with a positive hermitian product $(\cdot \mid \cdot)$, so that it becomes a pre-Hilbert space.

(H3) The spectrum condition: The energy is bounded from below.

In order that the qualification 'unitary' in property (H2) makes sense, $\mathcal{W}$ must be endowed

7 The extension is by normal ordered products of supercurrents; the supercurrents themselves have half integral $\Delta$, i.e. are not observables and hence are not contained in $\mathcal{W}$. 
with a generalized complex conjugation, i.e. a mapping $x \mapsto x^{*}$ satisfying

$$
\left(x^{*}\right)^{*}=x, \quad(\xi x)^{*}=\bar{\xi} x, \quad(x y)^{*}=y^{*} x^{*}
$$

(in mathematical terms: $\mathcal{W}$ must be a ${ }^{*}$-algebra), such that

$$
(v \mid x w)=\left(x^{*} v \mid w\right)
$$

holds for the hermitian product on $\mathcal{H}$. In the last of the relations (1.9) (and, likewise, often implicitly below), one works in fact within the universal enveloping algebra of $\mathcal{W}$, i.e. with the associative algebra $\mathfrak{U}(\mathcal{W})$ that (roughly) can be described as consisting of formal products of elements of $\mathcal{W}$, with the Lie bracket of $\mathcal{W}$ equal to the commutator in $\mathfrak{U}(\mathcal{W})$.

In formulating the spectrum condition (H3), it is in particular assumed that among the observables there is an energy operator. In chiral conformal field theory this operator is provided by the Virasoro generator $L_{0}$, as follows from the rôle played by $L_{0}$ in applications. Briefly, $L_{0}$ acts as $-z \frac{\partial}{\partial z}$ and hence measures the mass (or scaling) dimension, i.e. $L_{0} \mathcal{O}=\Delta \cdot \mathcal{O}$ for operators

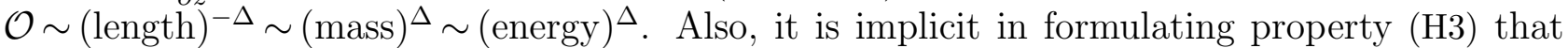
the action of $L_{0}$ on $\mathcal{H}$ can be diagonalized, so that $\left(L_{0}\right)^{*}=L_{0}$ by (H2). The eigenvalue $\Delta$ of $L_{0}$ on an eigenvector in $\mathcal{H}$ is called the conformal dimension or the conformal weight of the vector. Furthermore, together with the relation $\left[L_{0}, W_{n}^{i}\right]=-n W_{n}^{i}$ (1.7), property (H3) implies that every vector in $\mathcal{H}$ is annihilated by all $W_{n}^{i}$ with sufficiently large $n$, and (because $L_{1}$ augments the $L_{0}$-eigenvalue by 1 ) that any vector $v \in \mathcal{H}$ satisfies $\left(L_{1}\right)^{N} v=0$ for large enough $N$.

Let me now analyse the structure of $\mathcal{W}$ a bit further. Because of its graded structure (see equation (1.8)), as a vector space $\mathcal{W}$ is the direct $\operatorname{sum} \mathcal{W}=\mathcal{W}^{-} \oplus \mathcal{W}^{0} \oplus \mathcal{W}^{+}$of subalgebras $\mathcal{W}^{ \pm}$and $\mathcal{W}^{0}$ which are given by

$$
\mathcal{W}^{ \pm}:=\operatorname{span}\left\{W_{n}^{i} \mid i \in I, \pm n>0\right\}, \quad \mathcal{W}^{0}:=\operatorname{span}\left\{W_{0}^{i} \mid i \in I\right\} .
$$

Further, again because of (11.7) (namely, $\left[L_{0}, W_{n}^{i}\right] \neq 0$ for $n \neq 0$ ), the zero mode subalgebra $\mathcal{W}^{0}$ contains a maximal abelian subalgebra $\mathcal{W}_{\circ} \subset \mathcal{W}$ with $L_{0} \in \mathcal{W}_{\circ}$, and there are subalgebras $\mathcal{W}_{ \pm} \subseteq \mathcal{W}^{0} \oplus \mathcal{W}^{ \pm}$such that

$$
\mathcal{W}=\mathcal{W}_{-} \oplus \mathcal{W}_{\circ} \oplus \mathcal{W}_{+}
$$

is a triangular decomposition of $\mathcal{W}$, which means that

$$
\left[\mathcal{W}_{ \pm}, \mathcal{W}_{\circ} \oplus \mathcal{W}_{ \pm}\right] \subseteq \mathcal{W}_{ \pm}, \quad\left[\mathcal{W}_{+}, \mathcal{W}_{-}\right] \subseteq \mathcal{W}_{\circ}
$$

Lie algebras which enjoy a triangular decomposition possess a distinguished class of modules, the so-called highest weight modules. Such modules $V$ are by definition generated by a highest weight vector $v_{\text {h.w. }}$. This means the following. First,

$$
V \subseteq \mathfrak{U}\left(\mathcal{W}_{-}\right) v_{\text {h.w. }},
$$

i.e. all vectors in a highest weight module $V$ can be obtained from the highest weight vector $v_{\text {h.w. }} \in V$ by acting with the universal enveloping algebra of $\mathcal{W}_{-}$; thus the elements of $\mathcal{W}_{-}$play the rôle of creation operators. Second, all elements of $\mathcal{W}_{+}$act on $v_{\text {h.w. }}$ as annihilation operators:

$$
x_{+} v_{\text {h.w. }}=0 \text { for all } x_{+} \in \mathcal{W}_{+} .
$$


And third, $v_{\text {h.w. }}$ is an eigenvector of the abelian algebra $\mathcal{W}_{\circ}$. In other words, for any highest weight vector $v_{A}$ there is a linear function $\lambda_{A}: \mathcal{W}_{\circ} \rightarrow \mathbb{C}$ such that

$$
x_{\circ} v_{A}=\lambda_{A}\left(x_{\circ}\right) \cdot v_{A}
$$

for all $x_{\circ} \in \mathcal{W}_{\circ}$; I will call $\lambda_{A}$ the weight of $v_{A}$ with respect to $\mathcal{W}_{\circ}$.

Owing to $L_{0} \in \mathcal{W} \circ$ and relation (1.7), every highest weight module of $\mathcal{W}$ satisfies the spectrum condition (H3). However, not any arbitrary highest weight module of $\mathcal{W}$ qualifies as a subspace of $\mathcal{H}$ : the unitarity property (H2) must be satisfied as well. 8 Now every highest weight module $V$ is already endowed with a natural hermitian product; this is uniquely specified by the value on the highest weight vector, say $\left(v_{\text {h.w. }} \mid v_{\text {h.w. }}\right)=1$, namely by choosing a basis of $\mathcal{W}$ in such a way that

$$
\left(W_{n}^{i}\right)^{*}=W_{-n}^{\bar{i}}
$$

where $i \mapsto \bar{i}$ is some involutive permutation of the index set, and then implementing the property (1.14). If the so obtained product is degenerate, then the subspace of $V$ consisting of all null vectors, i.e. vectors $v_{\text {null }}$ which are orthogonal with respect to the form $(\cdot \mid \cdot)$ to all of $V$ (i.e. $\left(v_{\text {null }} \mid w\right)=0$ for all $\left.w \in V\right)$, is a submodule of $V$, and hence $V$ is reducible (but not fully reducible). On the other hand, every unitary highest weight module $V$ is in fact also irreducible, i.e. $\mathcal{W}$ acts on $V$ by an irreducible representation $R$.

Now for any vector $v_{A}$, a simple way to construct a highest weight module is to act freely with the enveloping algebra $\mathfrak{U}\left(\mathcal{W}_{-}\right)$; the module

$$
\mathcal{V}_{A}=\mathfrak{U}\left(\mathcal{W}_{-}\right) v_{A}
$$

obtained by this construction is known as the Verma module generated by $v_{A}$. Verma modules are typically neither irreducible nor unitary; however, in the cases of interest in conformal field theory the submodule of $\mathcal{V}_{A}$ consisting of null vectors is a maximal submodule, and by 'setting the null vectors to zero' (or, in more mathematical terms, by taking the quotient of $\mathcal{V}_{A}$ by this submodule) one arrives at a module $\mathcal{H}_{A}$ which is unitary and irreducible. Also, any highest weight module with highest weight vector $v_{A}$ can be understood as some quotient of $\mathcal{V}_{A}$. It follows that each irreducible sub- $\mathcal{W}$-module of the space $\mathcal{H}$ can be obtained in this fashion, with $A$ some index labelling the module.

The conformal weight of the highest weight state $v_{A}$ of $\mathcal{H}_{A}$ will be denoted by $\Delta_{A}$. When $v \in \mathcal{H}_{A}$ is an eigenvector of $R_{A}\left(L_{0}\right)$ P of eigenvalue $\Delta$, then $m:=\Delta_{A}-\Delta$ is a non-negative integer, which for $v=W_{-m_{1}}^{i_{1}} \cdots W_{-m_{l}}^{i_{l}} v_{A}\left(m_{p} \geq 0\right.$ for $\left.p=1,2, \ldots, l\right)$ can be computed as $m=\sum_{p=1}^{l} m_{p} ; m$ is called the grade of $v$.

\section{Sectors: The Spectrum}

One can summarize some of the results reviewed above by stating that the properties (H1) to (H3) imply that $\mathcal{H}$ has the structure of a (possibly infinite) direct sum

$$
\mathcal{H}=\bigoplus_{A} \mathcal{H}_{A}
$$

\footnotetext{
${ }^{8}$ While unitarity is an obvious requirement in quantum field theory, in statistical mechanics systems it is not always necessary. Nevertheless the $\mathcal{W}$-modules arising in conformal field theory applications to statistical mechanics are highest weight modules (except in so-called 'logarithmic' conformal field theories, which I disregard here, compare section 28), for a reason which is still mysterious to me.

${ }^{9}$ Henceforth, the symbol $R$ will almost always be suppressed.
} 
of unitary irreducible highest weight modules $\mathcal{H}_{A}$ of $\mathcal{W}$. However, these requirements do not specify which of the various unitary irreducible highest weight modules of $\mathcal{W}$ appear in the sum (1.19). The set of irreducible modules which do appear constitutes the spectrum of the conformal field theory; these spaces are also called the physical irreducible modules of $\mathcal{W}$, or the superselection sectors of the theory, or briefly the sectors.

Any observable which appears as a central element of $\mathcal{W}$ not only acts as a constant in each sector $\mathcal{H}_{A}$ (this is just a corollary of Schur's lemma), but in fact it must act by one and the same constant on all sectors. This condition constitutes a strong restriction on the spectrum. Its origin is that central charges cannot be localized; in the context of local observables which are required to form a simple associative algebra, any central charge must therefore be a scalar multiple of the unit operator. Frequently this requirement also arises, at a more practical level, from the fact that one must work with expressions which only make sense when these charges are regarded as numbers; cf. e.g. the Sugawara formula (4.3) below.

It is an important result, known as naturality, that once the eigenvalues of all central elements of $\mathcal{W}$ are fixed (and provided that $\mathcal{W}$ is maximal), in the spectrum of a chiral half of a conformal field theory each (isomorphism class of) unitary irreducible highest weight module appears precisely once. (The derivation of this assertion [87 is beyond the scope of these lectures.) When the number of sectors is finite, then the conformal field theory is called a rational theory. This name originates from the observation that in a rational conformal field theory both the eigenvalues of (canonically normalized) central elements and all conformal dimensions $\Delta_{A}$ are rational numbers.

Among the spectrum of a conformal field theory there is a distinguished sector $\mathcal{H}_{\circ}$ with $\Delta_{\circ}=0$. Its presence is implied by another basic property of quantum field theory, namely the existence of a vacuum state. This is a vector $v_{\circ}$ in $\mathcal{H}_{\circ}$ (unique up to scalar multiplication) which is invariant under all unbroken symmetries of the theory. In the present context this means that $v_{\circ}$ is in particular $\mathcal{W}_{\circ}$-neutral, i.e. $x_{\circ} v_{\circ}=0$ for all $x_{\circ} \in \mathcal{W}_{\circ}$, or in short, $\lambda_{\circ} \equiv 0$. Naively one might expect that $v_{\circ}$ also has all other symmetries of the theory, in particular that it is conformally invariant, i.e. is annihilated by all $L_{n}$. But for $c \neq 0$ this would be incompatible with the relations (1.3) of $\mathcal{V i r}$. Rather, one can only require that $v_{\circ}$ be annihilated by $L_{n}$ with $n \geq-1$. Similar remarks apply to the action of other elements $W_{n}^{i} \in \mathcal{W}$ on the vacuum; in short, $v_{\circ}$ respects the maximally possible number of symmetries of the theory. As a consequence, $v_{\circ}$ is a highest weight vector of $\mathcal{W}$. The irreducible submodule $\mathcal{H}_{\circ}$ of $\mathcal{H}$ which contains the special vector $v_{\circ}$ is called the vacuum sector of the theory.

That $v_{\circ}$ is annihilated by $L_{n}$ with $n \geq-1$ means that it is both a highest weight vector with respect to $\mathcal{V} \mathfrak{i r}$ and invariant under the subalgebra

$$
\mathcal{P}:=\mathcal{V} \mathfrak{i r}_{0, \pm 1} \equiv \operatorname{span}\left\{L_{1}, L_{0}, L_{-1}\right\} \cong \mathfrak{s l}(2)
$$

of $\mathcal{V i r}$ (the projective, or Möbius, transformations); this subalgebra is isomorphic to $\mathfrak{s l}(2) . \square$ Note that the projective transformations are precisely those whose classical counterpart is welldefined on the whole Riemann sphere so that they can be exponentiated so as to provide finite conformal transformations. Namely, the action of $-z^{n+1} \partial_{z}$ is non-singular at $z=0$ only for $n \geq-1$, and non-singular at $z=\infty$ (where it acts as $w^{1-n} \partial_{w}$ with $w=1 / z$ ) only for $n \leq 1$.

${ }^{10}$ When the field of scalars is restricted from $\mathbb{C}$ to $\mathbb{R}$, one obtains the real form $\mathfrak{s u}(1,1) \cong \mathfrak{s l}(2, \mathbb{R})$ of $\mathfrak{s l}(2)$. Together with the anti-holomorphic counterparts $\bar{L}_{0}, \bar{L}_{ \pm 1}$ one arrives at a real form of $\mathfrak{s o}(4)$; this is precisely the conformal algebra that one would get when 'naively' extrapolating from arbitrary $D>2$ to $D=2$. 
Let me also remark that in some applications one may well need the full conformal invariance, so that according to the remarks above the conformal symmetry must not have an anomaly, i.e. one needs $c=0$. This happens e.g. in string theory. In that case there is an extra contribution to $c$ from the ghost fields which are employed to gauge-fix two-dimensional gravity, so that the requirement is that $c_{\text {matter }}=-c_{\text {ghost }}$, e.g. $c_{\text {matter }}=26$ for the bosonic string. $\square$

For unitarity, the numbers $\Delta_{A}$, and hence the conformal weights of all $L_{0}$-eigenvectors in $\mathcal{H}$, must in fact be non-negative. Namely, consider first vectors $v$ which are quasi-primary, which means that they are annihilated by $L_{1}$. Then due to $\left[L_{1}, L_{-1}\right]=2 L_{0}$ and $\left(L_{-1}\right)^{*}=L_{1}$ one has $\left\|L_{-1} v\right\|^{2}=2 \Delta_{v}\|v\|^{2}$, which by unitarity implies that $\Delta_{v} \geq 0$. (Also, $\Delta_{v}=0$ iff $L_{-1} v=0$, in which case together with $L_{1} v=0$ it follows that $v$ is invariant under the projective subalgebra $\mathcal{P}(1.20)$ of $\mathcal{V i r}$.) Further, for any arbitrary vector $v \in \mathcal{H}$ let $N$ be the smallest natural number such that $\left(L_{1}\right)^{N} v=0$ (which exists, see before equation (1.11)); then the vector $\tilde{v}:=\left(L_{1}\right)^{N-1} v$ is non-zero and also quasi-primary. Since the conformal dimensions of $v$ and $\tilde{v}$ are related by $\Delta_{\tilde{v}}=\Delta_{v}-(N-1), \Delta_{v}<0$ would imply that $\Delta_{\tilde{v}}<0$, which as already discussed cannot happen.

A similar argument shows that $\left\|L_{-n} v_{\circ}\right\|^{2}=\left(n^{3}-n\right) \cdot c / 12$ for all $n \in \mathbb{N}$, so that (taking $n>1$ ) for unitarity it is required that $c>0$. Further constraints on the spectrum are obtained by studying the positivity of $\left\|W_{-n_{1}}^{i_{1}} \cdots W_{-n_{p}}^{i_{p}} v_{A}\right\|$ systematically. That is, at any fixed grade one considers the matrix of inner products of all basis states; a necessary condition for unitarity is that each such matrix has non-negative determinant (one needs not impose strict positivity, because one can 'decouple' null vectors, which corresponds to quotienting the Verma module $\mathcal{V}_{A}$ ). To analyze this condition one writes the inner products as expectation values of $\mathcal{W}$ generators with respect to the highest weight vector, which can be calculated by using the bracket relations of $\mathcal{W}$. In practice this can become very hard; for $\mathcal{W}=\mathcal{V i r}$ the result is that for $c \geq 1$ there is no restriction on the conformal dimensions, while below $c=1$ only the discrete set

$$
c=c_{m}:=1-\frac{6}{m(m+1)} \quad \text { with } m \geq 3
$$

of $c$-values is allowed, and for each of these specific values only a finite set

$$
\Delta=\Delta_{m ; p, q}:=\frac{((m+1) p-m q)^{2}-1}{4 m(m+1)} \quad \text { with } \quad 1 \leq p, q \leq m-1
$$

of conformal weights is possible; the theories with these values of $c$ and $\Delta$ are known as the minimal unitary models of the Virasoro algebra. Thus all unitary theories with $c<1$ are rational. But even when the unitarity requirement is dropped, one still needs $c<1$ for rationality. In other words, for rational theories with central charge $c \geq 1$, the chiral symmetry algebra $\mathcal{W}$ is always larger than $\mathcal{V i r}$.

\section{Conformal Fields}

Even though one is ultimately interested in observable quantities only, i.e. in matrix elements

\footnotetext{
${ }^{11}$ In this context it is less obvious that (the matter part of) the conformal field theory should be unitary. (What one has to achieve is unitarity on a certain cohomology of $\mathcal{V i r}$; for this it is certainly helpful to start from a unitary conformal field theory, but I do not know whether this is really necessary.) Also, typically one 'compactifies' the string theory by stipulating that $c_{\text {matter }}$ is the sum of a contribution from space-time and one from some 'internal' theory. When the space-time is flat, each space-time dimension corresponds to one free boson and hence (see section 12) contributes 1 to $c$; but it is not clear to me why in our world where space-time is only asymptotically flat its contribution to $c$ should have precisely the integral value $c=4$.
} 
of observables, in quantum field theory it is often convenient to employ non-observable objects in intermediate steps of calculations. When such objects are operators on the space $\mathcal{H}$, they are referred to as fields. Generic fields are distinguished from the observables by the fact that in terms of the sector decomposition (1.19) of $\mathcal{H}$, they act as $\mathcal{H}_{A} \rightarrow \mathcal{H}_{B}$ with $B \neq A$, while observables act within each individual subspace $\mathcal{H}_{A}$. 피 In conformal field theory, one realizes field operators $\varphi$ via the so-called state-field correspondence; the basic idea is that one generates all vectors in $\mathcal{H}$ from the vacuum $v_{\circ}$ by applying suitable point-like conformal fields $\varphi(z, \bar{z})$, according to

$$
v_{\Delta, \bar{\Delta}}=\lim _{z, \bar{z} \rightarrow 0} \varphi_{\Delta, \bar{\Delta}}(z, \bar{z}) v_{\circ} .
$$

Here the labels indicate the conformal weights of the fields, respectively of the vectors $v_{\Delta, \bar{\Delta}}$ (usually more labels are needed to distinguish the fields, especially when $\mathcal{W} \supset \mathcal{V i r}$ ). The formula (1.23) is often read as the definition of the vector $v_{\Delta, \bar{\Delta}} \in \mathcal{H}$; in the present spirit, it rather specifies the rôle played by the fields $\varphi$.

The precise meaning of the prescription (1.23) and of the formal objects $\varphi$ introduced there is not at all obvious, and depending on the framework adopted to interpret these quantities it can be a delicate issue to verify that the limit in (1.23) makes sense. To investigate this one best starts with the case where the vector $v_{\Delta, \bar{\Delta}}$ lies in the vacuum sector $\mathcal{H}_{\circ}$, so that the fields are actually observables. Now the maximality requirement imposed on the chiral symmetry algebra $\mathcal{W}$ means in particular that any operator on $\mathcal{H}$ which does not belong to $\mathcal{W}$ changes the sector; thus in order for $v_{\Delta, \bar{\Delta}}$ to lie in $\mathcal{H}_{\circ}$, the fields must be suitable combinations of the generators $W_{n}^{i}$. The correct interpretation of the fields is then as generating functions

$$
W^{i}(z)=\sum_{n \in \mathbb{Z}} z^{-n-\Delta_{i}} W_{n}^{i},
$$

or in other words, the generators of $\mathcal{W}$ are regarded as moments or modes of $W^{i}(z)$. In the case of the Virasoro generators, this generating function

$$
T(z)=\sum_{n \in \mathbb{Z}} z^{-n-2} L_{n},
$$

plays the rôle of the energy-momentum tensor; that is, in models which can also be formulated in a Lagrangian setting, it describes the response of the Lagrangian density to a variation of the space-time metric. 10 One must note that in the formula (1.24) the variable $z$ is introduced just as a formal indeterminate. When $z$ is interpreted in terms of a world sheet, and hence is regarded as a complex variable, then the $W_{n}^{i}$ are just the Fourier-Laurent modes of $W^{i}(z)$, and one must worry about the convergence of expressions like (1.24). One way to attack this problem is to think of fields as operator valued distributions, and to work with localized bounded operators (by smearing with test functions and exponentiating), which can e.g. be analyzed in the framework of Wightman field theory [104] or of the algebraic theory of superselection sectors [70]. Here I rather describe the (much less rigorous) formulation that is based on the work of

\footnotetext{
${ }^{12}$ Such a distinction only makes sense if one demands that the superposition principle is not universally valid. In other words, only those vectors in $\mathcal{H}$ which lie in one of the subspaces $\mathcal{H}_{A}$ are regarded as corresponding to proper physical states.

13 This manipulation makes sense even for theories on flat space-times, because the operations of variation and of taking the limit that the metric becomes flat do not commute.
} 
Belavin, Polyakov and Zamolodchikov [11] and is sometimes called the bootstrap approach. Another alternative, at least as far as the mathematical aspects of the theory, rather than its physical interpretation, are concerned, is to think of $z$ as a formal (in the well-defined mathematical sense) variable; this leads to the concept of a vertex operator algebra 37.

Interpreting $z$ as a complex variable, one can invert the relation (1.24), so as to write the Laurent modes as contour integrals of fields multiplied with powers of $z$,

$$
W_{m}^{i}=\frac{1}{2 \pi \mathrm{i}} \oint_{0} \mathrm{~d} z z^{m+\Delta_{i}-1} W^{i}(z)
$$

(recall that $\Delta_{i} \in \mathbb{Z}$ ), where integration is over some curve encircling zero.

When it comes to genuine fields $\varphi(z, \bar{z})$ rather than observables, it is worth to return to the issue of which properties characterize a 'conformal field theory'. What one has to demand is that the 'space of all fields' carries a representation of the conformal group, respectively of the conformal algebra - analogously to what one is used to from Lorentz covariant theories in which the fields carry an action of the Lorentz group and in which, as a consequence, the excitations can be classified by representations of that group. But not all fields one can think of are on the same footing. In particular, the following ones are distinguished:

- The $(\mathcal{V i r}-)$ primary fields. These are defined by the requirement that the state $v_{\Delta, \bar{\Delta}}(1.23)$ is a $\mathcal{V} \mathfrak{i r}$-highest weight state, i.e. is annihilated by all $L_{n}$ with $n>0$.

- The $(\mathcal{V i r}-)$ quasi-primary fields. For these, $v_{\Delta, \bar{\Delta}}$ is annihilated by $L_{1}$, and hence is a highest weight state of the Möbius subalgebra $\mathcal{P}(1.20)$ of $\mathcal{V} \mathfrak{i r}$.

In the relations of the $\mathfrak{s l}(2)$-algebra $\mathcal{P}$ the central term is absent, so that one can identify the generators $L_{0}, L_{ \pm 1}$ with the corresponding generators of the Witt algebra (1.2). Quasiprimary fields are therefore also called 'non-derivative' fields. In terms of the Laurent modes, the distinguished behavior of these types of fields is demonstrated by (1.6), which in terms of fields can be rewritten as $\left[L_{m}, W^{i}(z)\right]=z^{m+1} \partial W^{i}(z)+(m+1) \Delta_{i} z^{m} W^{i}(z)$ : when (1.6) holds for $m=0$ and \pm 1 , then $W^{i}(z)$ is quasi-primary, while if it holds for all $m \in \mathbb{Z}$, then $W^{i}(z)$ is primary. Analogous relations hold for every (quasi-) primary field $\phi:\left[L_{m}, \phi(z)\right]=z^{m+1} \partial \phi(z)+$ $(m+1) \Delta_{\phi} z^{m} \phi(z)$; in particular, in the limit $z \rightarrow 0$ one obtains

$$
\left[L_{n}, \phi(0)\right]= \begin{cases}0 & \text { for } n>0 \\ \Delta \cdot \phi(0) & \text { for } n=0 \\ \partial \phi(0) & \text { for } n=-1\end{cases}
$$

Non-primary fields are called secondary fields or descendants (and also the term 'ancestor' for primary field is used). Among the fields that correspond to the vectors in an irreducible highest weight module $\mathcal{H}_{A}$ of $\mathcal{W}$ there is precisely one primary field $\phi_{A}$; it corresponds to the highest weight vector. The collection of these fields, consisting of the primary $\phi_{A}$ and all its descendants, is called the family of $\phi_{A}$ and is denoted by $\left[\phi_{A}\right]$. By the grade of a descendant of $\phi_{A}$ one means the grade of the corresponding vector in $\mathcal{H}_{A}$.

The simplest example of a primary field, present in every conformal field theory, is the identity field $\mathbf{1}$. It satisfies $\partial \mathbf{1}=0$, and hence corresponds to the vacuum vector $v_{\circ}$; its first non-trivial descendant is the energy-momentum tensor $T(z)$ :

$$
\lim _{z \rightarrow 0} T(z) v_{\circ}=\sum_{n \in \mathbb{Z}} z^{-n-2} L_{n} v_{\circ}=L_{-2} v_{\circ}
$$


$T(z)$ is the prime example of a quasi-primary field.

The Lie brackets of the Laurent components of all quasi-primary fields in the chiral symmetry algebra $\mathcal{W}$ read $\left[W_{m}^{i}, W_{n}^{j}\right]=d_{i j} p^{i} \delta_{m+n, 0}+\sum_{k} C_{k}^{i j} q_{m n}^{i j k} W_{m+n}^{k}$, where the structure constants $p^{i}$ (which are combinations of eigenvalues of central charges) and $q_{m n}^{i j k}$ are rational numbers for which explicit expressions in terms of $\Delta_{i}$, respectively, $m, n$ and $\Delta_{i}, \Delta_{j}, \Delta_{k}$ are known [14]. Here I use the notations $d^{i j}:=\left\langle W_{\Delta_{i}}^{i} W_{-\Delta_{j}}^{j}\right\rangle$ and $C^{i j k}:=\left\langle W_{\Delta_{i}}^{i} W_{-\Delta_{j}}^{j} W_{\Delta_{j}-\Delta_{i}}^{k}\right\rangle$ as well as $\sum_{j} d^{i j} d_{j k}=\delta_{k}^{i}$ and $C_{k}^{i j}:=\sum_{l} C^{i j l} d_{l k}$, where $\langle\cdots\rangle$ denotes the vacuum expectation value, defined as

$$
\langle X\rangle:=\left(v_{\circ} \mid X v_{\circ}\right) .
$$

Bounded operators on a Hilbert space can be multiplied. Analogously one would like to multiply in a suitable way the fields $\varphi(z, \bar{z})$ that occur in the present setting. Just like in other approaches to quantum field theory there arises the problem that such products tend to be singular, at least in the limit of 'coinciding points'. A way out is to consider normal ordered expressions, i.e. set

$$
: W_{m}^{i} W_{n}^{j}:= \begin{cases}W_{m}^{i} W_{n}^{j} & \text { for } n>0 \\ W_{n}^{j} W_{m}^{i} & \text { for } n \leq 0\end{cases}
$$

(say) for bilinears in the modes $W_{n}^{i}$. (The normal ordering prescription adopted here is not at all the only possible one. Whenever one changes the treatment of only a finite number of terms (in a quite arbitrary manner), one obtains another sensible normal ordering.) The normal ordered products (1.30) share the property of the generators $W_{n}^{i}$ to act locally nilpotently on $\mathcal{H}$. Also, the normal ordering of fields $W^{i}(z)$ is obtained from the normal ordering of their modes $W_{n}^{i}$ via the series expansion (1.24). It follows e.g. that all commutators of normal ordered products of fields in $\mathcal{W}$ are fully determined by those of the energy-momentum tensor and of the Virasoro-primary fields in $\mathcal{W}$, and that normal ordered products of quasi-primary fields are generically not quasi-primary.

\section{The Operator Product Algebra}

In a chiral conformal field theory - a chiral half of a two-dimensional theory - the fields depend on a single complex variable $z$. As long as one deals with a single field, say $W^{i}(z)$, it suffices to describe it for values of $z$ which lie on a closed curve encircling zero, say the unit circle, since then one recovers all its modes $W_{n}^{i}$ by the formula (1.26). However, as soon as one intends to analyze products of fields, one must at least 'smear' the circle. Namely, when expressed in terms of fields, the abstract Lie bracket of modes should correspond to the commutator with respect to a suitable associative product; now inserting (1.26) into a Lie bracket yields (with a suitable labelling of integration variables) the difference

$$
\left[W_{m}^{i}, W_{n}^{j}\right]=\left[\frac{1}{2 \pi \mathrm{i}} \oint_{0} \mathrm{~d} z \frac{1}{2 \pi \mathrm{i}} \oint_{0} \mathrm{~d} w-\frac{1}{2 \pi \mathrm{i}} \oint_{0} \mathrm{~d} w \frac{1}{2 \pi \mathrm{i}} \oint_{0} \mathrm{~d} z\right] z^{m+\Delta_{i}-1} w^{n+\Delta_{j}-1} W^{i}(z) W^{j}(w)
$$

of two double contour integrals, where in each term the integrations must be performed in the indicated order. One would like to rewrite this expression in a form where there is a single contour integration over fields $W^{k}(z)$ multiplied with suitable powers of the position, so as to recover the result for the Lie bracket $\left[W_{m}^{i}, W_{n}^{j}\right]$ as a linear combination of modes $W_{p}^{k}$. To achieve this, one interprets the first pair of integrations as to be performed for $|z|>|w|$ and the 
second for $|w|>|z|$. Thus the formal product of fields appearing in (1.31) is to be understood as the radially ordered product $\Re$, defined for arbitrary fields $\varphi_{1}$ and $\varphi_{2}$ by

$$
\Re\left(\varphi_{1}(z) \varphi_{2}(w)\right):= \begin{cases}\varphi_{1}(z) \varphi_{2}(w) & \text { for }|z|>|w| \\ \varphi_{2}(w) \varphi_{1}(z) & \text { for }|w|>|z| .\end{cases}
$$

For fixed value of $w$ (say) one can now deform the two $z$-contours in the formula (1.31) and merge them to a single one encircling the point $w$. One then arrives at the desired result for the commutator by postulating that the radially ordered product of two fields whose positions are sufficiently close can be written as a linear combination of fields evaluated at (say $\left.{ }^{14}\right) w$, multiplied with suitable powers of $z-w$. The so obtained decomposition is called the operator product expansion of the fields $W^{i}(z)$ and $W^{j}(w)$.

When one knows the structure constants of $\mathcal{W}$, one can determine the singular part of operator product expansions by requiring that the contour integrations $\oint_{0} \mathrm{~d} w \oint_{w} \mathrm{~d} z$ yield the correct result. E.g. the Virasoro algebra amounts to the product

$$
\Re(T(z) T(w))=\frac{1 / 2}{(z-w)^{4}} C+\frac{2}{(z-w)^{2}} T(w)+\frac{1}{z-w} \partial_{w} T(w)+\mathcal{O}_{\text {reg }} .
$$

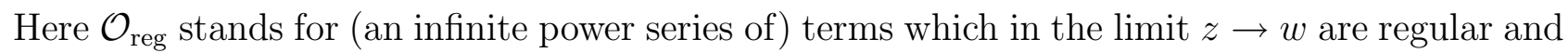
hence do not affect the result for $\left[L_{m}, L_{n}\right]$. (More generally, only singular terms in expansions like (1.33) are relevant to the observables; the regular terms in fact do not have any meaning independent of the sector, e.g. their precise form depends on the choice of normal ordering prescription in the different sectors.) It is common to refer to radially ordered products (1.32) just as operator products and to omit the symbol $\Re$; I will usually follow this habit.

In the two-dimensional theory, we have both the chiral and the antichiral algebras $\mathcal{W}$ and $\overline{\mathcal{W}}$; they commute, and correspondingly the operator product of fields $W^{i}(z)$ and $\bar{W}^{j}(\bar{w})$ just coincides with their normal ordered product. But we may also consider operator products of the $W^{i}(z)$ with arbitrary fields $\varphi$. For instance, for the operator product of a primary field with $T$ one finds

$$
T(z) \phi(w, \bar{w})=\frac{\Delta}{(z-w)^{2}} \phi(w, \bar{w})+\frac{1}{z-w} \partial_{w} \phi(w, \bar{w})+\mathcal{O}_{\text {reg }}
$$

which upon Laurent expansion correctly reproduces the formula (1.27) for the commutator $\left[L_{n}, \phi\right]$.

The singular terms in an operator product $\varphi_{1}(z) \varphi_{2}(w)$ of the type above are called the contraction of $\varphi_{1}$ and $\varphi_{2}$ and are denoted by $\varphi_{1}(z) \varphi_{2}(w)$. Also, the term of order $(z-w)^{0}$ is essentially the normal ordered product $: \varphi_{1} \varphi_{2}:-$ when the particular normal ordering prescription

$$
: \varphi_{1}(z) \varphi_{2}(z)::=\frac{1}{2 \pi \mathrm{i}} \oint_{w} \mathrm{~d} z(w-z)^{-1} \Re\left(\varphi_{1}(z) \varphi_{2}(w)\right)
$$

(which is slightly different from the one presented in (1.30)) is adopted, then it is indeed precisely the normal ordered product, i.e. one has

$$
\varphi_{1}(z) \varphi_{2}(w)=\varphi_{1}(z) \varphi_{2}(w)+: \varphi_{1}(z) \varphi_{2}(z):+O(z-w)
$$

\footnotetext{
${ }^{14}$ One may also choose the positions of these fields at $z$, or also at $(z+w) / 2$ or $\sqrt{w z}$ or any other function which smoothly approaches $w$ in the limit $z \rightarrow w$.
} 
Besides the conformal invariance, a second basic property of two-dimensional conformal field theories is that operator products cannot only be defined when one of the fields belongs to the chiral algebra, but for arbitrary pairs of fields. That is, there is a closed operator product algebra among all fields, and the operation involved is indeed a product in the sense that it is associative. It is expected that this structure is a direct consequence of fundamental properties of quantum field theory. But when trying to construct it this way one faces quite a few subtleties, and to the best of my knowledge a complete derivation from first principles has never been given. Instead, one usually postulates the existence of a closed associative operator product algebra as a separate input, called the bootstrap hypothesis or requirement. Note, however, that operator product expansions do not converge in the operator norm, but only weakly, i.e. when applied to a vector in the state space $\mathcal{H}$. Thus while one may regard the collection of all fields as a vector space (over some function field), the operator product does not really make this space into an associative algebra in the mathematical sense. ${ }^{\text {to }}$

Operator products of primary fields $\phi$ can be written in the form

$$
\phi_{A}(z, \bar{z}) \phi_{B}(w, \bar{w})=\sum_{C} \mathcal{C}_{A B} C(z-w)^{\Delta_{C}-\Delta_{A}-\Delta_{B}}(\bar{z}-\bar{w})^{\bar{\Delta}_{C}-\bar{\Delta}_{A}-\bar{\Delta}_{B}} \phi_{C}(w, \bar{w})+\ldots,
$$

where the ellipsis stands for contributions of descendant fields $\varphi_{C} \in\left[\phi_{C}\right]$. The numbers $\mathcal{C}_{A B} C$ introduced in (1.37) are called the operator product coefficients of the theory. The corresponding coefficients which involve descendants are completely fixed by the $\mathcal{W}$-symmetry in terms of those of the primaries (and of their conformal weights and the values of central charges). For instance, the coefficient with which the descendant $\partial^{n} \varphi$ of a quasi-primary field $\varphi$ appears equals the coefficient for $\varphi$ multiplied by $(n !)^{-1} \prod_{l=0}^{n-1}\left(l+\Delta_{A}-\Delta_{B}+\Delta_{\varphi}\right) /\left(l+2 \Delta_{\varphi}\right)$. On the other hand, the relation between the operator product coefficients for quasi-primaries and those for primaries has been worked out in some detail only for $\mathcal{W}=\mathcal{V i r}$ [11. Nevertheless, at least in principle it is possible to solve the theory completely, i.e. to compute all correlation functions, by expressing them in terms of the coefficients $\mathcal{C}_{A B}{ }^{C}$ involving only primaries and of the structure constants of $\mathcal{W}$. The determination of the numbers $\mathcal{C}_{A B}{ }^{C}$ is therefore one of the main goals in conformal field theory.

\section{Correlation Functions and Chiral Blocks}

Operator product expansions like (1.34) or (1.37) can only be valid when applied to vectors in $\mathcal{H}$. In fact, because the presence of additional fields typically affects manipulations with integration contours, strictly speaking they even only hold when matrix elements with respect

\footnotetext{
15 The theory of vertex operator algebras [37] provides a means for formulating operator products in a purely algebraic setting, without having to resort to arguments based on complex analysis, e.g. contour deformation or analytic continuation. The basic structures of a vertex operator algebra are an infinite set of products and the action of the derivative $\partial$; the commutator with respect to the first of these products yields the Lie algebra structure of the space $\mathcal{W}_{\circ}$ of zero modes. The variables $z$ etc. are treated as formal variables, which implies e.g. that $(z-w)^{-1}$ is interpreted as $z^{-1}(1-w / z)^{-1}=z^{-1} \sum_{n \geq 0}(w / z)^{n}$. Thus in particular $1 /(z-w)+1 /(w-z)=z^{-1} \sum_{n \in \mathbb{Z}}(w / z)^{n}$, which in terms of complex analysis corresponds to the delta function on the unit circle. The relation with the radial ordering prescription is that upon re-interpreting the variables as complex numbers, the formal power series occurring in the vertex operator formulation become convergent series precisely if the relevant numbers are radially ordered in the appropriate manner.
} 
to vectors in $\mathcal{H}$ are taken, e.g. for vacuum matrix elements

$$
\begin{aligned}
\mathcal{G}\left(\left\{z_{j}\right\},\left\{\bar{z}_{j}\right\}\right) & \equiv\left\langle\varphi_{1}\left(z_{1}, \bar{z}_{1}\right) \varphi_{2}\left(z_{2}, \bar{z}_{2}\right) \cdots \varphi_{p}\left(z_{p}, \bar{z}_{p}\right)\right\rangle \\
& :=\left(v_{\circ} \mid \varphi_{1}\left(z_{1}, \bar{z}_{1}\right) \varphi_{2}\left(z_{2}, \bar{z}_{2}\right) \cdots \varphi_{p}\left(z_{p}, \bar{z}_{p}\right) v_{\circ}\right) .
\end{aligned}
$$

Such a vacuum expectation value is called the ( $p$-point) correlation function of the fields $\varphi_{1}, \varphi_{2}, \ldots, \varphi_{p}$.

Given the operator product algebra, one can in principle evaluate any correlation function by expressing successively all products as linear combinations of fields until one ends up with a linear combination of fields acting on $v_{\mathrm{o}}$, and then using the fact (see below) that the only field with non-vanishing one-point function is the identity primary field $\mathbf{1}$. In practice, this procedure only works in simple cases such as free field theories. However, some general properties of correlators can be derived without a detailed knowledge of the operator products. They are implied by the so-called Ward identities for $\mathcal{W}$, which are obtained as follows. Consider a correlation function of the form $\left\langle W^{i}(z) \varphi_{1}\left(z_{1}, \bar{z}_{1}\right) \varphi_{2}\left(z_{2}, \bar{z}_{2}\right) \cdots\right\rangle$, multiplied with some power $z^{n}$, and perform a $z$-integration over a contour $\mathcal{C}$ that encircles all of the 'insertion points' $z_{i}$. By deforming $\mathcal{C}$ to infinity one learns that for $n>-\Delta_{i}$ this integrated correlator vanishes; on the other hand, after deforming the contour into a union of contours each of which encircles precisely one of the insertion points $z_{j}$, one can insert the operator product $W^{i}(z) \varphi_{1}\left(z_{j}, \bar{z}_{j}\right)$ and perform the integration for each of these contours separately. These manipulations amount to a linear differential equation for the correlation function $\left\langle\varphi_{1}\left(z_{1}, \bar{z}_{1}\right) \varphi_{2}\left(z_{1}, \bar{z}_{1}\right) \cdots\right\rangle$. In particular, in the case of $W^{i}(z)=T(z)$, the operator product (1.34) leads to the projective Ward identities

$$
\sum_{i=1}^{p} z_{i}^{n}\left(z_{i} \frac{\partial}{\partial z_{i}}+(n+1) \Delta_{i}\right) \mathcal{G}\left(\left\{z_{j}\right\},\left\{\bar{z}_{j}\right\}\right)=0 \quad \text { for } n \in\{0, \pm 1\}
$$

for correlation functions of primary fields. When $\mathcal{W}=\mathcal{V i r}{ }^{10}$ and the number $p$ of insertions is small, the projective Ward identities are particularly effective:

- The relation (1.39) with $n=-1$ tells us that one-point functions are constant. Thus they satisfy $\left\langle\phi_{A}\right\rangle=\lim _{z \rightarrow 0}\left\langle\phi_{A}(z)\right\rangle=\left(v_{\circ} \mid v_{A}\right)$, which is non-zero only if $\phi_{A}=\phi_{\circ}=\mathbf{1}$.

- In the case of two-point functions $\mathcal{G} \equiv \mathcal{G}_{A B}=\left\langle\phi_{A}\left(z_{1}\right) \phi_{B}\left(z_{2}\right)\right\rangle$, the general solution of the $n=-1$ identity is $\mathcal{G}=\mathcal{G}\left(z_{1}-z_{2}\right)$; the $n=0$ identity then gives $\mathcal{G}_{A B} \propto\left(z_{1}-z_{2}\right)^{-\Delta_{A}-\Delta_{B}}$, and finally $n=1$ shows that $\Delta_{A}=\Delta_{B}$. When the chiral symmetry algebra $\mathcal{W}$ is maximally extended, the constant of proportionality (which is not fixed by the Ward identities, since they are linear), is zero unless $\phi_{2}$ is the conjugate field of $\phi_{1}$, which carries the complex conjugate representation of $\mathcal{W}$. Thus $\left\langle\phi_{A} \phi_{B}\right\rangle \propto \delta_{A, B^{+}}$, where $\phi_{A^{+}}=\left(\phi_{A}\right)^{+}$.

- Similarly, three-point functions are completely fixed up to a multiplicative constant:

$$
\mathcal{G}_{A B C} \propto\left(z_{1}-z_{2}\right)^{\Delta_{C}-\Delta_{A}-\Delta_{B}}\left(z_{2}-z_{3}\right)^{\Delta_{A}-\Delta_{B}-\Delta_{C}}\left(z_{3}-z_{1}\right)^{\Delta_{B}-\Delta_{C}-\Delta_{A}} .
$$

- For four-point functions the situation is already more involved (they will be studied in more detail in section 14 and section 24). The projective Ward identities only imply that every four-point function can be written as an arbitrary function of a certain variable $w$, multiplied with definite powers (which are linear combinations of the conformal weights) of the coordinate differences $z_{i}-z_{j}$. This variable $w$ is a cross-ratio of the insertion points; e.g. one may choose

\footnotetext{
${ }^{16}$ When $\mathcal{W}$ is strictly larger than $\mathcal{V} \mathfrak{i r}$, a more detailed analysis is required.
} 
$w=\left(z_{2}-z_{1}\right)\left(z_{3}-z_{4}\right) /\left(z_{3}-z_{1}\right)\left(z_{2}-z_{4}\right)$, which is obtained by applying to all insertion points the (finite) projective transformation $z \mapsto\left(z_{2}-z_{1}\right)\left(z-z_{4}\right) /\left(z-z_{1}\right)\left(z_{2}-z_{4}\right)$, upon which $z_{1}, z_{2}, z_{3}, z_{4}$ are mapped to $\infty, 1, w, 0$. In other words, without loss of generality we can set three of the insertion points to $\infty, 1,0$, i.e. consider correlators of the form

$$
\mathcal{G}(z, \bar{z}) \equiv \mathcal{G}_{A B C D}(z, \bar{z})=\left\langle\phi_{A}(\infty, \infty) \phi_{B}(1,1) \phi_{C}(z, \bar{z}) \phi_{D}(0,0)\right\rangle
$$

(In the case of three-point functions one can analogously map the insertion points to the fixed values $\infty, 1,0$, which explains why in that case the dependence on the $z_{i}$ is completely fixed. More generally, any $p$-point function with $p \geq 3$ can be written as a function of $p-3$ cross-ratios, multiplied by powers of the coordinate differences $z_{i}-z_{j}$.)

The Ward identities involve only one chiral half of the theory, so that when studying them one can ignore the presence of the antichiral half. Of course, there are analogous identities involving the antichiral algebra, too. As a consequence, the correlation function of a two-dimensional theory that is obtained by combining its two chiral halves can be expressed as a linear combination of the products of independent solutions $\mathcal{F}_{I}(z)$ and $\overline{\mathcal{F}}_{I}(\bar{z})$ of the chiral and antichiral Ward identities,

$$
\mathcal{G}(z, \bar{z})=\sum_{I=1}^{M} \sum_{\bar{I}=1}^{\bar{M}} a_{I \bar{I}} \mathcal{F}_{I}(z) \overline{\mathcal{F}}_{I}(\bar{z}) .
$$

The individual solutions $\mathcal{F}_{I}$ and $\overline{\mathcal{F}}_{I}$ are called the chiral blocks (or also the conformal blocks) of $\mathcal{G}$.

For $p>3$ the sum (1.42) contains, in general, more then one term (see also section 14), and hence does not factorize into a chiral and an antichiral part. This means in particular that (except for the observables $W^{i}(z)$ ) such a factorization is not possible for the fields $\varphi=\varphi(z, \bar{z})$. $\square$ But still, as also indicated by the form (1.42) of correlators, one can regard primary fields $\phi$ as combinations

$$
\phi_{A}(z, \bar{z})=\sum_{q} c_{q} \varpi_{q}(z) \bar{\varpi}_{\bar{q}}(\bar{z})
$$

of suitable chiral objects $\varpi_{q}$ and $\bar{\varpi}_{\bar{q}}$, which are called chiral vertex operators. In order for this decomposition to make sense, for each primary $\phi_{A}$ the label $q$ must be understood as standing not only for $A$, but also for two additional primary field labels $B, C \square$ such that $\varpi_{q} \equiv \varpi_{A ; B, C}$ constitutes a map $\mathcal{H}_{B} \rightarrow \mathcal{H}_{C}$ which intertwines the action of the chiral algebra, i.e. $\varpi_{A ; B, C} \circ R_{B}\left(W_{n}^{i}\right)=R_{C}\left(W_{n}^{i}\right) \circ \varpi_{A ; B, C}$. In terms of operator products, the restriction to a specific source $\mathcal{H}_{B}$ and range $\mathcal{H}_{C}$ corresponds to considering only the terms involving the family $\left[\phi_{C}\right]$ in the operator product $\phi_{A} \phi_{B}$; correspondingly, the coefficients $c_{q} \equiv c_{A ; B, C}$ in $(1.43)$ are in fact nothing but the operator product coefficients $\mathcal{C}_{A B} C$.

By construction, the chiral blocks can be interpreted as the vacuum expectation values of suitable chiral vertex operators, e.g. $\mathcal{F}_{I}=\left\langle\varpi_{\circ ; A, A} \varpi_{A ; B, I} \varpi_{I ; C, D^{+}} \varpi_{D^{+} ; D, \circ}\right\rangle$ for the four-point function $\mathcal{G}_{A B C D}$. Chiral blocks are generically not functions, but are multi-valued. They are single-valued on a suitable multiple covering of the complex plane (and hence can be regarded

\footnotetext{
17 Note in particular that the notation $\varphi(z)$ that I occasionally used above was just an abbreviation to indicate the $z$-dependence of $\varphi(z, \bar{z})$.

18 as well as possibly some multiplicity label, compare section 9.

19 Sometimes the blocks are also called holomorphic respectively anti-holomorphic blocks. Thus in this context the term 'holomorphic' is used in a rather loose sense; even the full correlation functions are in general only meromorphic (they can have a pole whenever two insertion points coincide).
} 
as sections of some (projectively flat) bundle over the Riemann sphere). Analytic continuation of a chiral block connects the different sheets of that covering. Correspondingly, the exchange of two chiral vertex operators is governed by a representation of the braid group rather than the permutation group.

The requirement that the chiral and antichiral blocks combine to single-valued correlation functions of the two-dimensional theory yields algebraic equations for the linear coefficients $a_{I \bar{I}}$ in (1.42). In principle, these equations can be solved to obtain these coefficients up to over-all normalization; the latter is left undetermined because the Ward identities are linear differential equations. For the three-point functions there is often only a single chiral block and hence only a single coefficient $a \equiv a_{A B C}$. In this case comparison with the operator product algebra (1.37) shows that $a_{A B C}=\mathcal{C}_{A B C}:=\sum_{D} \mathcal{C}_{A B}{ }^{D} d_{D C}$, where

$$
d_{A B}:=\left(z_{1}-z_{2}\right)^{2 \Delta_{A}}\left(\bar{z}_{1}-\bar{z}_{2}\right)^{2 \bar{\Delta}_{A}}\left\langle\phi_{A}\left(z_{1}\right) \phi_{B}\left(z_{2}\right)\right\rangle \propto \delta_{A, B^{+}}
$$

plays the rôle of a metric in the space of fields. In short, the three-point functions of primary fields are essentially the operator product coefficients.

\section{Lecture 2: $\quad$ Fusion Rules, Duality and Modular Transformations}

\section{Fusion Rules}

In the first lecture we learned that upon forming radially ordered products and when considered inside correlation functions, the fields $\varphi$ of a conformal field theory realize a closed associative operator product algebra. Unfortunately, in practice this structure looks extremely complicated. However, a large amount of information about the operator products is already contained in a much more transparent structure, the so-called fusion rules. Roughly speaking, the fusion rules constitute the basis-independent contents of the operator product algebra, i.e. count the number $\mathrm{N}_{A B}^{C}$ of times that the family $\left[\phi_{C}\right]$ of the primary field $\phi_{C}$ appears in the operator product of primaries $\phi_{A}$ and $\phi_{B}$. In other words, they tell how many distinct couplings among primary fields, respectively $\mathcal{W}$-families, are possible. To encode this information, one associates to each primary field $\phi_{A}$ an abstract object $\Phi_{A}$, and introduces an abstract multiplication ' $\star$ ' by writing

$$
\Phi_{A} \star \Phi_{B}=\sum_{C} \mathrm{~N}_{A B}^{C} \Phi_{C} .
$$

The integers $\mathrm{N}_{A B}^{C}$ are known as the fusion rule coefficients. Note that the product (2.1) is not isomorphic to the ordinary tensor product of modules of the chiral symmetry algebra $\mathcal{W} .20$

Let me comment on how to actually read the fusion rules off the operator product algebra. The $\mathcal{W}$-family $\left[\phi_{C}\right]$ occurs in the product of $\phi_{A}$ with $\phi_{B}$ iff $\mathrm{N}_{A B}{ }^{C}$ is non-zero. This might suggest

20 This follows directly from the observation that upon forming tensor products all central charges add up (e.g. $c_{\text {tot }}=c_{1}+c_{2}$ ), whereas the fusion product yields an object which appears in the same theory and hence has the same values of central charges as the original fields. (For a description of fusion products which closely resembles a ( $z$-dependent) tensor product, see [59]. Another approach to fusion products [107] is via Connes fusion.)

${ }^{21}$ In practice, one rather proceeds the other way round, i.e. given the fusion rules, one determines (though not uniquely) differential equations for chiral blocks, and thereby the operator product coefficients, from general principles of conformal field theory. For some details see section 14. 
that $\mathrm{N}_{A B}^{C}$ can take only the values 0 or 1 , corresponding to the alternative whether $\left[\phi_{C}\right]$ appears in $\phi_{A}(z) \phi_{B}(w)$ or not. But one and the same family $\left[\phi_{C}\right]$ may couple in several distinct ways to $\phi_{A}$ and $\phi_{B}$ so that values $\mathrm{N}_{A B}{ }^{C}>1$ are possible as well. Accordingly, the fields $\varphi_{C}$ appearing in the expansion (1.37) are not necessarily all distinct, i.e. it may happen that $\varphi_{C_{1}}=\varphi_{C_{2}}$ for $C_{1} \neq C_{2}$; nevertheless it is not allowed just to add up the corresponding operator product coefficients, because the relative values of coefficients involving different members of a family $\left[\phi_{k}\right]$ are fixed by the Ward identities of $\mathcal{W}$. If the full operator product algebra is known, the presence of a coefficient $\mathrm{N}_{A B}^{C}$ larger than unity can therefore be inferred as follows. One subtracts the leading contribution to the operator product $\phi_{A} \phi_{B}$, corresponding to the descendant $\varphi$ of $\phi_{C}$ with the lowest allowed grade, and likewise all contributions involving descendants at higher grades whose presence is dictated by the Ward identities; then $\mathrm{N}_{A B}^{C}>1$ iff afterwards there is still some contribution to $\phi_{A} \phi_{B}$ left over which involves members of $\left[\phi_{C}\right]$. It follows e.g. that for $\mathrm{N}_{A B}^{C}>1$ the minimal grades of the fields that contribute to the $\mathrm{N}_{A B}{ }^{C}$ different couplings between $\phi_{A}, \phi_{B}$ and $\left[\phi_{C}\right]$ must all be distinct. (E.g. there is at most one non-vanishing coupling among the primaries $\phi_{A}, \phi_{B}, \phi_{C}$; this is actually a necessary condition for excluding chiral blocks with logarithmic singularities.)

It turns out to be convenient to formalize the general properties of fusion rules which are implied by the principles of conformal field theory that I outlined in lecture 1 . To this end one regards the objects $\Phi_{A}$ and numbers $\mathrm{N}_{A B}{ }^{C}$ as the basis elements and structure constants of a ring over the integers $\mathbb{Z}$ or of an algebra over the complex numbers $\mathbb{C}$. These structures are called the fusion ring, respectively the fusion algebra, of the conformal field theory; their defining properties are the following:

(F1) they are commutative and associative, and they have a unit element (namely, $\Phi_{\circ}$, the abstract object associated to the identity primary field);

(F2) there is a distinguished basis (namely the one consisting of the $\Phi_{A}$ ) in which the structure constants are non-negative and which contains the unit element;

(F3) the evaluation at the unit element provides an involutive automorphism, called the conjugation and denoted by $\Phi_{A} \mapsto\left(\Phi_{A}\right)^{+}$.

In terms of the structure constants $\mathrm{N}_{A B}{ }^{C}$, these properties read as follows.

(N1) $\mathrm{N}_{B A}^{C}=\mathrm{N}_{A B}^{C}, \quad \sum_{D} \mathrm{~N}_{A B}^{D} \mathrm{~N}_{D C}^{E}=\sum_{D} \mathrm{~N}_{B C}^{D} \mathrm{~N}_{D A}^{E}$ and $\mathrm{N}_{\circ B}^{C}=\delta_{B}^{C}$;

(N2) $\mathrm{N}_{B A}{ }^{C} \geq 0$;

(N3) $C_{A B} \equiv \mathrm{N}_{A B}^{\circ}=\delta_{A, B^{+}}$for some order-two permutation $A \mapsto A^{+}$of the index set (such that $\left.\left(\Phi_{A}\right)^{+}=\Phi_{A^{+}}\right)$, and $\left(A^{+}\right)^{+}=A$ as well as $\mathrm{N}_{A^{+}{ }_{B^{+}}}^{C^{+}}=\mathrm{N}_{A B}{ }^{C}$.

It then also follows that $\mathrm{N}_{A B C}:=\mathrm{N}_{A B} C^{+}$is totally symmetric.

Note that in the identification of the $\Phi_{A}$ as a basis it is implicit that for all $A, B$ the number $\sum_{C} \mathrm{~N}_{A B}^{C}$ is finite; conformal field theories which satisfy this requirement are called quasi-rational. A rational conformal field theory, i.e. one with only a finite number of sectors, is also quasi-rational; while this is not manifest in the definition, it follows easily from the very existence of a fusion ring. It is worth stressing that (quasi-) rationality is not a fundamental property of conformal field theories. However, in practice it is often indispensable, since it allows one to perform many calculations explicitly. This manifests itself for the first time when one studies the representation theory of the fusion ring. (Whether the concept of a fusion ring is still applicable in non-quasi-rational theories is not known.) Accordingly, I will from now on restrict my attention to rational conformal field theories only, unless stated otherwise.

The fusion ring of a rational conformal field theory is a finite-dimensional commutative 
associative ring. As a consequence, each of its irreducible representations is one-dimensional, and every finite-dimensional representation is isomorphic to the direct sum of such irreducible representations. In particular, the adjoint representation $\pi_{\mathrm{ad}}$, defined by $\pi_{\mathrm{ad}}\left(\Phi_{A}\right):=\mathrm{N}_{A}$, where $\mathrm{N}_{A}$ denotes the matrix with entries $\left(\mathrm{N}_{A}\right)_{B}{ }^{C}=\mathrm{N}_{A B}{ }^{C}$, must be isomorphic to the direct sum of one-dimensional irreducible representations (in fact, each inequivalent one-dimensional representation appears in this sum precisely once). In other words, there exists a unitary matrix $S$ which 'diagonalizes the fusion rules' in the sense that - simultaneously for all values of the label $A$ - the matrix

$$
\mathrm{D}_{A}:=S^{-1} \mathrm{~N}_{A} S
$$

is diagonal, and also the relation $C S=S^{*}$ is valid.

It should be noted that the row and column labels of the matrix $S$ that is introduced this way are a priori on a rather distinct footing: the row index labels the elements $\Phi_{A}$ of the distinguished basis, while the column index counts the inequivalent one-dimensional irreducible representations $\pi_{B}$. While the two sets of labels have the same order (so that, as I already did above, one can use the same symbols for either type of labels), in general there does not exist a canonical bijection between them. As it turns out, however, for those fusion rings which occur in conformal field theory such a canonical bijection does exist, and moreover, when implementing this bijection the diagonalizing matrix $S$ possesses the highly non-trivial property of being symmetric, and also satisfies $S_{A \circ}>0$ (in particular $S_{A \circ} \in \mathbb{R}$ ) for all values of the label $A$. It then follows that the one-dimensional irreducible representations $\pi_{A}$ obey

$$
\pi_{A}\left(\Phi_{B}\right) \equiv\left(\mathrm{D}_{B}\right)_{A}^{A}=S_{B A} / S_{\circ A}
$$

As a consequence, the relation (2.2) amounts to an expression

$$
\mathrm{N}_{A B}^{C}=\sum_{D} \frac{S_{A D} S_{B D}\left(S^{-1}\right)_{C D}}{S_{\circ D}}
$$

of the fusion rule coefficients in terms of the matrix $S$, and also that $S^{2}=C$, so that (2.4) can be rewritten more symmetrically as $\mathrm{N}_{A B C}=\sum_{D} S_{A D} S_{B D} S_{C D} / S_{\circ D}$.

\section{Duality}

The associativity of the operator product algebra implies that the fusion rules are associative as well. I will now use the same information to deduce properties of the correlation functions. Let us study the four-point functions $\mathcal{G}=\left\langle\phi_{A} \phi_{B} \phi_{C} \phi_{D}\right\rangle$ of primary fields. Applying the operator product algebra on the first two and on the last two fields, one has $\left\langle\phi_{A} \phi_{B} \phi_{C} \phi_{D}\right\rangle=$ $\left\langle\left(\phi_{A} \phi_{B}\right)\left(\phi_{C} \phi_{D}\right)\right\rangle \equiv\left\langle\Re\left(\Re\left(\phi_{A} \phi_{B}\right) \Re\left(\phi_{C} \phi_{D}\right)\right)\right\rangle$, which pictorially amounts to

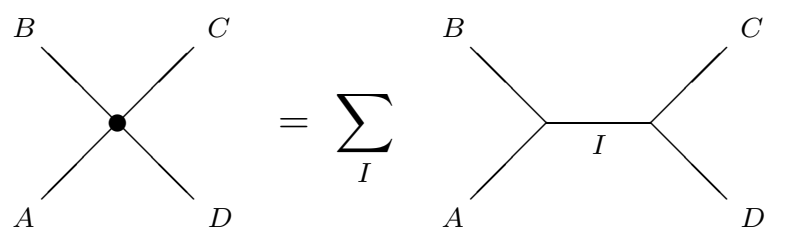

${ }^{22}$ This is not yet the Verlinde 105] formula, because at this point there is no connection to modular transformations yet - this interpretation must be postponed until section 11 . 
But using associativity of the operator product algebra, one could also form products in a different order (in fact this is implicit in the very notation: usually I do not write any brackets to indicate the order in which multiple products are performed), resulting in pictures like

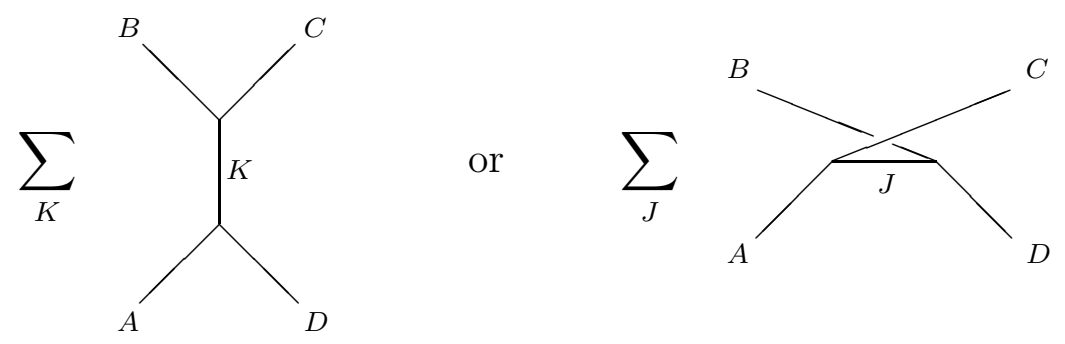

Of course, because of the radial ordering prescription each of these choices is only valid for a definite order of the absolute values of the insertion points $z_{i}$ of the fields $\phi_{A}, \ldots, \phi_{D}$, and hence the different pictures describe functions which coincide only upon an appropriate analytic continuation. Now when the two chiral halves of a two-dimensional theory are combined, so that $\bar{z}_{i}$ is identified as the complex conjugate of $z_{i}$, one must require that every correlation function is single-valued on the Riemann sphere when the dependence on both $z_{j}$ and $\bar{z}_{j}$ is accounted for; thus the three descriptions above for the four-point correlators $\mathcal{G}$ must yield one and the same function. Together with (1.42) this yields the crossing symmetry relations for the four-point functions, which when choosing the insertion points as in formula (1.41) read

$$
\mathcal{G}_{A B C D}(z, \bar{z})=\mathcal{G}_{B C D A}(1-z, 1-\bar{z})=z^{-2 \Delta_{C}} \bar{z}^{-2 \bar{\Delta}_{C}} \mathcal{G}_{A C B D}\left(z^{-1}, \bar{z}^{-1}\right) .
$$

On the other hand, each of the pictures describes the chiral blocks in a different 'channel', and hence combining the two chiral halves amounts to three different decompositions of the four-point function as a sum over products of chiral blocks (for more details, see section 14 below). In a quasi-rational theory the sums are finite, and as a consequence [84] the different systems of chiral blocks are linearly related:

$$
\begin{aligned}
& \hat{\mathcal{F}}_{A B C D, K}\left(z_{1}, z_{2}, z_{3}, z_{4}\right)=\sum_{I} \mathrm{~F}_{K I}\left[\begin{array}{l}
B C \\
A D
\end{array}\right] \mathcal{F}_{A B C D, I}\left(z_{2}, z_{3}, z_{4}, z_{1}\right), \\
& \check{\mathcal{F}}_{A B C D, J}\left(z_{1}, z_{2}, z_{3}, z_{4}\right)=\sum_{I} \mathrm{~B}_{J I}\left[\begin{array}{l}
B C \\
A D
\end{array}\right] \mathcal{F}_{A B C D, I}\left(z_{1}, z_{3}, z_{2}, z_{4}\right) .
\end{aligned}
$$

The coefficients $\mathrm{F}\left[\begin{array}{l}B C \\ A D\end{array}\right]$ and $\mathrm{B}\left[\begin{array}{c}B C \\ A D\end{array}\right]$ that appear in these linear relations are called the fusing and braiding matrices, and the corresponding manipulations with chiral blocks as visualized in the pictures above are referred to as fusing and braiding transformations, or generically as duality transformations. Strictly speaking, the indices $I$ and $J$ that I used to label the various blocks in a given channel generically do not just count the intermediate primary fields, but for the case of fusion rule coefficients larger than 1 also the various possible couplings among their families, i.e. they stand for multi-indices, e.g. $I \equiv(\alpha, I, \beta)$ with $\alpha \in\left\{1,2, \ldots, \mathrm{N}_{A B}{ }^{I}\right\}, \beta \in\left\{1,2, \ldots, \mathrm{N}_{I C}{ }^{D}\right\}$. For notational simplicity, in (2.8) and also below I suppress all multiplicity indices.

The fusing and braiding matrices obey a number of compatibility relations, which can be derived by considering suitable duality transformations of higher $p$-point functions and

23 Those readers who want to see them are referred to e.g. 44 .

The relation with the notation used there is $\mathrm{F}_{\alpha I \beta, \gamma J \delta}[\stackrel{A B}{C D}]=F_{\alpha I \beta, \gamma J \delta}^{(A B C)_{D}}$ and

$\mathrm{B}_{\alpha I \beta, \gamma J \delta}\left[\begin{array}{l}A B \\ C D\end{array}\right]=\sum_{K} \sum_{\lambda, \mu, \nu}\left(\left(F^{(B A C)_{D}}\right)^{-1}\right)_{\alpha I \beta, \lambda K \nu} R_{\lambda, \mu}^{(A B)_{K}} F_{\mu K \nu, \gamma J \delta}^{(A B C)_{D}}$. 
demanding that the result does not depend on the individual set of transformations, but only on the initial and final configurations of (external and intermediate) fields. These relations can all be reduced to those obtained for five-point functions and which involve five and six different configurations, respectively 88; the latter are called the polynomial equations, or more specifically, the pentagon and hexagon equations and read

$$
\begin{aligned}
& \mathrm{F}_{H I}\left[\begin{array}{c}
A B \\
E F
\end{array}\right] \mathrm{F}_{J F}\left[\begin{array}{l}
C D \\
E G
\end{array}\right]=\sum_{K} \mathrm{~F}_{K F}\left[\begin{array}{l}
B D \\
I G
\end{array}\right] \mathrm{F}_{J I}\left[\begin{array}{c}
A K \\
E G
\end{array}\right] \mathrm{F}_{H K}\left[\begin{array}{l}
A B \\
J D
\end{array}\right], \\
& \sum_{K} \mathrm{~B}_{K I}\left[\begin{array}{l}
A B \\
D E
\end{array}\right] \mathrm{B}_{G E}\left[\begin{array}{l}
A C \\
K F
\end{array}\right] \mathrm{B}_{H K}\left[\begin{array}{c}
B C \\
D G
\end{array}\right]=\sum_{L} \mathrm{~B}_{L E}\left[\begin{array}{c}
B C \\
I F
\end{array}\right] \mathrm{B}_{H I}\left[\begin{array}{l}
A C \\
D L
\end{array}\right] \mathrm{B}_{G L}\left[\begin{array}{l}
A B \\
H F
\end{array}\right]
\end{aligned}
$$

(note that the required summations do not amount to matrix multiplication of the $\mathrm{F}$ and $\mathrm{B}$ matrices). When also correlation functions on higher genus Riemann surfaces are considered, then there arises one additional independent relation, obtained from manipulating one-point blocks on the torus. In principle one may start from the fusion rules to set up the system (2.9) of polynomial equations and then solve this system for the $F$ and $B$ matrices, but already for a small number of sectors this becomes very hard (see [44] for examples).

\section{Counting States: Characters}

The structure of an irreducible highest weight module $\mathcal{H}_{A}$ of the chiral algebra $\mathcal{W}$ is rather complicated. But a lot of information is already contained in its weight system, i.e. in the collection of weights $\lambda$ of the vectors in a basis of $\mathcal{H}_{A}$. To keep track of the weight system of a module, one sums over formal exponentials $\mathrm{e}^{\lambda}$ of its weights, counting their multiplicities, or what is equivalent (writing $\mathrm{e}^{\lambda}=\left(v_{\lambda} \mid \mathrm{e}^{W_{\circ}} v_{\lambda}\right)$ with $v_{\lambda}$ the orthonormal basis vectors of $\mathcal{H}_{A}$ ), takes the trace over the module of formal exponentials of elements $W_{\circ}$ of $\mathcal{W}_{\circ}$. The so obtained quantity

$$
\chi_{A}\left(W_{\circ}\right):=\operatorname{tr}_{\mathcal{H}_{A}} \mathrm{e}^{W_{\circ}}
$$

is called the character of the irreducible $\mathcal{W}$-module $\mathcal{H}_{A}$. (Compare the characters of irreducible modules of Lie algebras, see section 20).

It is often convenient to regard $\chi_{A}$ as a function of complex variables, which are the coefficients in an expansion of a generic element $W_{\circ}$ of $\mathcal{W}_{\circ}$ in some suitable basis, and for some purposes it is sufficient to specialize to characters at vanishing values of some of these variables. A very specific, but nevertheless most important, case is provided by the Virasoro-specialized character, for which $W_{\circ}$ is taken to have only a component in the direction of $L_{0}-\frac{1}{24} C$ (the inclusion of the central term is for simpler modular transformation properties):

$$
\chi_{A}(\tau):=\operatorname{tr}_{\mathcal{H}_{A}} \mathrm{e}^{2 \pi \mathrm{i} \tau\left(L_{0}-C / 24\right)} .
$$

It is a function of one complex variable $\tau$ and (provided that the chiral algebra $\mathcal{W}$ is of 'at most polynomial growth', which seems to be the case in all conformal field theories) converges

${ }^{24}$ That this construction makes sense has its origin in the fact that for any set of numbers $a_{n}$ indexed by $\mathbb{Z}$, the formal Laurent series $f(q):=\sum_{n} a_{n} q^{n}$ in some indeterminate $q$ contains the same information as the $a_{n}$. Here this recipe is generalized to the case where multiplicities are labelled by weights rather than by integers; accordingly one associates to each weight $\lambda$ a formal variable $\mathrm{e}^{\lambda}$ on which one imposes the usual properties of exponentials, i.e. $\mathrm{e}^{\lambda} \mathrm{e}^{\mu}=\mathrm{e}^{\lambda+\mu}$ and $\mathrm{e}^{0}=1$. In more mathematical terms, the basic observation is that the weights form an abelian group $L$ under vector addition. The formal exponentials constitute a basis for the group algebra $\mathbb{C} L$ of $L$. When considered as elements of $\mathbb{C} L$, one can add up these exponentials, and hence can consider $\chi_{A}$ as an element of this group algebra, respectively, in the case of infinite-dimensional modules, of some completion of the group algebra. 
in the upper half-plane. By construction, $\chi_{A}(\tau) \cdot \exp \left(-2 \pi \mathrm{i} \tau\left(\Delta_{A}-c / 24\right)\right)$ is a power series in $q=\exp (2 \pi \mathrm{i} \tau)$, with the coefficient of $q^{n}$ the dimension of the subspace of vectors of grade $n$.

The $\mathcal{V i r}$-specialized character $\chi_{A}(\tau)$ can be interpreted as a chiral block for the '0-point correlation function' (or vacuum-to-vacuum amplitude) on a torus with modular parameter $\tau$, which in the diagrammatic description used in (2.5) corresponds to a one-loop graph without any external lines. By identifying $L_{0}-C / 24$ as a Hamiltonian, $\chi_{A}(\tau)$ can also be regarded as a partition function,

$$
\chi_{A}(\tau)=\operatorname{tr}_{\mathcal{H}_{A}} \mathrm{e}^{-\beta H},
$$

in a thermal state of complex inverse temperature $\beta=-2 \pi \mathrm{i} \tau$; in particular, $\tau \rightarrow \mathrm{i} 0$ and $\tau \rightarrow \mathrm{i} \infty$ correspond to the high and low temperature limit, respectively.

Once the characters are known, the computation of the 0-point function on the torus for the full two-dimensional conformal field theory with symmetry algebra $\mathcal{W} \oplus \overline{\mathcal{W}}$ amounts to specifying non-negative integers $Z_{A B}$ which tell how often the chiral block corresponding to the irreducible $\mathcal{W}$-representation labelled by $A$ gets combined with the antichiral block for the irreducible $\overline{\mathcal{W}}$-representation labelled by $B$. The total partition function of the conformal field theory is then given by

$$
\left.\mathcal{Z} \equiv \mathcal{Z}(\tau, \bar{\tau})\right|_{\bar{\tau}^{*}=\tau}:=\left.\sum_{A, B} \chi_{A}(\tau) Z_{A B} \chi_{B}(\bar{\tau})\right|_{\bar{\tau}^{*}=\tau}
$$

where we have to identify $\bar{\tau}$ with the complex conjugate of $\tau$. To qualify as a partition function of a physical theory, the matrix $Z$ clearly must fulfill a number of consistency requirements. By construction, its entries $Z_{A B}$ are non-negative integers; moreover, the vacuum sector $\mathcal{H}_{\circ}$ must be unique, and hence one needs $Z_{00}=1$. Further properties arise from modular invariance, to be discussed in the next section.

\section{Modularity}

The space of characters of the unitary irreducible highest weight modules of the chiral algebra $\mathcal{W}$ of a rational conformal field theory carries a unitary representation of the group $\mathrm{SL}_{2}(\mathbb{Z})$. (The elements of $\mathrm{SL}_{2}(\mathbb{Z})$ are $2 \times 2$-matrices with integral entries and determinant one.) To show this crucial fact of life would go much beyond the scope of this review, and in fact I am not aware of any rigorous proof. 0 A matrix $M \in \mathrm{SL}_{2}(\mathbb{Z})$ acts on the characters by a change of parameters which depends in a specific way on the entries of $M$; in the particular case of Virasoro-specialized characters, the action is given by

$$
\tau \mapsto M \tau:=\frac{a \tau+b}{c \tau+d} \quad \text { for } \quad M=\left(\begin{array}{cc}
a & b \\
c & d
\end{array}\right)
$$

$(a, b, c, d \in \mathbb{Z}, a d-b c=1)$, i.e. $M \cdot \chi(\tau):=\chi(M \tau)$. Note that here the elements $M$ and $-M$ of $\mathrm{SL}_{2}(\mathbb{Z})$ act in the same way, so that the Virasoro-specialized characters actually carry a representation of the quotient group $\operatorname{PSL}_{2}(\mathbb{Z})=\mathrm{SL}_{2}(\mathbb{Z}) /\{\mathbb{1},-\mathbb{1}\}$. This group is known as the modular group of the torus, and correspondingly the mapping (2.14) is called a modular transformation.

The parameter $\tau$ which (for convergence of $\chi(\tau)$ ) takes values in the upper complex halfplane can indeed be interpreted geometrically as parametrizing a torus, namely the one obtained

\footnotetext{
25 Actually in all known theories the $\mathrm{SL}_{2}(\mathbb{Z})$-representation factorizes through some finite-index subgroup.
} 
by identifying the opposite edges of the quadrangle with corners $0,1, \tau$ and $1+\tau$ in the complex plane. The complex structure on this torus depends on $\tau$ only up to modular transformations.

The group $\mathrm{SL}_{2}(\mathbb{Z})$ is freely generated by two elements $S$ and $T$ modulo the relations $S^{2}=(S T)^{3}$ and $S^{4}=\mathbb{1}$. For $\operatorname{PSL}_{2}(\mathbb{Z})$ these get supplemented by the relation $S^{2}=\mathbb{1}$; in the general case, $S^{2}=C$ is the matrix for the conjugation $A \mapsto A^{+}$, i.e. $C_{A B}=\delta_{A, B^{+}}$; thus the action of modular transformations on the space of characters of primary fields is a genuine representation of the twofold cover $\mathrm{SL}_{2}(\mathbb{Z})$ of the modular group $\mathrm{PSL}_{2}(\mathbb{Z})$ precisely when the conjugation is non-trivial. The elements $S$ and $T$ are represented on the modular parameter $\tau$ as

$$
T: \tau \mapsto \tau+1 \quad \text { and } \quad S: \tau \mapsto-1 / \tau .
$$

(In terms of the temperature $\beta^{-1}=(-2 \pi \mathrm{i} \tau)^{-1}, S$ exchanges the low and high temperature regimes - or, thinking of $\beta$ as a coupling constant, the strong and weak coupling regions.) The corresponding matrices that act on the space of characters as $\chi_{A}(\tau+1)=\sum_{B} T_{A B} \chi_{B}(\tau)$ and $\chi_{A}(-1 / \tau)=\sum_{B} S_{A B} \chi_{B}(\tau)$ are, correspondingly, referred to as the (modular) S-matrix and T-matrix of the conformal field theory. From the definition (2.11) of Virasoro-specialized characters it follows immediately that the T-matrix is diagonal and unitary, with entries

$$
T_{A A}=\exp \left(2 \pi \mathrm{i} \Delta_{A}-c / 24\right) .
$$

In contrast, the explicit form of the S-matrix, which turns out to be a symmetric and unitary matrix, is much more difficult to obtain; in fact it is by no means obvious that the modular inversion $S$ closes on the space of characters. In particular, knowing only the Virasoro-specialized characters is typically not sufficient to determine $S$ (e.g., conjugate fields $\phi_{A}$ and $\phi_{A^{+}}$have identical Virasoro-specialized characters); rather one must use the full characters.

It is by no means accidental that in the definition (2.2) I used the same letter $S$ for the matrix that diagonalizes the fusion rules as for the matrix that implements $\tau \mapsto-1 / \tau$. Namely, for a modular invariant rational conformal field theory this diagonalizing matrix (when normalizations are chosen in such a way that it is symmetric) coincides with the modular inversion $S$ as introduced here. With this identification, the identity (2.4) expressing the fusion rule coefficients through $S$ is known as the Verlinde formula 105.20

In order to construct a conformal field theory consistently on a Riemann surface of genus larger than zero, the partition function (2.13) must be invariant under the modular transformations of that surface, in particular under (2.14) in the case of the torus which has genus 1. I should point out that, as a consequence, modular invariance is a basic property of any conformal field theory model that is relevant to string theory, but that it is not a fundamental property of conformal field theory as such, and there are situations where it is absent. (For instance, in the case of the critical Ising model modular invariance restricts the number of sectors to three, which have the same highest weight with respect to the chiral and the antichiral Virasoro algebra. These correspond to the identity field, the energy operator and the order parameter of the Ising model, as listed in table (4.20) below. But for many purposes one must also consider other sectors, which correspond to the disorder parameter and to a chiral

${ }^{26}$ The existing derivations of the Verlinde formula range from formal manipulations with chiral blocks (see e.g. exercise 3.6 in [88]), localization of path integrals [12] and surgery manipulations on three-manifolds [4] to highly non-trivial arguments in algebraic geometry (10, 29]; for reviews and further references, see [9] and [102]). Typically the more rigorous the arguments are, the more restricted is the range of theories to which they apply; e.g. the algebraic geometry derivation applies only to the case of (most) WZW theories. 
or antichiral free fermion.) Also, in general more requirements than just modular invariance are necessary for the existence and consistency of a conformal field theory. It is therefore not surprising that there exist modular invariant partition functions which cannot correspond to any conformal field theory (for some examples, namely so-called extensions of WZW theories by spin-1 currents which do not describe a conformal embedding, see 46]).

The requirement that (2.13) is invariant under the modular transformations (2.14) is equivalent to demanding that the matrix $Z=\left(Z_{A B}\right)$ satisfies

$$
[Z, S]=[Z, T]=0
$$

i.e. commutes with both the $\mathrm{S}$ - and the T-matrix. An immediate consequence of $[Z, T]=0$ is that the 'conformal spin', i.e. the combination $\Delta-\bar{\Delta}$ of conformal dimensions, must be an integer, so that each field is 'local' with respect to all other fields. As a consequence, at any genus all correlation functions of the two-dimensional theory are single-valued functions.

There always exists a straightforward solution to the constraints (2.17), namely $Z=\mathbb{1}$. This is called the diagonal or A-type modular invariant. When $\mathcal{W}$ is maximal, then this is in fact 87] the only solution, up to possibly a permutation which is an automorphism of the fusion rules, i.e. $Z_{A B}=\delta_{A, \pi B}$ with $\mathrm{N}_{\pi, \pi B}^{\pi_{C} C}=\mathrm{N}_{A B}^{C}$. However, often one does not know the maximal chiral algebra, so that it makes sense to analyze the constraint (2.17) also in the case of non-maximal $\mathcal{W}$ (but with the theory still being rational). As it turns out, 2.17) constitutes a powerful restriction, and its implementation is a highly non-trivial task. So far the solutions have been classified only for very specific types of theories, e.g. for the WZW theories (see section 21) based on $A_{1}^{(1)}$, the Virasoro minimal models [21], for WZW theories based on $A_{2}^{(1)}$ [62], and for $N=2$ superconformal minimal models 64 .

\section{Free Bosons}

The simplest examples of conformal field theories are those which describe massless free bosons or fermions. Here I will sketch the bosonic case. The classical action for a massless free boson $X$ living on a $D$-dimensional space-time manifold of metric $g_{\mu \nu}$ reads $\mathrm{S}_{X} \propto \int \mathrm{d}^{D} x \sqrt{\operatorname{det} g} g^{\mu \nu} \partial_{\mu} X \partial_{\nu} X$. Variation of $\mathrm{S}_{X}$ with respect to $g_{\mu \nu}$ yields the energy-momentum tensor

$$
T_{\mu \nu}=-\partial_{\mu} X \partial_{\nu} X+\frac{1}{2} g_{\mu \nu} \sum_{\sigma, \tau} g^{\sigma \tau} \partial_{\sigma} X \partial_{\tau} X
$$

which is conserved $\left(\sum_{\mu} \partial^{\mu} T_{\mu \nu}=0\right)$ and has trace $\sum_{\mu} T_{\mu}^{\mu} \propto 1-D / 2$. Thus in $D=2$ dimensions $T_{\mu \nu}$ is traceless; in complex coordinates, this means $T_{z \bar{z}}=0$, so that conservation reduces to $\partial_{\bar{z}} T_{z z}=0=\partial_{z} T_{\bar{z} \bar{z}}$, or in other words, $T \equiv T_{z z}=T(z)$ and $\bar{T} \equiv T_{\bar{z} \bar{z}}=\bar{T}(\bar{z})$, as is needed for a conformal field theory.

As the energy-momentum tensor $T$ has conformal weight $\Delta=2$, at the classical level the boson $X$ has scaling dimension zero. Correspondingly, $X$ is not a proper conformal field in the sense of section 5 ; it can be written as $X(z, \bar{z})=X(z)+\bar{X}(\bar{z})$ where $X(z)$ has an expansion

$$
X(z)=X_{0}-\mathrm{i} P \ln (z)+\mathrm{i} \sum_{n \in \mathbb{Z} \backslash\{0\}} \frac{1}{n} J_{n} z^{-n} .
$$

Thus $X$ is not single-valued on the complex plane. But the derivatives $\partial X$ and $\bar{\partial} \bar{X}$ (which appear in the action $\mathrm{S}_{X}$ ) are, and so are (suitably normal ordered) exponentials $: \mathrm{e}^{\mathrm{i} q X}$ :. The 
canonical commutation relations for the free boson $X$ yield Heisenberg relations for its modes: $\left[X_{0}, J_{n}\right]=0$ for $n \neq 0$ and

$$
\left[X_{0}, P\right]=\mathrm{i}, \quad\left[J_{m}, J_{n}\right]=m \delta_{m+n, 0} .
$$

The chiral algebra $\mathcal{W}$ of this theory is the semi-direct sum of the Virasoro algebra generated by $T(z)$ and a Heisenberg algebra which is generated by the Virasoro-primary field $J=\sum_{n \in \mathbb{Z}} J_{n} z^{-n-1}\left(J_{0} \equiv P\right)$. In terms of $X$ these fields read

$$
J(z)=\mathrm{i} \partial X(z), \quad T(z)=-\frac{1}{2}: \partial X(z) \partial X(z):=\frac{1}{2}: J^{2}(z):
$$

Using the two-point function $\langle X(z) X(w)\rangle \simeq-\ln (z-w)$ and Wick's rule for calculating correlators of normal ordered products of free fields, one checks that the conformal central charge has the value $c=1$ and that $J$ has conformal weight $\Delta_{J}=1$. The zero mode subalgebra $\mathcal{W}_{\circ}$ is spanned by $L_{0}$ and the zero mode $J_{0} \equiv P$ of the field $J$. The primary fields of this theory are $\phi_{q}=: \mathrm{e}^{\mathrm{i} q X}:$; they are labelled by charges, i.e. $J_{0}$-eigenvalues, $q \in \mathbb{R}$, and their fusion rules read $\Phi_{p} \star \Phi_{q}=\Phi_{p+q}$.

To be precise, these statements refer to the situation that the modes of the classical boson $X$, in particular the zero mode $X_{0}$, are allowed to take values on the whole real line $\mathbb{R}$. In contrast, when the boson is compactified on a circle of radius $R$, in the sense that one identifies the values $X_{0}$ and $X_{0}+2 \pi R$ of the zero mode, and when in addition $R^{2}=2 \mathcal{N}$ is an even integer, then there are two additional (Virasoro- and $J$-primary) fields in $\mathcal{W}$ which have conformal dimension $\Delta=\mathcal{N}$. Moreover, in this case the theory is rational, and with a suitable normalization of the current $J$ the possible charge values are $q \in\{0,1, \ldots, 2 \mathcal{N}-1\}$. The conformal dimension of a primary field of charge $q$ is then $\Delta_{q}=q^{2} / 4 \mathcal{N}$. The fusion rules read $\Phi_{p} \star \Phi_{q}=\Phi_{p+q \bmod 2 \mathcal{N}}$, and the modular S-matrix has entries

$$
S_{p q}=\exp (-\pi \mathrm{i} p q / \mathcal{N}) / \sqrt{2 \mathcal{N}}
$$

Similarly, when $R^{2}=2 r / s(r, s$ coprime) is any other rational number, the theory is still rational and the chiral algebra $\mathcal{W}$ contains additional fields of conformal weight $\Delta=\mathcal{N}:=r s$ in $\mathcal{W}$. But in the general case $\mathcal{W}$ contains fields of still higher weight, and one deals with a non-diagonal extension of the theory that has squared radius $2 \mathcal{N}$. However, the theories with radius $R$ and radius $2 / R$, which look most different when formulated in terms of a classical action, are actually one and the same conformal field theory; in particular for $R^{2}=2 / \mathcal{N}$ one recovers a diagonal theory.

All the theories just described have $c=1$. In fact, any other unitary rational $c=1$ conformal field theory can be formulated in terms of a free boson as well. Namely, each of those theories can be obtained as a so-called orbifold of the theory of a free boson on a circle. In general, forming an orbifold of a conformal field theory means that one restricts the observables to those invariant under some (discrete) group Z of automorphisms. In the case at hand, Z must be a discrete subgroup of $\mathrm{O}(3)$, which leads to three isolated rational theories for which $\mathrm{Z}$ is non-abelian, and to a continuous family of theories for which $\mathrm{Z}=\mathbb{Z}_{2}$, with the non-trivial element corresponding to the identification of $X$ with $-X$. For rational square radius, the latter $\mathbb{Z}_{2}$-orbifolds are again rational theories, but for non-rational values of $R^{2}$ they are not even quasi-rational.

More generally, free bosons are important not only as theories in their own right, but can serve as a starting point for the description of many other theories, too. A particularly 
interesting possibility is to modify the theory in such a way that $X$ still has the correlation functions of a free boson, but that the form (2.21) of $T(z)$ is changed the by addition of a term proportional to $\partial^{2} X$, which corresponds to the presence of a background charge in a Coulomb gas. This way one can in particular obtain a free field realization of the minimal series (1.21) of unitary models with $c<1$.

\section{Simple Currents}

Each fusion ring contains a distinguished basis element, the unit element $\Phi_{\circ}$. This element clearly has the property to be invertible within the ring, i.e. in mathematical terms, it is a unit of the ring. But a fusion ring may contain also further basis elements which are units in this sense. These are called simple currents of the ring, or of the conformal field theory, and they turn out to be of considerable importance. In the theory of a free boson at rational square radius that was just described, in fact each basis element $\Phi_{p}$ is a simple current, with inverse $\left(\Phi_{p}\right)^{-1}=\Phi_{2 \mathcal{N}-p}$. Another important example for a simple current is the field which implements the GSO projection in superstring theory. In this section I sketch the most intriguing properties of simple currents; for more details see e.g. [100] and [43].

A simple current $J$ can be equivalently characterized as a primary field for which the fusion product with the conjugate field just yields the unit element,

$$
\mathrm{J} \star \mathrm{J}^{+}=\Phi_{\circ}
$$

or for which the fusion rules are simple in the sense that for each $A$ the fusion product $\mathrm{J} \star \Phi_{A}$ belongs again to the distinguished basis (it is then simply written as $\Phi_{\mathrm{J} A} \equiv \Phi_{\mathrm{J} \star A}$ ). In terms of the fusion rule coefficients this means that $\sum_{C} \mathrm{~N}_{J B}{ }^{C}=1$ for all $B$. Yet another characterization is that $S_{\circ \mathrm{J}}=S_{\circ \circ}$ (while $S_{\circ A}>S_{\circ \circ}$ for every $\Phi_{A}$ which is not a simple current). ${ }^{27}$ Due to the associativity of the fusion product, the product of two simple currents is again a simple current. Simple currents thus form an abelian group under the fusion product, i.e. a subgroup of the fusion ring (this group has been termed the center of the theory). A rational theory can of course accommodate only finitely many simple currents. This implies that simple currents are unipotent; the smallest positive integer $N \equiv N_{\mathrm{J}}$ such that $\mathrm{J}^{N}=\Phi_{\circ}$ is called the order of $\mathrm{J}$ (here $\mathrm{J}^{2} \equiv \mathrm{J} \star \mathrm{J}$, etc.). Furthermore, any simple current organizes the primary fields into orbits $\left[\Phi_{A}\right]:=\left\{\Phi_{A}, \Phi_{\mathrm{J} A}, \Phi_{\mathrm{J}^{2} A}, \ldots, \Phi_{\mathrm{J}^{N-1} A}\right\}$; the size $N_{A}:=\left|\left[\Phi_{A}\right]\right|$ of any orbit $\left[\Phi_{A}\right]$ is a divisor of the order $N=\left|\left[\Phi_{\circ}\right]\right|$ of $\mathrm{J}$.

A crucial result about simple currents [100, 75] is that in a unitary theory, S-matrix elements involving fields on the same simple current orbit differ only by a phase:

$$
S_{\mathrm{J}^{p} A, \mathrm{~J}^{q} B}=\mathrm{e}^{2 \pi \mathrm{i} p \mathrm{Q}_{\mathrm{J}}(B)} \mathrm{e}^{2 \pi \mathrm{i} q \mathrm{Q}_{\mathrm{J}}(A)} \mathrm{e}^{2 \pi \mathrm{i} p q \mathrm{Q}_{\mathrm{J}}(\mathrm{J})} \cdot S_{A B} .
$$

Here $\mathrm{Q}_{\mathrm{J}}(A)$ is the monodromy charge of $\phi_{A}$ with respect to $\mathrm{J}$, defined as the combination

$$
\mathrm{Q}_{\mathrm{J}}(A):=\Delta_{\mathrm{J}}+\Delta_{A}-\Delta_{\mathrm{J} A} \bmod \mathbb{Z}
$$

27 The term 'current' is chosen [99] in anticipation of the fact that these fields can be used for an extension of the chiral symmetry algebra, and (also when the conformal dimension is not equal to 1) such a field is often called a current. The qualification 'simple' refers to the behavior with respect to fusion rules (and to correlation functions as well, see 45]), but also fits with the fact that in algebraic quantum field theory a superselection sector with statistical dimension equal to 1 is called a simple sector. 
of conformal weights (in a rational theory, $\mathrm{Q}_{\mathrm{J}}(A)$ is a rational number). The monodromy charge is additive under the operator product, i.e. for any $C$ with $\mathrm{N}_{A B}^{C} \neq 0$ one has $\mathrm{Q}_{\mathrm{J}}(A)=$ $\mathrm{Q}_{\mathrm{J}}(B)+\mathrm{Q}_{\mathrm{J}}(C) \bmod \mathbb{Z}$. Based on (2.24), one can show that for any simple current which satisfies $N_{\mathrm{J}} \Delta_{\mathrm{J}} \in \mathbb{Z}$, the matrix $Z$ with entries

$$
Z_{A B}=\frac{N}{N_{A}} \sum_{n=0}^{N_{A}-1} \delta_{B, \mathrm{~J}^{n} A} \delta^{(\mathbb{Z})}\left(Q_{\mathrm{J}}(A)+\frac{n}{2} Q_{\mathrm{J}}(\mathrm{J})\right)
$$

provides a modular invariant. Here $\delta^{(\mathbb{Z})}$ denotes the function with $\delta^{(\mathbb{Z})}(x)=1$ for $x \in \mathbb{Z}$ and $\delta^{(\mathbb{Z})}(x)=0$ otherwise. Note that all non-zero entries of $Z$ are between fields on the same simple current orbit. If the center is the cyclic group $\left\{\Phi_{\circ}, \mathrm{J}, \mathrm{J}^{2}, \ldots, \mathrm{J}^{N-1}\right\} \cong \mathbb{Z}_{N}$ generated by a simple current J, then (2.26) is the only modular invariant of the theory with this property. When the center is not cyclic, then there are further possibilities for simple current extensions which are parametrized by the so-called discrete torsion [82].

When $\Delta_{\mathrm{J}}$ is integral, the presence of $\delta^{(\mathbb{Z})}$ in (2.26) means that only fields with vanishing monodromy charge occur, and the modular invariant reduces to

$$
\mathcal{Z}=\sum_{A: Q_{\mathrm{J}}(A)=0} \frac{N}{N_{A}}\left|\sum_{n=0}^{N_{A}-1} \chi_{\mathrm{J}^{n} A}\right|^{2} .
$$

Invariants of this type are called integer spin simple current extensions. They are interpreted as diagonal invariants of a theory with an extended chiral algebra $\mathcal{W}$, with the additional fields in $\mathcal{W}$ being the simple currents $\mathrm{J}^{n}$. Note that typically several irreducible modules of the original chiral algebra $\mathcal{W}_{\text {unext. }}$ combine to a single irreducible module of $\mathcal{W}$; according to (2.27), the characters of the irreducible $\mathcal{W}$-modules are proportional to the orbit sum $\sum_{n} \chi_{\mathrm{J}}{ }^{n}$. Moreover,

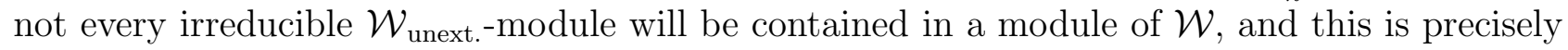
encoded in the requirement of vanishing monodromy charge.

The orbit sums of characters of the original 'unextended' theory appear in (2.27) with multiplicities $N / N_{A}$. Orbits $A$ with $N / N_{A}>1$ are called fixed points of the simple current J. To interpret these multiplicities, one must recall (see section 11 ) that for maximally extended $\mathcal{W}$ each (equivalence class of) irreducible $\mathcal{W}$-module appears precisely once. Thus a multiplicity in front of the complete square indicates that there exist several non-isomorphic $\mathcal{W}$-modules which reduce to one and the same module of $\mathcal{W}_{\text {unext. }}$, or in other words, that such a term corresponds to several distinct primary fields in the conformal field theory with the enlarged chiral algebra. However, this prescription is unambiguous only for $N / N_{A}=2$ or 3 . For larger values it can also happen that one must include (part of) the prefactor $N / N_{A}$ into the extended character; as this can only be done for complete squares, the number of distinct possible interpretations is then equal to the number of ways that $N / N_{A}$ can be written as a sum of squares. For example, when $N / N_{A}=5$ one either deals with five inequivalent $\mathcal{W}$-modules which have ( $\mathcal{W}_{\text {unext.-specialized })}$ character $\sum_{n=0}^{N_{A}-1} \chi_{\mathrm{J}^{n} A}$, or else with one such module plus another $\mathcal{W}$-module whose character equals twice that sum.

Given an invariant of the type (2.27), it is in general a highly non-trivial task to investigate whether a fully consistent conformal field theory can be associated to that modular invariant. In particular one would like to compute the relevant $\mathrm{SL}_{2}(\mathbb{Z})$-representation. While the T-matrix for the extended theory is just the restriction of the original T-matrix to allowed orbits, for the S-matrix one only gets some constraints involving the S-matrix of the original 
theory, and determining those elements which involve two fixed points proves to be an intricate problem. However, recently a general class of solutions to this fixed point resolution problem was constructed [49, []; this construction yields in particular a general closed expression for the S-matrix.

\section{Operator Product Algebra from Fusion Rules}

The results of section 7 show that the operator product coefficients of primary fields are the normalizations of the corresponding three-point functions, but these normalizations cannot be determined from the Ward identities. With the help of four-point functions, on the other hand, one can compute the operator product coefficients. Indeed, for maximal $\mathcal{W}$ the coefficients $a_{I \bar{I}}$ that appear in the decomposition (1.42) of four-point correlators into three-point functions are of the form $a_{I \bar{I}}=a_{I} \delta_{\bar{I}, \pi I}$ for some permutation $\pi$. Moreover, provided that the chiral blocks are correctly normalized, the $a_{I}$ are just the products $C_{A B}{ }_{I} C_{I C D}$ of the relevant operator product coefficients. As a consequence, one can regard a conformal field theory as completely solved once all four-point functions of its primaries are known. These correlators, in turn, can be deduced from the fusion rules of the theory by using general analytic properties of the correlators to construct linear differential equations which they must satisfy. ${ }^{8}$ The independent solutions of these differential equations are the chiral blocks, which transform into each other under duality transformations (see (2.8)). Having obtained explicit formulæ for the blocks, one can therefore determine the duality matrices $\mathrm{F}$ and $\mathrm{B}$ and express the coefficients $a_{I}$, and hence the operator product coefficients, through the entries of these matrices [13, 39, 41].

To achieve this result in practice, one just exploits the following basic properties of (rational or quasi-rational, genus zero) conformal field theories:

- invariance of the vacuum vector $v_{\circ}$ under the projective $\mathfrak{s l}(2)$-algebra (1.20) (see section 4);

- existence of a closed associative operator algebra (1.37) whose structure 'constants' are of a specific form that is compatible with the $\mathcal{W}$-symmetry (section 6);

- factorization into chiral and antichiral blocks (section 7);

- independence of the chiral blocks.

First, one can invoke projective invariance to work with four-point correlators $\mathcal{G}(z, \bar{z})$ which are of the special form (1.41). Next, the closure of the operator product algebra implies that $\mathcal{G}$ can be written as a sum of products of three-point functions (compare the picture (2.5)), and because of the $\mathcal{W}$-symmetry the contributions from each 'intermediate' family $\left[\phi_{I}\right]$ combine to a block of definite analytic behavior. Furthermore, by the fact that the symmetry algebra is the direct $\operatorname{sum} \mathcal{W} \oplus \overline{\mathcal{W}}$ of two chiral halves, the $z$ - and $\bar{z}$-dependence factorize, which amounts to the decomposition (1.42). The number $M(=\bar{M}$ for maximally extended $\mathcal{W})$ of blocks in (1.42) is determined as

$$
M=\sum_{I} \mathrm{~N}_{A B}^{I} \mathrm{~N}_{I C D}
$$

with $I$ labelling the intermediate families $\left[\phi_{I}\right]$, through the fusion rule coefficients $\mathrm{N}_{A B}{ }^{C}$.

Now recall that the chiral blocks are, generically, not ordinary functions but multi-valued. When the chiral blocks $\mathcal{F}_{I}(z)$ are known, then requiring that $\mathcal{G}(z, \bar{z})$ becomes single-valued when $\bar{z}$ is taken to be the complex conjugate of $z$ - which can be encoded in the crossing symmetry

${ }^{28}$ Often these differential equations also follow from the presence of null vectors in the Verma modules of $\mathcal{W}$. An example is provided by the Knizhnik-Zamolodchikov equations which will be discussed in section 24 . 
relations (2.7) - determines the complex constants $a_{I \bar{I}}$ up to an over-all factor. By considering the collection of all four-point functions of primary fields (and fixing a definite normalization of each primary field), one can compute these over-all factors, too.

To determine the chiral blocks, one observes that according to the operator product expansion (1.37), $\phi_{i}(z, \bar{z}) \phi_{j}(w, \bar{w})$ depends on $z$ and $w$ as $(z-w)^{\alpha}$, with $\alpha$ determined by the conformal dimensions of the fields involved. More precisely,

$$
\mathcal{F}_{I}(z) \propto z^{-\Delta_{C}-\Delta_{D}+\tilde{\Delta}_{I}^{(C D)}} \quad \text { for } z \rightarrow 0
$$

Here $\Delta_{C}$ is the conformal dimension of the primary field $\phi_{C}$, while $\tilde{\Delta}_{I}^{(C D)}=\Delta_{I}^{(C D)}+\mu_{I}^{(C D)}$ is the conformal dimension of that field $\varphi_{I} \in\left[\phi_{I}\right]$, of grade $\mu_{I}^{(C D)}$, in the family of the primary $\phi_{I} \equiv \phi_{I}^{(C D)}$ which gives the leading contribution to the coupling between $\phi_{C}, \phi_{D}$ and $\left[\phi_{I}\right]$. The result (2.29) corresponds to the decomposition of $\mathcal{G}$ into three-point blocks as indicated in figure (2.5). Analogously, decomposing as in picture (2.6) yields different chiral blocks $\hat{\mathcal{F}}_{K}$ and $\check{\mathcal{F}}_{J}$ which behave as

$$
\begin{array}{ll}
\hat{\mathcal{F}}_{K} \propto(1-z)^{-\Delta_{C}-\Delta_{B}+\tilde{\Delta}_{K}^{(C B)}} & \text { for } z \rightarrow 1, \\
\check{\mathcal{F}}_{J} \propto\left(z^{-1}\right)^{\Delta_{C}-\Delta_{A}+\tilde{\Delta}_{J}^{(C A)}} & \text { for } z \rightarrow \infty
\end{array}
$$

with $\tilde{\Delta}_{J}^{(C B)}$ and $\tilde{\Delta}_{K}^{(C A)}$ defined analogously as $\tilde{\Delta}_{I}^{(C D)}$. (Thus the systems $\left\{\mathcal{F}_{I}\right\},\left\{\hat{\mathcal{F}}_{K}\right\}$ and $\left\{\check{\mathcal{F}}_{J}\right\}$ have diagonal monodromy around $z=0,1$ and $\infty$, respectively.) As already described in section 9 , the systems $\left\{\mathcal{F}_{I}\right\},\left\{\hat{\mathcal{F}}_{K}\right\}$ and $\left\{\breve{\mathcal{F}}_{J}\right\}$ of chiral blocks transform into each other upon analytic continuation. On the other hand, since they correspond to distinct couplings they must be algebraically independent; in technical terms, this amounts to the property that their Wronskian determinant must not vanish identically. According to elementary results from the theory of ordinary linear differential equations (see e.g. [111) it then follows that the chiral blocks $\mathcal{F}_{I}$ constitute the $M$ independent solutions of an $M$ th order differential equation. More precisely, the blocks obey

$$
\partial^{M} \mathcal{F}(z)+\sum_{m=0}^{M-1} h_{m}(z) \partial^{m} \mathcal{F}(z)=0
$$

with certain rational coefficient functions $h_{m}(z)$. As prescribed by the singular terms in the relevant operator products, the solutions of (2.31) have branch cut singularities at the points $z=0,1$ and $\infty$. It can happen that the coefficient functions $h_{m}(z)$ in (2.31) also possesses additional singular points, at which all of the solutions are regular; such singular points are known as apparent singularities.

The construction of the differential equation (2.31) is essentially equivalent to solving a Riemann monodromy problem, i.e. determine a collection of functions with prescribed monodromy. The Riemann monodromy problem always possesses a solution, provided that one allows for the possible presence of apparent singularities, around which the monodromy is trivial, in the differential equation. The general solution of (2.31) can be summarized in a so-called Riemann scheme $\mathrm{P}$, which specifies the positions of the singularities $z_{i}$ of the differential equation together with the exponents $\gamma_{I}^{i}$ at $z_{i}$, i.e. the roots of the $M$ th order algebraic equation for $\gamma^{(i)}$ that one obtains in lowest order in $z-z_{i}$ by making the power series ansatz $\mathcal{F}(z)=\left(z-z_{i}\right)^{\gamma^{(i)}} \sum_{p=0}^{\infty} a_{p}\left(z-z_{i}\right)^{p} \quad\left(a_{0} \neq 0\right.$; for $z_{i}=\infty, z-z_{i}$ is to be interpreted as $\left.z^{-1}\right)$. The Riemann scheme $\mathrm{P}$ does not, in general, determine uniquely the functions $\mathcal{F}_{I}(z)$. But one finds that as long as there are no apparent singularities, all of the parameters not specified by $\mathrm{P}$ 
(called accessory parameters) can be fixed by imposing the polynomial equations (2.9). When apparent singularities are present, the situation is more involved because only the positions and exponents of the real singularities of correlation functions are dictated by the operator products. In other words, the singularity structure of the four-point functions is fully known if and only if both the conformal weights of the primary fields (including the integer part) and - in the case of fusion rule coefficients larger than 1 - also the grades of the exchanged fields which are responsible for the leading singular behavior of a chiral block, are given. Once this is the case, the results for the blocks are unique provided that the differential equations satisfied by the four-point blocks do not possess any apparent singularities [13. On the other hand, the presence of apparent singularities spoils the uniqueness of the solution.

The position of an apparent singularity is almost arbitrary. There is only a mild restriction which results from the crossing symmetry [41]. The polynomial equations (2.9) do not impose any further restriction, as follows from the theory of isomonodromic deformations of differential equations. Namely, for arbitrarily chosen rational coefficient functions $f_{n}(z)$ the combinations $\mathcal{E}_{I}(z):=\sum_{n=0}^{M-1} f_{n}(z) \partial^{n} \mathcal{F}_{I}(z)$ and similarly defined functions $\hat{\mathcal{E}}_{K}, \check{\mathcal{E}}_{J}$ not only possess the same monodromy as the blocks $\mathcal{F}_{I}, \hat{\mathcal{F}}_{K}, \check{\mathcal{F}}_{J}$, but also the same fusing and braiding matrices (roughly, duality can be regarded as the 'square root' of monodromy). By varying the rational functions $f_{n}$, the position (and, generically, even the number) of apparent singularities can be varied freely. The positions of the apparent singularities of various distinct four-point functions are related, but only by the constraints that are implied by the crossing symmetry.

\section{Lecture 3: Kac-Moody Algebras}

This lecture is plain mathematics. In the final lecture 4 the mathematical structures studied here will be applied to conformal field theory.

\section{Cartan Matrices}

Kac-Moody algebras constitute a certain class of (generically infinite-dimensional) Lie algebras. Roughly, they are those Lie algebras which possess both a Cartan matrix (implying the existence of a triangular decomposition) and a Killing form. They include all finite-dimensional simple Lie algebras, and I will start by summarizing some pertinent features of those well-known algebras. A finite-dimensional simple Lie algebra $\mathfrak{g}=\mathfrak{g}(A)$ is characterized by its Cartan matrix A; the basic Lie bracket relations of $\mathfrak{g}$ can be expressed through $A$ as

$$
\begin{aligned}
& {\left[H^{\alpha}, H^{\beta}\right]=0} \\
& {\left[H^{\alpha}, E^{\beta}\right]=\left(\alpha^{\vee}, \beta\right) E^{\beta}} \\
& {\left[E^{\alpha}, E^{-\alpha}\right]=H^{\alpha}} \\
& {\left[E^{\alpha}, E^{\beta}\right]=e_{\alpha, \beta} E^{\alpha+\beta} \quad \text { for } \alpha \neq-\beta}
\end{aligned}
$$$$
\begin{aligned}
& {\left[H^{i}, H^{j}\right]=0} \\
& {\left[H^{i}, E_{ \pm}^{j}\right]= \pm A^{j i} E_{ \pm}^{j}} \\
& {\left[E_{+}^{i}, E_{-}^{j}\right]=\delta_{i j} H^{j}} \\
& \left(\operatorname{ad}_{E_{ \pm}^{i}}\right)^{1-A^{j i}} E_{ \pm}^{j}=0
\end{aligned}
$$

29 This definition is asymmetric in $i$ and $j$. In the literature the Cartan matrix is sometimes defined as the transpose of the matrix used here. 
in a so-called Cartan-Weyl basis and Chevalley-Serre basis, respectively. Let me explain some of the notation that is used here. When $\mathfrak{g}$ has rank $r, A$ is an $r \times r$-matrix, and $\mathfrak{g}$ has a basis $\left\{E^{\alpha}\right\} \cup\left\{H^{i} \mid i=1,2, \ldots, r\right\}$ obeying the relations (3.1A) (in addition to antisymmetry and the Jacobi identity, the two defining properties of a Lie bracket). The $r$ generators $H^{i}$ span an abelian Lie algebra, called the Cartan subalgebra $\mathfrak{g}_{\circ}$ of $\mathfrak{g}$; the $\operatorname{maps}_{H^{i}}: x \mapsto\left[H^{i}, x\right]$ are diagonalizable, and to each root, i.e. vector $\alpha=\left(\alpha^{i}\right)$ of eigenvalues of these maps, there corresponds a generator $E^{\alpha}$ of $\mathfrak{g}$, and one writes $H^{\alpha}:=\left(\alpha^{\vee}, H\right)$ with $\alpha^{\vee}:=2 \alpha /(\alpha, \alpha)$. Among the roots there is a special subset $\left\{\alpha^{(i)} \mid i=1,2, \ldots, r\right\}$, called the simple roots, through which all roots can be expressed as integral linear combinations with either only positive or only negative coefficients; the corresponding roots are called positive and negative roots, respectively. (Actually there are several distinct subsets of roots which can serve as systems of simple roots; but all of them are equivalent.) Writing $E^{ \pm \alpha^{(i)}}=: E_{ \pm}^{i}, \mathfrak{g}=\mathfrak{g}(A)$ is generated algebraically by $3 r$ generators $\left\{E_{ \pm}^{i}, H^{i} \mid i=1,2, \ldots, r\right\}$ (called the Chevalley generators of $\mathfrak{g}$ ) modulo the relations (3.1B). The relations in the last line of (3.1B) are called the Serre relations (the symbol $\left(\operatorname{ad}_{x}\right)^{n}$ is a shorthand for $\operatorname{ad}_{x} \circ \operatorname{ad}_{x} \circ \cdots \circ \operatorname{ad}_{x}$ ( $n$ factors), e.g. $\left.\left(\operatorname{ad}_{x}\right)^{3}(y) \equiv[x,[x,[x, y]]]\right)$, and the full set (3.1B) is known as the Chevalley-Serre relations. Cartan matrices are conveniently encoded into Dynkin diagrams: to each possible value of the label $i$ one associates a node or vertex, and for $i \neq j$ the nodes $i$ and $j$ are connected by $A^{i j} A^{j i}$ lines.

Of course, not any arbitrary square matrix qualifies as a Cartan matrix. In order that $\mathfrak{g}(A)$ is finite-dimensional and simple, the matrix $A$ must satisfy

$$
A^{i j} \in \mathbb{Z}, \quad A^{i i}=2, \quad A^{i j} \leq 0 \text { for } i \neq j, \quad A^{i j}=0 \Leftrightarrow A^{j i}=0,
$$

as well as

$$
\operatorname{det} A>0 \text {, }
$$

and must be indecomposable (i.e. must not be rearrangeable to block-matrix form by any simultaneous permutation of its rows and columns; decomposable matrices obeying (3.2) and (3.3) describe direct sums of simple Lie algebras). The analysis of these conditions leads to the following classification of all finite-dimensional simple Lie algebras:

$$
A_{r(r \geq 1),} \quad B_{r(r \geq 2)}, \quad C_{r(r \geq 3),} D_{r}(r \geq 4), \quad E_{6}, \quad E_{7}, \quad E_{8}, \quad F_{4}, G_{2} .
$$

Thus there are four infinite series, the classical Lie algebras $A_{r}, \ldots, D_{r}$, as well as the five exceptional simple Lie algebras $E_{6}, \ldots, G_{2}$. In particular there is a unique simple Lie algebra of rank one, namely $A_{1} \cong \mathfrak{s l}(2)$, with Cartan matrix $A\left(A_{1}\right)=2$, and there are three simple Lie algebras of rank two, namely $A_{2} \cong \mathfrak{s l}(3), B_{2} \equiv C_{2} \cong \mathfrak{s o}(5)$ and $G_{2}$, with Cartan matrices

$$
A\left(A_{2}\right)=\left(\begin{array}{rr}
2 & -1 \\
-1 & 2
\end{array}\right), \quad A\left(B_{2}\right)=\left(\begin{array}{rr}
2 & -2 \\
-1 & 2
\end{array}\right), \quad A\left(G_{2}\right)=\left(\begin{array}{rr}
2 & -3 \\
-1 & 2
\end{array}\right) .
$$

By construction, the space spanned by the $\mathfrak{g}$-roots $\alpha$ is the space $\mathfrak{g}_{\circ}^{\star}$ dual to the Cartan subalgebra $\mathfrak{g}_{\circ} \cdot \mathfrak{g}_{\circ}^{\star}$, called the weight space of $\mathfrak{g}$, is an $r$-dimensional vector space with euclidean scalar product $(\cdot, \cdot)$; the simple roots, and likewise the simple coroots $\alpha^{(i) \vee}:=2 \alpha^{(i)} /\left(\alpha^{(i)}, \alpha^{(i)}\right)$,

30 By '(algebraically) generated' - as opposed to '(linearly) spanned' - one means that the elements of the Lie algebra $\mathfrak{g}$ are obtained as arbitrary linear combinations of arbitrary (multiple) Lie brackets of the basic generators. Such a characterization of the Lie algebra $\mathfrak{g}$, not to be confused with the description of $\mathfrak{g}$ as the linear span of a basis, is called a presentation of $\mathfrak{g}$ by generators modulo relations. 
form a basis of $\mathfrak{g}_{\circ}^{\star}$. The entries of the Cartan matrix encode the non-orthonormality of these bases; more precisely,

$$
A^{i j}=\left(\alpha^{(i) \vee}, \alpha^{(j)}\right) \equiv 2\left(\alpha^{(i)}, \alpha^{(j)}\right) /\left(\alpha^{(j)}, \alpha^{(j)}\right) .
$$

Further properties of the root system, i.e. the collection of all roots of $\mathfrak{g}$, are the following. The only multiple of a root $\alpha$ that is again a root is $-\alpha$. Also, for any pair $\alpha, \beta$ of roots, the linear combination $\alpha+m \beta$ is a root for a set of consecutive integers $m$, but not for any other value of $m$; for pairs of simple roots, this root string can be directly read off the Serre relations. For the lengths of roots at most two different values are possible; the relative length squared of a longer and a shorter root is given by $\max \left\{\left|A^{i j}\right| \mid i \neq j\right\}$. In particular, when $A^{i j} \in\{0,-1\}$ for $i \neq j$ (in which case $\mathfrak{g}$ is said to be simply laced), then all roots have the same length. Also, when different root lengths do occur, then this already happens among the simple roots, and each of the two sets of simple roots of equal length corresponds to a connected sub-diagram of the Dynkin diagram. (Also, in order that the Dynkin diagram specifies the Lie algebra $\mathfrak{g}$ uniquely, one supplements the multiple link which connects these two sub-diagrams by an arrow pointing from the longer to the shorter simple roots.)

The Killing form of $\mathfrak{g}$ associates to any two elements $x$ and $y$ of $\mathfrak{g}$ the number

$$
\kappa(x, y) \equiv(x \mid y):=\operatorname{tr}\left(\operatorname{ad}_{x} \circ \operatorname{ad}_{y}\right) .
$$

It is bilinear, symmetric, and satisfies $\kappa(x,[y, z])=\kappa([x, y], z)$. Conversely, any map with these properties equals the Killing form up to a multiplicative constant. When restricted to the Cartan subalgebra $\mathfrak{g}_{\circ}$, the Killing form induces by duality an inner product on the weight space $\mathfrak{g}_{\circ}^{\star}$, which, when suitably normalized, coincides with the euclidean product on $\mathfrak{g}_{\circ}^{\star}$ that was already used above.

Another important basis of the weight space $\mathfrak{g}_{\circ}^{\star}$ consists of the fundamental weights $\Lambda_{(i)}$, which are defined by the requirement that

$$
\left(\Lambda_{(i)}, \alpha^{(j)^{\vee}}\right)=\delta_{i}^{j}
$$

for $i, j=1,2, \ldots, r$. This basis plays a special rôle in the representation theory of $\mathfrak{g}$, or more precisely, for its highest weight modules (the concept of highest weight modules was already discussed in the context of chiral algebras in section 3, and will be dealt with in more detail in section 19 below). Namely, among the irreducible highest weight modules $\mathcal{H}_{\Lambda}$ of $\mathfrak{g}$, precisely those are finite-dimensional whose highest weight $\Lambda$ is dominant integral, i.e. is a non-negative integral linear combination of the fundamental weights $\Lambda_{(i)}$. Moreover, each such module can be obtained by forming tensor products of just a few special highest weight modules, for all of which the highest weight is a fundamental weight.

The Weyl group $W$ of the Lie algebra $\mathfrak{g}$ is a finite group which is generated by the reflections $r_{i}, i \in\{1,2, \ldots, r\}$, in the weight space $\mathfrak{g}_{\circ}^{\star}$ of $\mathfrak{g}$ about the hyperplanes through the origin which are perpendicular to the simple roots $\alpha^{(i)}$. On the fundamental weights $\Lambda_{(j)}$ these reflections act as

$$
r_{i}: \quad \Lambda_{(j)} \mapsto \Lambda_{(j)}-\delta_{i, j} \sum_{k} A^{i k} \Lambda_{(k)}
$$

Each element $w \in W$ can be written (non-uniquely) as a product of the reflections $r_{i}$. The minimal number of $r_{i}$ that is needed to form such a Weyl word for $w$ is called the length $\ell(w)$ of $w$, and $(-1)^{\ell(w)}=: \operatorname{sign}(w)$ is called the sign of $w$. 


\section{Symmetrizable Kac-Moody Algebras}

A considerable portion of the description above directly generalizes to a much larger class of Lie algebras. Recall that the finite-dimensional simple Lie algebras are obtained when imposing indecomposability as well as the properties (3.2) and (3.3) of $A$; in particular the $\operatorname{rank}$ of $A$ is equal to $r$. One arrives at more general Lie algebras $\mathfrak{g}$ when one stipulates again that $\mathfrak{g}=\mathfrak{g}(A)$ is algebraically generated by Chevalley generators $E_{ \pm}^{i}, H^{i}$ modulo the relations (3.1B), with the matrix $A$ still satisfying (3.2), but relaxes the condition (3.3) on the determinant of $A$. The algebras obtained this way are known as Kac-Moody Lie algebras, and $A$ as a generalized Cartan matrix. All Kac-Moody algebras, except for the simple ones described above, are infinite-dimensional (for the case of affine Kac-Moody algebras, this follows from their realization that I will present in the next section).

Remarkably, even removing the condition (3.3) altogether does not destroy much of the power and beauty of these Lie algebras and their representation theory. But to keep some specific nice properties, it is necessary to restrict oneself to a (still comprehensive) subclass, the so-called symmetrizable Kac-Moody algebras. These are characterized by the existence of a non-degenerate diagonal matrix $\mathrm{D}$ such that the matrix $\mathrm{D} A$ is symmetric. A symmetrizable Kac-Moody algebra is simple (that is, it does not possess any non-trivial ideal, i.e. subalgebra $\mathfrak{h}$ such that $[\mathfrak{h}, \mathfrak{g}] \subseteq \mathfrak{h}$, and has dimension larger than 1 ), if an only if $\operatorname{det} A \neq 0$.

Every symmetrizable Kac-Moody algebra possesses a bilinear symmetric invariant form $(\cdot \mid \cdot)$ - in the finite-dimensional case this is the Killing form (3.7). However, when $\mathfrak{g}$ is strictly defined as above, this bilinear form is degenerate as soon as the symmetrized Cartan matrix D $A$ has a zero eigenvalue. To make the form non-degenerate, one needs to enlarge the Cartan subalgebra $\mathfrak{g}_{\circ}$ of $\mathfrak{g}$ by so-called outer derivations $D$. More precisely, to every zero eigenvalue of $A$ there is associated an independent central element $K^{a} \in \mathfrak{g}$ which is a suitable linear combination of the generators $H^{i}$. For each $K^{a}$ one must introduce an independent derivation $D^{a}$; this can be done in such a way that $\left(K^{a} \mid D^{b}\right)=\delta^{a b}$ and $\left(K^{a} \mid K^{b}\right)=0=\left(D^{a} \mid D^{b}\right)$, and then $(\cdot \mid \cdot)$ is non-degenerate. It is usually this enlarged Lie algebra that is referred to as the Kac-Moody algebra $\mathfrak{g}=\mathfrak{g}(A)$ associated to $A$.

When (3.3) is not removed completely, but only relaxed to the requirement

$$
\operatorname{det} A_{\{i\}}>0 \text { for all } i=0,1, \ldots, r
$$

for the matrices $A_{\{i\}}$ that are obtained from $A$ by deleting the $i$ th row and $i$ th column (and when $A$ is indecomposable), then $A$ is called an affine Cartan matrix, and $\mathfrak{g}=\mathfrak{g}(A)$ an affine Lie algebra. In (3.10) I changed the labelling convention for the entries of $A$ : they now take values in $\{0,1, \ldots, r\}$, so that $A$ is an $(r+1) \times(r+1)$-matrix; note that the validity of $(3.10)$ implies that the rank of $A$ is at least $r$. One can also characterize an affine Cartan matrix by the requirement that the symmetrized Cartan matrix $\mathrm{D} A$ is positive semidefinite, but not positive definite. (If $\mathrm{D} A$ is positive definite, then $\mathfrak{g}(A)$ is a finite-dimensional simple Lie algebra.) The center of an affine Lie algebra is one-dimensional, and correspondingly one includes into the definition of $\mathfrak{g}$ an outer derivation $D$.

Once the classification of simple Lie algebras up to some rank is known, classifying the affine Lie algebras with that rank is straightforward. For instance, just like for $r=1$ requiring $\operatorname{det} A>$ 0 immediately leads to the three simple rank-two Cartan matrices (3.5), the requirement that $A$ has one positive and one zero eigenvalue yields two rank-one affine algebras, denoted by $A_{1}^{(1)}$ 
and $A_{1}^{(2)}: \square$

$$
A\left(A_{1}^{(1)}\right)=\left(\begin{array}{rr}
2 & -2 \\
-2 & 2
\end{array}\right), \quad A\left(A_{1}^{(2)}\right)=\left(\begin{array}{rr}
2 & -4 \\
-1 & 2
\end{array}\right) .
$$

For $r>1$, the crucial observation is that by deleting the $i$ th row and $i$ th column $(i \in\{0,1, \ldots, r\}$ arbitrary) from an affine Cartan matrix, one must produce the Cartan matrix of a direct sum of finite-dimensional simple Lie algebras. This leads to the following classification. There are seven infinite series of affine Lie algebras, and in addition nine isolated cases; they are denoted by

$$
\begin{aligned}
& A_{r}^{(1)}(r \geq 2), \quad B_{r}^{(1)}(r \geq 3), \quad C_{r}^{(1)}(r \geq 2), D_{r}^{(1)}(r \geq 4), B_{r}^{(2)}(r \geq 3), C_{r}^{(2)}(r \geq 2), \tilde{B}_{r}^{(2)}(r \geq 2), \\
& A_{1}^{(1)}, \quad E_{6}^{(1)}, E_{7}^{(1)}, E_{8}^{(1)}, F_{4}^{(1)}, G_{2}^{(1)}, A_{1}^{(2)}, F_{4}^{(2)}, G_{2}^{(3)} .
\end{aligned}
$$

The affine algebras are thus all denoted by symbols $X_{r}^{(\ell)}$, with $X_{r}$ the symbol for one of the simple Lie algebras (3.4) and $\ell \in\{1,2,3\}$. The algebras with $\ell=1$ are called untwisted affine algebras, while those with $\ell=2,3$ are the twisted ones. For the time being, the distinction between these two types of algebras is only a matter of nomenclature, but the reason for this choice of terminology will become clear later on.

\section{Affine Lie Algebras as Centrally Extended Loop Algebras}

The description of a Kac-Moody algebra $\mathfrak{g}$ in terms of generators $\left\{E_{ \pm}^{i}, H^{i}\right\}$ and relations (3.1B) encodes the structure of $\mathfrak{g}$ in a very compact form, ${ }^{3}$ but it is in fact not too transparent. (For instance it is difficult to deduce the root system, and it even requires some effort to decide whether $\mathfrak{g}$ is finite-dimensional.) But affine Kac-Moody algebras possess a specific realization which for many purposes is much more convenient and which arises naturally in conformal field theory.

Consider the space $\overline{\mathfrak{g}}_{\text {loop }}$ of analytic mappings from the circle $S^{1}$ to some Lie algebra $\overline{\mathfrak{g}}$. (For future convenience I denote the Lie algebra to start with by $\overline{\mathfrak{g}}$ rather than $\mathfrak{g}$.) Fourier analysis shows that when $S^{1}$ is regarded as the unit circle in the complex plane with coordinate $z=\mathrm{e}^{2 \pi \mathrm{it}}$, then a (topological basis of the function space $\overline{\mathfrak{g}}_{\text {loop }}$ is given by $\left\{T_{n}^{a} \mid a=1,2, \ldots, \operatorname{dim} \overline{\mathfrak{g}} ; n \in \mathbb{Z}\right\}$, where

$$
T_{n}^{a}:=\bar{T}^{a} \otimes z^{n} \equiv \bar{T}^{a} \otimes \mathrm{e}^{2 \pi \mathrm{in} t}
$$

with $z \in S^{1}$ and $\left\{\bar{T}^{a} \mid a=1,2, \ldots, \operatorname{dim} \overline{\mathfrak{g}}\right\}$ a basis of $\overline{\mathfrak{g}}$. Moreover, $\overline{\mathfrak{g}}_{\text {loop }}$ inherits a natural bracket operation from $\overline{\mathfrak{g}}$ with respect to which it becomes a Lie algebra itself, namely

$$
\left[T_{m}^{a}, T_{n}^{b}\right]:=\left[\bar{T}^{a}, \bar{T}^{b}\right] \otimes\left(z^{m} z^{n}\right)=\sum_{c=1}^{\operatorname{dim} \overline{\mathfrak{g}}} f_{c}^{a b} \bar{T}^{c} \otimes z^{m+n}=\sum_{c=1}^{\operatorname{dim} \overline{\mathfrak{g}}} f_{c}^{a b} T_{m+n}^{c} .
$$

\footnotetext{
${ }^{31}$ The value $A^{12} A^{21}=4$ is an exception: for every other affine Lie algebra one has $A^{i j} A^{j i} \leq 3$ for all $i, j$.

${ }^{32}$ For $\ell=2,3$ several different conventions are in use. The notation adopted here is taken from [42]; a rather different one is used in 777 .

33 The most elegant formulation 77 is to start with a Cartan subalgebra of sufficiently large dimension and describe $\mathfrak{g}$ as the Lie algebra generated modulo (3.1 B), divided by its maximal ideal that intersecting non-trivially the Cartan subalgebra.

${ }^{34}$ A topological or analytic basis, sometimes also called Hilbert space basis, of a vector space $V$ is characterized by the property that the closure in the topology of $V$ of the set of all finite linear combinations of basis vectors is the whole space $V$. In contrast, an ordinary vector space basis of $V$ has the property that every vector in $V$ is a finite linear combination of basis vectors.
} 
Accordingly, $\overline{\mathfrak{g}}_{\text {loop }}$ is called the loop algebra over $\overline{\mathfrak{g}}$. The subset of the loop algebra that is spanned by the generators $T_{0}^{a}$ is a Lie subalgebra, called the zero mode subalgebra of $\overline{\mathfrak{g}}_{\text {loop }}$; it is isomorphic to $\overline{\mathfrak{g}}$.

Now let $\overline{\mathfrak{g}}=X_{r}$ be a finite-dimensional simple Lie algebra. Its loop algebra $\overline{\mathfrak{g}}_{\text {loop }}$, which is infinite-dimensional, turns out to be closely related to the affine algebra $\mathfrak{g}=X_{r}^{(1)}$. 阿 However, unlike affine Lie algebras, $\overline{\mathfrak{g}}_{\text {loop }}$ has a trivial center (and also does not possess any interesting representations). But starting from $\overline{\mathfrak{g}}_{\text {loop }}$ one can obtain a Lie algebra which does possess a center, by a canonical construction known as central extension. Namely, by simply adjoining $\ell$ additional generators $K^{j}, j=1,2, \ldots, \ell$, to a basis $\left\{T^{a}\right\}$ of an arbitrary Lie algebra $\mathfrak{g}$, one constructs an algebra $\hat{\mathfrak{g}}$ whose dimension exceeds the dimension of $\mathfrak{g}$ by $\ell$ and for which the $K^{j}$ are central, as follows. One keeps the original values $f_{c}^{a b}$ of those structure constants which involve only the generators $\hat{T}^{a}$ and imposes the relations $\left[K^{i}, K^{j}\right]=0$ and $\left[\hat{T}^{a}, K^{j}\right]=0$ for all $i, j$ and all $a$. Here by $\hat{T}^{a}$ I denote the image of the generator $T^{a}$ of $\mathfrak{g}$ in the extended algebra $\hat{\mathfrak{g}}$. The general form of the brackets among these generators $\hat{T}^{a}$ reads $\left[\hat{T}^{a}, \hat{T}^{b}\right]=$ $\sum_{c} f_{c}^{a b} \hat{T}^{c}+\sum_{i=1}^{\ell} f_{i}^{a b} K^{i}$. The new additional structure constants $f_{i}^{a b}$ are not arbitrary, but are restricted by the Jacobi identity. Clearly, one should count only those extensions as genuine central extensions for which $\hat{\mathfrak{g}}$ is not just the direct sum of $\mathfrak{g}$ and an abelian Lie algebra; such a direct sum is certainly obtained when $f_{i}^{a b} \equiv 0$, but this is typically not the only choice for which this happens.

It is in general a difficult cohomological question whether a given Lie algebra allows for non-trivial central extensions or not. But it is quite straightforward to see that finite-dimensional simple Lie algebras $\overline{\mathfrak{g}}=X_{r}$ do not possess non-trivial central extensions, whereas their loop algebras $\overline{\mathfrak{g}}_{\text {loop }}$ possess indeed a unique non-trivial extension by a single central generator $K$, with brackets

$$
\left[K, \hat{T}_{n}^{a}\right]=0, \quad\left[\hat{T}_{m}^{a}, \hat{T}_{n}^{b}\right]=\sum_{c=1}^{\operatorname{dim} \overline{\mathfrak{g}}} f_{c}^{a b} \hat{T}_{m+n}^{c}+m \delta_{m+n, 0} \bar{\kappa}^{a b} K
$$

here $\bar{\kappa}^{a b}=\bar{\kappa}\left(\bar{T}^{a}, \bar{T}^{b}\right)$, with $\bar{\kappa}$ the Killing form (3.7) of $\overline{\mathfrak{g}}$. Moreover, the untwisted affine Lie algebra $\mathfrak{g}=X_{r}^{(1)}$ is obtained from this centrally extended loop algebra $\widehat{\mathfrak{g}_{\text {loop }}}$ by including a derivation $D$, i.e. a generator with Lie brackets

$$
\left[D, \hat{T}_{m}^{a}\right]=m \hat{T}_{m}^{a}, \quad[D, K]=0 .
$$

(Thus $D$ measures the mode number $m$ and hence provides a $\mathbb{Z}$-grading, which is of precisely the same form as that of the Virasoro operator $-L_{0}$ in the case of chiral algebras $\mathcal{W}$, cf. equation (1.7).) In short, the vector space structure of $\mathfrak{g}$ is

$$
\mathfrak{g}=\hat{\mathfrak{g}} \oplus \mathbb{C} D=\mathbb{C}\left[z, z^{-1}\right] \otimes_{\mathbb{C}} \overline{\mathfrak{g}} \oplus \mathbb{C} K \oplus \mathbb{C} D
$$

Just like for loop algebras, the zero modes $\hat{T}_{0}^{a}$ of $\mathfrak{g}$ span a subalgebra which is isomorphic to the simple Lie algebra $\overline{\mathfrak{g}}$; this is called the horizontal subalgebra of $\mathfrak{g}$.

\footnotetext{
35 The notation in the lists (3.4) and (3.12) is chosen in such a way that $\mathfrak{g}=X_{r}^{(1)}$ with $X \in\{A, B, C, D, E\}$ precisely corresponds to the simple Lie algebra $\overline{\mathfrak{g}}=X_{r}$.
} 
Upon choosing a Cartan-Weyl basis $\left\{\bar{T}^{a}\right\}=\left\{H^{i} \mid i=1,2, \ldots, r\right\} \cup\left\{E^{\bar{\alpha}}\right\}$ of $\overline{\mathfrak{g}} \equiv \overline{\mathfrak{g}}(\bar{A})$, the defining Lie bracket relations (3.1A) of $\mathfrak{g}$ take the form

$$
\begin{aligned}
& {\left[H_{m}^{i}, H_{n}^{j}\right]=m(\overline{\mathrm{D}} \bar{A})^{i j} \delta_{m+n, 0} K, \quad\left[H_{m}^{i}, E_{n}^{\bar{\alpha}}\right]=\bar{\alpha}^{i} E_{m+n}^{\bar{\alpha}},} \\
& {\left[E_{m}^{\bar{\alpha}}, E_{n}^{\bar{\beta}}\right]=e_{\bar{\alpha}, \bar{\beta}} E_{m+n}^{\bar{\alpha}+\bar{\beta}} \quad \text { for } \bar{\alpha}+\bar{\beta} \text { a } \overline{\mathfrak{g}}-\text { root },} \\
& {\left[E_{n}^{\bar{\alpha}}, E_{-n}^{-\bar{\alpha}}\right]=\sum_{i=1}^{r} \bar{\alpha}_{i} H_{0}^{i}+n K .}
\end{aligned}
$$

Here $m, n \in \mathbb{Z}, i, j \in\{1,2, \ldots, r\}$, and $\bar{\alpha}, \bar{\beta}$ are arbitrary $\overline{\mathfrak{g}}$-roots.

So far only the untwisted affine algebras have been obtained. But along similar lines one can realize the twisted ones as well. The only modification is to give up the single-valuedness of the maps $f$ from $S^{1}$ to $\overline{\mathfrak{g}}$ that were used in the loop construction above. More precisely, the twisted affine Lie algebras are obtained when instead of $f\left(\mathrm{e}^{2 \pi \mathrm{i}} z\right)=f(z)$ one imposes the twisted boundary conditions

$$
x \otimes f\left(\mathrm{e}^{2 \pi \mathrm{i}} z\right)=\omega(x) \otimes f(z)
$$

for all $x \in \overline{\mathfrak{g}}$, where $\omega$ is an automorphism of the horizontal subalgebra $\overline{\mathfrak{g}}$ of finite order $N$. In other words, $f$ is now no longer a function on the circle $S^{1}$, but rather on an $N$-fold covering of $S^{1}$. However, when performing this construction for two automorphisms $\omega$ and $\omega^{\prime}$ for which $\omega^{-1} \omega^{\prime}$ is an inner automorphism of $\overline{\mathfrak{g}}$, one obtains two realizations of one and the same abstract Lie algebra; in particular, when $\omega$ is inner, then one gets a realization of an untwisted algebra which differs from the realization (3.18). As a consequence, the twisted affine Lie algebras correspond in fact to equivalence classes of outer modulo inner automorphisms of $\overline{\mathfrak{g}}$; in each such class there is a distinguished representative which is induced by a symmetry of the Dynkin diagram of $\overline{\mathfrak{g}}$.

\section{The Triangular Decomposition of Affine Lie Algebras}

For any affine Lie algebra $\mathfrak{g}$ one can determine a triangular decomposition of $\mathfrak{g}$ that is analogous to (1.12) for chiral algebras. One first observes that a Cartan subalgebra $\mathfrak{g}_{\circ}$ of $\mathfrak{g}$ is spanned by $\left\{K, D, H_{0}^{i} \mid i=1,2, \ldots, r\right\}$. The roots (i.e., vectors of eigenvalues) with respect to $\left(H_{0}, K, D\right)$ are given by

$$
\alpha=(\bar{\alpha}, 0, n) \quad \text { for } \quad \bar{\alpha} \in \bar{\Phi}, n \in \mathbb{Z} \quad \text { and } \quad \alpha=(0,0, n) \text { for } n \in \mathbb{Z} \backslash\{0\},
$$

where $\bar{\Phi}=\{\bar{\alpha}\}$ denotes the root system of $\overline{\mathfrak{g}}$. These roots correspond to the generators $E_{n}^{\bar{\alpha}}$ with $n \in \mathbb{Z}$ and to $H_{n}^{j}$ with $n \neq 0$, respectively. While the roots $(\bar{\alpha}, 0, n)$ are non-degenerate, i.e. appear with multiplicity one, the roots $(0,0, n)$ do not depend on the label $j$ of $H_{n}^{j}$ and hence have multiplicity $r$. The degenerate roots are all integral multiples of $\delta:=(0,0,1)$. Also note that the roots of the derived algebra $[\mathfrak{g}, \mathfrak{g}]=: \hat{\mathfrak{g}}$ are all infinitely degenerate, because in $\hat{\mathfrak{g}}$ there is no Cartan subalgebra generator that is able to distinguish between different labels $n$. Thus another rôle of the derivation $D$ is to avoid such infinite multiplicities.

Having made a choice for distinguishing between positive and negative roots of $\overline{\mathfrak{g}}$, a system of positive roots $(\alpha>0)$ of $\mathfrak{g}$ can be chosen to consist of those roots $(\bar{\alpha}, 0, n)$ for which either $n>0$ and $\bar{\alpha} \in \bar{\Phi}$ is an arbitrary $\overline{\mathfrak{g}}$-root or zero, or else $n=0$ and $\bar{\alpha}$ is a positive $\overline{\mathfrak{g}}$-root. The remaining roots are then the negative ones $(\alpha<0)$. Together with $\mathfrak{g}_{\circ}$, the subalgebras $\mathfrak{g}_{+}:=\operatorname{span}\left\{E^{\alpha} \mid \alpha>0\right\}$ and $\mathfrak{g}_{-}:=\operatorname{span}\left\{E^{-\alpha} \mid \alpha>0\right\}$ then provide a triangular decomposition

$$
\mathfrak{g}=\mathfrak{g}_{+} \oplus \mathfrak{g}_{\circ} \oplus \mathfrak{g}_{-}
$$


of $\mathfrak{g}$, i.e. satisfy $\left[\mathfrak{g}_{ \pm}, \mathfrak{g}_{\circ} \oplus \mathfrak{g}_{ \pm}\right] \subseteq \mathfrak{g}_{ \pm}$and $\left[\mathfrak{g}_{+}, \mathfrak{g}_{-}\right] \subseteq \mathfrak{g}_{\circ}$, analogous to (1.13)

The simple roots $\alpha^{(i)}$ of $\mathfrak{g}$ provide a basis of the root space such that in the expansion $\alpha=\sum_{i=0}^{r} b_{i} \alpha^{(i)}$ one has, for all $i=0,1, \ldots, r, b_{i} \in \mathbb{Z}_{\geq 0}$ if $\alpha>0$ and $b_{i} \in \mathbb{Z}_{\leq 0}$ if $\alpha<0$. With the above choice of positive roots, the simple roots are

$$
\alpha^{(i)}=\left(\bar{\alpha}^{(i)}, 0,0\right) \equiv \bar{\alpha}^{(i)} \quad \text { for } i=1,2, \ldots, r \quad \text { and } \quad \alpha^{(0)}=(-\bar{\theta}, 0,1)=\delta-\bar{\theta},
$$

where $\bar{\alpha}^{(i)}$ are the simple roots of the horizontal subalgebra $\overline{\mathfrak{g}}$ and $\bar{\theta}$ is the highest root of $\overline{\mathfrak{g}}$. For each $i \in\{0,1, \ldots, r\}$ the generator $E^{\alpha^{(i)}}$ corresponding to a simple root is precisely the generator that in the Chevalley-Serre formulation is denoted by $E_{+}^{i}$. The relation to the $E_{n}^{\bar{\alpha}}$-notation is thus

$$
E_{+}^{i}=E_{0}^{\bar{\alpha}^{(i)}} \quad \text { for } i=1,2, \ldots, r, \quad E_{+}^{0}=E_{1}^{-\bar{\theta}} .
$$

\section{Representation Theory}

Every Kac-Moody algebra $\mathfrak{g}$ has a triangular decomposition $\mathfrak{g}=\mathfrak{g}_{+} \oplus \mathfrak{g}_{\circ} \oplus \mathfrak{g}_{-}$similar to the one described above. Correspondingly a particularly interesting class of modules (representation spaces) $V$ of $\mathfrak{g}$ are those which have a basis on which the whole Cartan subalgebra $\mathfrak{g}_{\circ}$ acts diagonally, and hence possess a decomposition $V=\bigoplus_{\lambda} V_{(\lambda)}$ into subspaces such that

$$
R\left(H^{i}\right) v_{\lambda}=\lambda^{i} \cdot v_{\lambda}
$$

for all $v_{\lambda} \in V_{(\lambda)}$. The vectors $\lambda \equiv\left(\lambda^{i}\right)_{i}$ are called the weights of the module $V$; they are elements of the weight space $\mathfrak{g}_{0}^{\star}$, and the numbers $\lambda^{i}$ are their components in the basis furnished by the fundamental weights $\Lambda_{(i)}$.

A maximal weight $\Lambda$ satisfies by definition $R\left(E^{\alpha}\right) v_{\Lambda}=0$ for all positive roots $\alpha$ and all $v_{\Lambda} \in V_{(\Lambda)}$. A module which has exactly one weight $\Lambda$ with this property is called a highest weight module and is denoted by $V_{\Lambda}$. All other elements of $V_{\Lambda}$ can be obtained by applying step operators for negative roots to $v_{\Lambda}$, i.e. every $v \in V_{\Lambda}$ is contained in $\mathfrak{U}\left(\mathfrak{g}_{-}\right) v_{\Lambda}$, where $\mathfrak{U}$ denotes the universal enveloping algebra.

A Kac-Moody algebra $\mathfrak{g}$ is algebraically generated by its subalgebras $\mathfrak{g}_{(i)}$ that are associated to the simple $\mathfrak{g}$-roots, i.e. $\mathfrak{g}_{(i)}=\operatorname{span}\left\{E_{ \pm}^{i}, H^{i}\right\}$ (together with the derivations, but these do not concern us here). From $(3.1 \mathrm{~B})$ one reads off that $\mathfrak{g}_{(i)} \cong \mathfrak{s l}(2)$ for each $i$; one can therefore reduce many issues in the representation theory of $\mathfrak{g}$ to that of the simple Lie algebra $\mathfrak{s l}(2)$. A particularly important application is the following. All non-trivial $\mathfrak{g}$-modules are infinitedimensional, but in favorable circumstances the subspaces into which an irreducible highest weight module $\mathcal{H}_{\Lambda}$ decomposes with respect to the subalgebras $\mathfrak{g}_{(i)}$ are all finite-dimensional. (Loosely speaking, such $\mathfrak{g}$-modules are 'less infinite-dimensional' than others.) This happens precisely when the highest weight $\Lambda=\sum_{i} \Lambda^{i} \Lambda_{(i)}$ of $\mathcal{H}_{\Lambda}$ is dominant integral, that is, a positive integral linear combination of fundamental weights, i.e.

$$
\Lambda^{i} \equiv\left(\Lambda, \alpha^{(i)^{\vee}}\right) \in \mathbb{Z}_{\geq 0} \quad \text { for all } i
$$

(i.e. for $i=0,1, \ldots, r$ in the case of affine Lie algebras).

For affine $\mathfrak{g}$ it is convenient to write $\mathfrak{g}$-weights (cf. equation $([3.20))$ as triples $\lambda=(\bar{\lambda}, k, m)$ with $k$ the eigenvalue of the central element $K$ and $m$ the grade, i.e. the eigenvalue of the 
derivation $D$. Then for untwisted $\mathfrak{g}$, the fundamental weights are

$$
\Lambda_{(i)}=\left(\bar{\Lambda}_{(i)}, \frac{1}{2}(\bar{\theta}, \bar{\theta}) a_{i}^{\vee}, 0\right) \text { for } i=1,2, \ldots, r \quad \text { and } \quad \Lambda_{(0)}=\left(0, \frac{1}{2}(\bar{\theta}, \bar{\theta}), 0\right) .
$$

Here $a_{i}^{\vee}$ are the dual Coxeter labels, defined as the coefficients of $\bar{\theta}^{\vee} \equiv 2 \bar{\theta} /(\bar{\theta}, \bar{\theta})$ (the highest coroot) in the basis of simple coroots of $\overline{\mathfrak{g}}$. Also, the property (3.25) means, first, that the horizontal part $\bar{\Lambda}$ of $\Lambda$ is a dominant integral $\overline{\mathfrak{g}}$-weight, and second, that

$$
k^{\vee}:=2 k /(\bar{\theta}, \bar{\theta}),
$$

called the level of $\mathcal{H}_{\Lambda}$, is a non-negative integer; and third, there is the bound

$$
0 \leq \sum_{i=1}^{r} a_{i}^{\vee} \bar{\Lambda}^{i} \equiv\left(\bar{\Lambda}, \bar{\theta}^{\vee}\right) \leq k^{\vee}
$$

The precise structure of an irreducible highest weight module $\mathcal{H}_{\Lambda}$ strongly depends on whether the highest weight $\Lambda$ is dominant integral or not. For generic $\Lambda$ already the Verma module $\mathcal{V}_{\Lambda}$, which is isomorphic to $\mathfrak{U}\left(\mathfrak{g}_{-}\right) v_{\Lambda}$, without any additional relations (compare equation (1.18)), is irreducible. In contrast, for dominant integral $\Lambda$ the Verma module contains null vectors. The entire information on the null vector structure of $\mathcal{V}_{\Lambda}$ is encoded in the weight $\Lambda$ : all null vectors can be obtained by acting with $\mathfrak{U}\left(\mathfrak{g}_{-}\right)$on the so-called primitive null vectors

$$
v^{(i)}:=\left(E^{-\alpha^{(i)}}\right)^{\Lambda^{i}+1} v_{\Lambda}
$$

where $i$ can take all possible values, i.e. $i \in\{0,1, \ldots, r\}$ for affine algebras. The irreducible module $\mathcal{H}_{\Lambda}$ is obtained from the Verma module $\mathcal{V}_{\Lambda}$ by taking the quotient with respect to the submodule spanned by all null vectors. For affine $\mathfrak{g}$, the quotienting by the null vectors $v^{(i)}$ with $i=1,2, \ldots, r$ can be implemented by just restricting to irreducible modules rather than Verma modules with respect to the horizontal subalgebra $\overline{\mathfrak{g}}$ of $\mathfrak{g}$; also, one has $v^{(0)}=\left(E^{-\alpha^{(0)}}\right)^{\Lambda^{0}+1} v_{\Lambda}=$ $\left(E_{-1}^{\bar{\theta}}\right)^{k^{\vee}-\left(\bar{\theta}^{\vee}, \bar{\Lambda}\right)+1} v_{\Lambda}$.

\section{Characters}

Precisely as in the case of the chiral algebras $\mathcal{W}$ that arise in conformal field theory (see section 10), one can encode the information contained in the weight system of a module $V$ of a Kac-Moody algebra $\mathfrak{g}$ in its character

$$
\chi_{V}:=\sum_{\lambda} \operatorname{mult}_{V}(\lambda) \mathrm{e}^{\lambda}
$$

Since the weights $\lambda$ are linear functions on the Cartan subalgebra $\mathfrak{g}_{\circ}$ of $\mathfrak{g}$, the formal exponential $\mathrm{e}^{\lambda}-$ and hence also the character $\chi_{V}-$ can be regarded as a function on $\mathfrak{g}_{\circ}$, i.e. $\mathrm{e}^{\lambda}: \mathfrak{g}_{\circ} \rightarrow \mathbb{C}$, $h \mapsto \mathrm{e}^{\lambda}(h):=\exp (\lambda(h))$, where 'exp' is the ordinary exponential function. Moreover, via the invariant bilinear form the Cartan subalgebra $\mathfrak{g}_{\circ}$ can be identified with its dual space, i.e. with the weight space $\mathfrak{g}_{\circ}^{\star}$. Correspondingly, by setting

$$
\mathrm{e}^{\lambda}: \quad \mu \mapsto \mathrm{e}^{\lambda}(\mu):=\exp [(\lambda, \mu)] \text { for all } \mu \in \mathfrak{g}_{\circ}^{\star},
$$

\footnotetext{
${ }^{36} \bar{\theta}$ denotes again the highest root of $\overline{\mathfrak{g}}$. The presence of the length squared of $\bar{\theta}$ implements the dependence on the normalization of the inner product on the weight space $\overline{\mathfrak{g}}_{\circ}^{\star}$ of $\overline{\mathfrak{g}}$.
} 
where $(\cdot, \cdot)$ is the inner product in weight space, one can interpret the exponentials $\mathrm{e}^{\lambda}$ and the character $\chi_{V}$ also as functions from the weight space to the complex numbers.

One of the most intriguing results in the representation theory of symmetrizable Kac-Moody algebras is the Weyl-Kac character formula which states that the character $\chi_{\Lambda}$ of an irreducible $\mathfrak{g}$-module $\mathcal{H}_{\Lambda}$ with highest weight $\Lambda$ satisfies

$$
\chi_{\Lambda}(\mu)=\frac{\sum_{w \in W} \operatorname{sign}(w) \exp [(w(\Lambda+\rho), \mu)]}{\sum_{w \in W} \operatorname{sign}(w) \exp [(w(\rho), \mu)]} .
$$

Here $W$ is the Weyl group of $\mathfrak{g}$ which, just like in the finite-dimensional case, is the group generated by reflections about the hyperplanes perpendicular to the simple roots, $\operatorname{sign}(w) \in$ $\{ \pm 1\}$ is the sign of a Weyl group element $w$, and $\rho=\sum_{i} \Lambda_{(i)}$ is the Weyl vector of $\mathfrak{g}$. The formula (3.32) is a consequence of a deep interplay between the structure of the Weyl group and the relation between irreducible and Verma modules. The character $\mathcal{X}_{\Lambda}$ of a Verma module $\mathcal{V}_{\Lambda}$ is easily obtained with the help of the Poincaré-Birkhoff-Witt theorem, which tells how to construct bases of universal enveloping algebras; from the form of such a basis it follows that each generator $E^{\alpha}$ associated to a positive $\mathfrak{g}$-root $\alpha$ contributes a geometric series in $\mathrm{e}^{-\alpha}$ to $\mathcal{X}_{\Lambda}$, and by summing these series one finds that

$$
\mathcal{X}_{\Lambda}=\mathrm{e}^{\Lambda} \cdot \prod_{\alpha>0}\left(1-\mathrm{e}^{-\alpha}\right)^{-\operatorname{mult}(\alpha)} .
$$

Moreover, irreducible characters are invariant under the Weyl group $W, \chi_{\Lambda}(w(\mu))=\chi_{\Lambda}(\mu)$, while Verma characters acquire a factor of $\operatorname{sign}(w)$. When combining these results with a linear relation that relates irreducible and Verma characters and with basic properties of the Weyl group $W$, one obtains the character formula in the form

$$
\chi_{\Lambda}=\frac{\sum_{w \in W} \operatorname{sign}(w) \mathrm{e}^{w(\Lambda+\rho)-\rho}}{\prod_{\alpha>0}\left(1-\mathrm{e}^{-\alpha}\right)^{\operatorname{mult}(\alpha)}} .
$$

Furthermore, for $\Lambda=0, \mathcal{H}_{\Lambda}$ is just the trivial one-dimensional module so that it has character $\chi_{0} \equiv 1$, and hence (3.34) implies the denominator identity

$$
\prod_{\alpha>0}\left(1-\mathrm{e}^{-\alpha}\right)^{\operatorname{mult}(\alpha)}=\sum_{w \in W} \operatorname{sign}(w) \mathrm{e}^{w(\rho)-\rho}
$$

(which is the source of many most non-trivial combinatorial identities). Upon re-inserting this result into (3.34) one arrives at the character formula in the form (3.32).

With the help of Poisson resummation, the character formula allows one to analyze the behavior of characters under modular transformations. One finds that the set of characters of irreducible highest weight modules at fixed level of every untwisted (and of some twisted) $\square$ affine Lie algebra spans a module for a unitary representation of $\mathrm{SL}_{2}(\mathbb{Z})$ - compare the Kac-Peterson formula (4.11) below. As observed after equation (3.16), the derivation $D \in \mathfrak{g}_{\circ}$ of an affine Kac-Moody algebras $\mathfrak{g}$ behaves just like $-L_{0}$. Thus the restriction of the character to the span $\mathbb{C} D$ of $D$ yields precisely the Virasoro-specialized character that I already introduced in (2.11); however, the Virasoro-specialized characters are usually not sufficient to determine the representation matrices of $\mathrm{SL}_{2}(\mathbb{Z})$.

\footnotetext{
${ }^{37}$ Among the twisted affine Lie algebras, precisely those which do have nice modular transformation properties have appeared 48 in conformal field theory.
} 
The character formula is valid for arbitrary symmetrizable Kac-Moody algebras. Untwisted affine Lie algebras are nevertheless distinguished, as the formula is then often easy to evaluate. It becomes particularly simple when $\mathfrak{g}$ is simply laced and at level 1 . In this case the Virasorospecialized character $\chi_{\Lambda}(\tau)$ is, up to an over-all power of $q=\exp (2 \pi \mathrm{i} \tau)$, just the $-r$ th power of Euler's product function $\varphi(q)=\prod_{n=1}^{\infty}\left(1-q^{n}\right)$ multiplied with a theta function for the $\overline{\mathfrak{g}}$-root lattice shifted by the highest weight. E.g. for $A_{N-1}^{(1)}$ at level one the formula reads (expressing $A_{N-1}$-weights in an orthogonal basis of a space of dimension $r+1=N$ )

$$
\chi_{\Lambda_{(j)}}(\tau)=\left(q^{1 / 24} \varphi(q)\right)^{-(N-1)} q^{-j^{2} / 2 N} \sum_{\substack{j_{1}+j_{2}+\ldots, j_{N}=j \\ j_{1}, \ldots, j_{N} \in \mathbb{Z}}} q^{\left(j_{1}^{2}+j_{2}^{2}+\cdots j_{N}^{2}\right) / 2} .
$$

for $j=1,2, \ldots, N-1$.

Let me return to the relation between Verma and irreducible modules. The maximal submodule $V_{\max }$ that must be divided out from $\mathcal{V}_{\Lambda}$ in order to obtain the irreducible module $\mathcal{H}_{\Lambda}$ is not the direct sum of the Verma modules that are 'headed' by the primitive null vectors. Rather, in $V_{\max }$ the latter can overlap (moreover, in general there also exist proper submodules which are not Verma modules). However, when $\Lambda$ is an integrable weight, the overlap between two Verma submodules $\mathcal{V}_{\mu_{1}}$ and $\mathcal{V}_{\mu_{2}}$ of $\mathcal{V}_{\Lambda}$ is a submodule of both $\mathcal{V}_{\mu_{1}}$ and $\mathcal{V}_{\mu_{2}}$ and is again obtained from primitive null vectors (now in $\mathcal{V}_{\mu_{1}}$ respectively $\mathcal{V}_{\mu_{2}}$ ). Moreover, the precise structure is governed by the Weyl group of $\mathfrak{g}$. Based on this connection one can construct a semi-infinite complex of $\mathfrak{g}$-modules and $\mathfrak{g}$-module homomorphisms, the so-called $B G G$ resolution of $\mathcal{H}_{\Lambda}$, in which each module except for the one at the right-most place, which is $\mathcal{H}_{\Lambda}$, is a direct sum of Verma modules. (The structure looks rather simple, but the proof, which works even for non-symmetrizable Kac-Moody algebras [83], is most complicated.)

\section{Lecture 4: WZW theories and coset theories}

\section{WZW Theories}

The results of Lecture 3 show in particular that an untwisted affine Lie algebra $\mathfrak{g}$ enjoys all properties that are needed for a chiral algebra of conformal field theory, except that it does not contain the Virasoro algebra $\mathcal{V i r}$. The latter shortcoming is easily remedied by just prescribing the bracket relations $\left[L_{m}, K\right]=0=\left[C, \hat{T}_{n}^{a}\right]$ and

$$
\left[L_{m}, \hat{T}_{n}^{a}\right]=-n \hat{T}_{m+n}^{a}
$$

among the generators of $\mathfrak{g}$ and $\mathcal{V} \mathfrak{i r}$. This way one arrives at the semi-direct sum of $\mathcal{V} \mathfrak{i r}$ and the centrally extended loop algebra $\hat{\mathfrak{g}}=[\mathfrak{g}, \mathfrak{g}]$ and identifies the Virasoro generator $L_{0}$ with minus the derivation $D$ of the affine algebra $\mathfrak{g}$.

While this observation does not automatically imply that one can associate a conformal field theory to any untwisted affine Lie algebra $\mathfrak{g}$, indeed there exists a class of conformal field theories with such a chiral algebra $\mathfrak{g} \oplus \mathcal{V i r}$, namely the so-called $W Z W$ (Wess-Zumino-Witten) theories [81]. These theories are characterized by the fact that their chiral symmetry algebra consists of the energy-momentum tensor $T(z)$ and the current

$$
J(z)=\sum_{m \in \mathbb{Z}} z^{-m-1} \hat{T}_{m}^{a}
$$


whose modes $L_{m}$ and $\hat{T}_{n}^{a}$ satisfy the relations (1.3), (3.15) and (4.1), together with the requirement that the Virasoro generators $L_{m}$ are of the Sugawara form, i.e.

$$
L_{m}=\frac{1}{2\left(k^{\vee}+g^{\vee}\right)} \sum_{n \in \mathbb{Z}} \bar{\kappa}_{a b}: \hat{T}_{m+n}^{a} \hat{T}_{-n}^{b}:
$$

Here the colons : : denote a normal ordering prescription (compare equation $(1.30)$ ), $k^{\vee}$ is the level of the relevant $\mathfrak{g}$-modules, and $g^{\vee}$ is the dual Coxeter number of the horizontal subalgebra $\overline{\mathfrak{g}}$ of $\mathfrak{g}$, i.e., the (properly normalized) eigenvalue of the quadratic Casimir operator in the adjoint representation of $\overline{\mathfrak{g}}$. The Sugawara formula (4.3) implies that WZW theories are governed by the representation theory of $\mathfrak{g}$, and there is no need to study the Virasoro algebra independently of $\mathfrak{g}$. Moreover, it follows from the representation theory of $\mathfrak{g}$ that I summarized in section 19 that many quantities of interest of a WZW theory can be studied entirely in terms of the finite-dimensional simple Lie algebra $\overline{\mathfrak{g}}$ and of the level $k^{\vee}$. E.g., because of the relation (4.3) the Virasoro central charge $c$ can be expressed as

$$
c\left(\mathfrak{g}, k^{\vee}\right)=\frac{k^{\vee} \operatorname{dim} \overline{\mathfrak{g}}}{k^{\vee}+g^{\vee}}
$$

Note that in a quantum field theory the factor $\left(k^{\vee}+g^{\vee}\right)^{-1}$ makes sense only if $k^{\vee}$ can be regarded as a $\mathbb{C}$-number; in other words, it is necessary that all $\mathfrak{g}$-representations appearing in a WZW theory have one and the same value of the level. For unitary theories, $k^{\vee}$ is a non-negative integer.

WZW theories also possess a Lagrangian realization (from which they in fact received their name) as euclidean principal sigma models [92, i.e. two-dimensional non-linear sigma models for which the target space is a compact simple Lie group manifold $G$ - the one whose Lie algebra is the compact real form of $\overline{\mathfrak{g}}-$ which are supplemented [110] by a Wess-Zumino [109, 90] term. The action of such a WZW sigma model is $S_{\mathrm{WZW}}=k^{\vee}\left(S_{\sigma}+S_{\mathrm{WZ}}\right)$ with

$$
S_{\sigma}=\int \frac{\mathrm{d}^{2} z}{16 \pi} \operatorname{tr}\left(\partial_{\mu} \gamma \partial^{\mu} \gamma^{-1}\right), \quad S_{\mathrm{WZ}}=\int \frac{\mathrm{d}^{3} y}{24 \pi} \epsilon^{\mu \nu \tau} \operatorname{tr}\left(\dot{\gamma}^{-1} \partial_{\mu} \dot{\gamma} \dot{\gamma}^{-1} \partial_{\nu} \dot{\gamma} \dot{\gamma}^{-1} \partial_{\tau} \dot{\gamma}\right)
$$

Here $\gamma$ takes values in $G$, and the integral in $S_{\mathrm{WZ}}$ is over a three-dimensional ball whose surface is to be identified with the compactified two-dimensional world sheet such that $\dot{\gamma}=\gamma$ on the surface (the WZ term is a total derivative, hence can also be written as a surface integral).

Sometimes one uses the term WZW theory also to refer to a somewhat more general class of models, namely those where the affine algebra $\mathfrak{g}$ is replaced by a current algebra, i.e. by a direct sum of untwisted affine algebras and of Heisenberg algebras. (The Lie bracket relations of the Heisenberg algebra, which generates the observables $\mathcal{W}$ for the theory of a free boson, and which is often also called the $\hat{\mathfrak{u}}(1)$ current algebra, were displayed in $(2.20)$. Note that this Lie algebra is not a Kac-Moody algebra.) Those models for which $\mathfrak{g}$ is just an untwisted affine algebra, and hence $\overline{\mathfrak{g}}$ is simple, are then called 'simple' WZW theories.

\section{WZW Primaries}

The primary fields of a unitary WZW theory with diagonal modular invariant are in one-to-one correspondence with the integrable highest weights $\Lambda$ of an untwisted affine Lie algebra $\mathfrak{g}$ at level $k^{\vee}$, i.e. with the dominant integral $\overline{\mathfrak{g}}$-weights $\bar{\Lambda}$ that satisfy (3.28). Only finitely many 
weights of $\overline{\mathfrak{g}}$ fulfill the condition (3.28); WZW theories are therefore rational conformal field theories.

WZW primary fields $\phi=\phi_{\Lambda}(z)$ can be characterized by the properties that (compare also equation (1.27)

$$
\left[\hat{T}_{n}^{a}, \phi_{\Lambda}(0)\right]=0 \quad \text { for } n>0 \quad \text { and } \quad\left[\hat{T}_{0}^{a}, \phi_{\Lambda}(0)\right]=\bar{T}_{(\bar{\Lambda})}^{a} \phi_{\Lambda}(0)
$$

where $\bar{T}_{(\bar{\Lambda})}^{a}$ is a shorthand for the representation matrix $\bar{R}_{\bar{\Lambda}}\left(\bar{T}^{a}\right)$. In terms of operator products, these relations correspond to

$$
\underbrace{J^{a}(z) \phi_{\Lambda}}(w, \bar{w})=(z-w)^{-1} \bar{T}_{(\bar{\Lambda})}^{a} \phi_{\Lambda}(w, \bar{w})
$$

The operator product of two current fields reads

$$
\stackrel{J^{a}(z) J^{b}}{J^{\prime}}(w)=(z-w)^{-2} \bar{\kappa}^{a b} K-(z-w)^{-1} \sum_{c} f_{c}^{a b} J^{c}(w)
$$

thus while $J^{a}$ is a primary field of the Virasoro algebra, it is not primary with respect to $\mathfrak{g}$, but rather a descendant of the identity field $\mathbf{1}$ at grade one.

The conformal dimension of a $(\mathfrak{g}-)$ primary field with highest weight $\Lambda$ is

$$
\Delta_{\Lambda}=\frac{(\bar{\Lambda}, \bar{\Lambda}+2 \bar{\rho})}{2\left(k^{\vee}+g^{\vee}\right)},
$$

with $\bar{\rho}$ the Weyl vector of $\overline{\mathfrak{g}}$. Note that $(\bar{\Lambda}, \bar{\Lambda}+2 \bar{\rho})$ is the quadratic Casimir eigenvalue of the irreducible $\overline{\mathfrak{g}}$-module that has highest weight $\bar{\Lambda}$. Conjugate primary fields have highest weights which are 'charge-conjugate' with respect to $\overline{\mathfrak{g}}$, and hence identical conformal dimensions, as they should.

The Ward identity corresponding to the zero modes of the current reads

$$
\sum_{i=1}^{p} R_{i}(\bar{x})\left\langle\varphi_{\lambda_{1}} \varphi_{\lambda_{2}} \cdots \varphi_{\lambda_{p}}\right\rangle=0
$$

where $\varphi_{\lambda_{j}}$ are arbitrary conformal fields of $\overline{\mathfrak{g}}$-weight $\lambda_{j}$ and $\bar{x}=\sum_{a} \xi_{a} \hat{T}_{0}^{a}$ is any element of $\overline{\mathfrak{g}}$. By taking $\bar{x}$ in the Cartan subalgebra of $\overline{\mathfrak{g}}$, the formula (4.10) tells us that $\left(\sum_{j=1}^{p} \lambda_{j}\right)\left\langle\varphi_{\lambda_{1}} \cdots \varphi_{\lambda_{p}}\right\rangle$ $=0$. Thus a correlation function can be non-vanishing only if it is ' $\overline{\mathfrak{g}}$-neutral', i.e. if $\sum \lambda_{j}=0$. In particular, $\left\langle\phi_{\Lambda_{1}} \cdots \phi_{\Lambda_{p}}\right\rangle \equiv 0$ for each correlation function which only involves (non-trivial) fields $\phi_{\Lambda}$ which are primary not only with respect to $\mathcal{V} \mathfrak{i r}$, but also with respect to $\mathfrak{g}$. In order to study non-vanishing correlation functions it is therefore necessary to deal also with correlators which involve secondary fields. It is, however, sufficient to allow just for 'horizontal' $\mathfrak{g}$-descendants, which are obtained from the primaries $\phi_{\Lambda}$ by acting only with zero mode currents $\hat{T}_{0}^{a}$ and which are still $\mathcal{V} \mathfrak{i r}$-primary. (An analogous complication arises whenever the zero mode subalgebra $\mathcal{W}^{0}$ of the chiral symmetry algebra is non-abelian.)

\section{Modularity, Fusion Rules and WZW Simple Currents}

As already mentioned in section 20, the irreducible characters of a WZW theory span a module 
for a unitary representation of $\mathrm{SL}_{2}(\mathbb{Z})$. The explicit form of the modular T-matrix is obtained by inserting (4.9) into the general formula (2.16). The modular S-matrix is given by the Kac-Peterson formula [79]

$$
S_{\Lambda, \Lambda^{\prime}}=\mathcal{N} \sum_{w \in \bar{W}} \operatorname{sign}(w) \exp \left[-\frac{2 \pi \mathrm{i}}{k^{\vee}+g^{\vee}}\left(w(\bar{\Lambda}+\bar{\rho}), \bar{\Lambda}^{\prime}+\bar{\rho}\right)\right]
$$

Here the summation is over the Weyl group $\bar{W}$ of the horizontal subalgebra $\overline{\mathfrak{g}}$ of $\mathfrak{g}$; the normalization $\mathcal{N}$ follows from the requirement that $S$ must be unitary. As an example, consider $\mathfrak{g}=D_{N}^{(1)}$ at level one. Then there are four primary fields corresponding to the singlet (o), vector $(\mathrm{v})$, spinor $(\mathrm{s})$, and conjugate spinor (c) representation of $D_{N}$, with conformal dimensions $\Delta_{\circ}=0, \Delta_{\mathrm{v}}=1 / 2, \Delta_{\mathrm{s}}=\Delta_{\mathrm{c}}=N / 8$, and the S-matrix reads

$$
S\left(\left(D_{N}^{(1)}\right)_{1}\right)=\frac{1}{2}\left(\begin{array}{rrrr}
1 & 1 & 1 & 1 \\
1 & 1 & -1 & -1 \\
1 & -1 & \mathrm{i}^{-N} & -\mathrm{i}^{-N} \\
1 & -1 & -\mathrm{i}^{-N} & \mathrm{i}^{-N}
\end{array}\right) .
$$

Using these explicit formulæ, it has in particular been possible to construct a large number of modular invariant combinations of characters (by methods such as simple current extensions, conformal embeddings, automorphisms of the fusion rules, or (quasi-)Galois symmetries). However, so far the classification of physical modular invariants has been completed only for $\overline{\mathfrak{g}}=\mathfrak{s l}(2)$, where an $A-D-E$ pattern emerges [21], and [62] for $\overline{\mathfrak{g}}=\mathfrak{s l}(3)$. In particular, despite much progress (see e.g. 63]) it is not yet known whether already all 'exceptional' modular invariants, which are similar to the $E_{7}$-type invariant for $\mathfrak{s l}(2)$, have been found.

Via the Verlinde formula (2.4), the Kac-Peterson formula can be employed to compute the fusion rules of WZW theories. Moreover, comparison with the character formula (3.32) shows that $S_{\Lambda, \Lambda^{\prime}}$ is essentially the character of an irreducible module of the horizontal subalgebra $\overline{\mathfrak{g}}$ evaluated at a specific rational element of the weight space $\overline{\mathfrak{g}}_{\circ}^{\star}$. By combining these observations with the unitarity of the modular S-matrix and with knowledge about the tensor products of $\overline{\mathfrak{g}}$-modules in a clever manner, one finds the following Kac-Walton formula [77, 106]

$$
\mathrm{N}_{\Lambda \Lambda^{\prime}}^{\Lambda^{\prime \prime}}=\sum_{w \in W} \operatorname{sign}(w) \operatorname{mult}_{\bar{\Lambda}^{\prime}}\left(\hat{w}\left(\bar{\Lambda}^{\prime \prime}+\bar{\rho}\right)-\bar{\rho}-\bar{\Lambda}\right)
$$

for the fusion rule coefficients $\mathrm{N}_{\Lambda \Lambda^{\prime}}{ }^{\prime \prime}$ of WZW theories. Here $\bar{\rho}$ is the Weyl vector of the horizontal subalgebra $\overline{\mathfrak{g}}, W$ is the Weyl group of $\mathfrak{g}, \hat{w}$ denotes the (affine) action of $w \in W$ that is induced on the weight space of $\overline{\mathfrak{g}}$, and $\operatorname{mult}_{\bar{\Lambda}}(\bar{\mu})$ is the multiplicity with which the weight $\bar{\mu}$ arises for the irreducible highest weight module $\mathcal{H}_{\bar{\Lambda}}$ of $\overline{\mathfrak{g}}$. Since $W$ is an infinite group, (4.13) would not be of practical use if one really had to perform the sum over all of $W$, even though only a finite number of Weyl group elements gives a non-zero contribution. Fortunately, this is not necessary, because one can express the relevant elements of $W$ through a finite algorithm as products of the (finitely many) reflections which correspond to simple $\mathfrak{g}$-roots (see e.g. Appendix A of [46]).

All simple currents of WZW theories are known [45, 35, 40]. Except for an isolated case appearing for $E_{8}^{(1)}$ at level two, they are, besides the identity field, the primary fields with highest weights $k^{\vee} \Lambda_{(i)}$, where $k^{\vee}$ is the level and $\Lambda_{(i)}$ is a fundamental weight for which the Coxeter label $a_{i}$ - the coefficient of $\bar{\alpha}^{(i)}$ in the expansion of $\bar{\theta}$ with respect to the basis of simple 
$\overline{\mathfrak{g}}$-roots - has the value $a_{i}=1$. 38 The action of such a simple current $J$ on the highest weights of the primary fields corresponds to a symmetry of the Dynkin diagram of $\mathfrak{g}$, and the monodromy charge $\mathrm{Q}_{\mathrm{J}}(\Lambda)$ is proportional to the conjugacy class of $\bar{\Lambda}$.

\section{The Knizhnik-Zamolodchikov Equation}

Recall that one obtains irreducible highest weight modules of $\mathcal{W}$ by 'setting to zero' the null vectors in the corresponding Verma module. In more field theoretical terms, this means that application of certain elements of the universal enveloping algebra $\mathfrak{U}\left(\mathcal{W}_{-}\right)$to the highest weight vector yields a vector which is not contained in the space of physical states. Inserting such an operator into a correlation function must therefore give zero. Identities for correlators that are obtained this way are called null vector equations. In WZW theories, where $\mathcal{W} \supset \mathfrak{g}$, one clearly gets null vector equations by acting with the enveloping algebra of $\mathfrak{g}_{-}$, corresponding to the null vectors (3.29). These Gepner-Witten equations are purely algebraic relations (and are thus quite different from the null vector differential equations that arise for $\mathcal{W}=\mathcal{V i r}$ when $c<1$ ) and will not be treated here.

But in WZW theories the Sugawara relation (4.3) provides an additional source for null vectors. The associated null vector equations are known as Knizhnik-Zamolodchikov equations [81]. I will formulate them for the four-point functions

$$
\mathcal{G}(z, \bar{z})=\left\langle\phi_{\Lambda_{1}}(\infty, \infty) \phi_{\Lambda_{2}}(1,1) \phi_{\Lambda_{3}}(z, \bar{z}) \phi_{\Lambda_{4}}(0,0)\right\rangle
$$

As already observed, due to the Ward identity (4.10) such correlators vanish identically when the fields $\phi_{\Lambda}$ are primary with respect to $\mathfrak{g}$, and to get non-trivial correlators one must also allow for $\left(\mathcal{V i r}\right.$-primary) horizontal descendants which arise from the $\mathfrak{g}$-primaries $\phi_{\Lambda}$ by application of the zero modes $\hat{T}_{0}^{a}$ of the current; I will denote such descendants of $\phi_{\Lambda}$ by $\phi_{\Lambda}^{\lambda}$. By $\overline{\mathfrak{g}}$-symmetry, one can then expand (4.14) as

$$
\mathcal{G}_{\Lambda_{1} \Lambda_{2} \Lambda_{3} \Lambda_{4}}(z, \bar{z}) \equiv \mathcal{G}_{\Lambda_{1} \Lambda_{2} \Lambda_{3} \Lambda_{4}}^{\lambda_{1} \lambda_{2} \lambda_{3} \lambda_{4}}(z, \bar{z})=\sum_{i=1}^{\tilde{M}} \mathcal{G}_{\Lambda_{1} \Lambda_{2} \Lambda_{3} \Lambda_{4} ; i}(z, \bar{z}) \cdot\left(\mathcal{T}_{i}\right)^{\bar{\lambda}_{1} \bar{\lambda}_{2} \bar{\lambda}_{3} \bar{\lambda}_{4}}
$$

where $\left\{\mathcal{T}_{i} \mid i=1,2, \ldots, \tilde{M}\right\}$ is a basis of invariant $\overline{\mathfrak{g}}$-tensors $\mathcal{T}_{i}$ for the tensor product representation $\bar{R}_{\bar{\Lambda}_{1}} \otimes \bar{R}_{\bar{\Lambda}_{2}} \otimes \bar{R}_{\bar{\Lambda}_{3}} \otimes \bar{R}_{\bar{\Lambda}_{4}}$ of $\overline{\mathfrak{g}}$. In this description, the specific choice of horizontal descendants

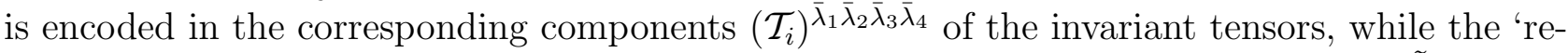
duced' functions $\mathcal{G}_{\Lambda_{1} \Lambda_{2} \Lambda_{3} \Lambda_{4} ; i}(z, \bar{z})$ only depend on the primary fields. Also, the number $\tilde{M}$ of independent invariant tensors is the $\overline{\mathfrak{g}}$-analogue of the number $M=\sum_{I_{I}} \mathrm{~N}_{A B}{ }^{I} \mathrm{~N}_{I C D}$ (cf. equation $(2.28)$ )

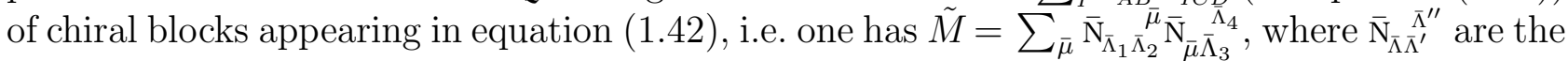
tensor product coefficients which appear in the decomposition $\bar{R}_{\bar{\Lambda}} \otimes \bar{R}_{\bar{\Lambda}^{\prime}}=\bigoplus_{\bar{\Lambda}^{\prime \prime}} \overline{\mathrm{N}}_{\bar{\Lambda} \bar{\Lambda}^{\prime}} \bar{R}_{\bar{\Lambda}^{\prime \prime}}$ of tensor products of $\overline{\mathfrak{g}}$-representations into irreducible subrepresentations. (For any value of the level, the fusion rule coefficients $\mathrm{N}_{\Lambda \Lambda^{\prime}}^{\Lambda^{\prime \prime}}=\mathrm{N}_{\Lambda \Lambda^{\prime}}{ }^{\prime \prime}\left(k^{\vee}\right)$ are majorized by the $\overline{\mathrm{N}}_{\bar{\Lambda}}{\overline{\bar{\Lambda}^{\prime}}}^{\prime \prime}$, and $\mathrm{N}_{\Lambda \Lambda^{\prime}} \bar{\Lambda}^{\prime \prime}\left(k^{\vee}\right)=\overline{\mathrm{N}}_{\bar{\Lambda} \bar{\Lambda}^{\prime}}{\overline{\bar{\Lambda}^{\prime \prime}}}^{\prime \prime}$ for $k^{\vee}$ large enough. Hence one has $\tilde{M} \geq M\left(k^{\vee}\right)$ for all $k^{\vee}$, and $\lim _{k^{\vee} \rightarrow \infty} M\left(k^{\vee}\right)=\tilde{M}$.)

\footnotetext{
${ }^{38}$ Numbering the fundamental weights as in $\left[77\right.$, these are the following. For $\overline{\mathfrak{g}}=A_{r}$, all fundamental weights qualify; their fusion rules are isomorphic to $\mathbb{Z}_{r+1}$. For $B$ and $C$ type algebras, there is a single non-trivial simple current, with $i=1$ for $B_{r}$ and $i=r$ for $C_{r}$. For $D$ type algebras, there are three non-trivial simple currents, corresponding to $i=1, r, r-1$; their fusion products are isomorphic to the multiplication of the vector, spinor, and conjugate spinor conjugacy classes of $D_{r}$. For $E_{6}$ there is a simple current of order three, with $i=1$, and its conjugate with $i=5$, and for $E_{7}$ there is a simple current of order 2 , with $i=6$.
} 
With the expansion (4.15), the Knizhnik-Zamolodchikov equation constitutes an ordinary first order matrix differential equation, reading

$$
-\frac{1}{2}\left(k^{\vee}+g^{\vee}\right) \partial \mathcal{G}_{\Lambda_{1} \Lambda_{2} \Lambda_{3} \Lambda_{4} ; i}(z, \bar{z})=\sum_{j=1}^{\tilde{M}}\left(\frac{P_{i j}}{z}+\frac{Q_{i j}}{z-1}\right) \mathcal{G}_{\Lambda_{1} \Lambda_{2} \Lambda_{3} \Lambda_{4} ; j}(z, \bar{z})
$$

where the matrices $P$ and $Q$ are given by $P=\sum_{a, b=1}^{\operatorname{dim} \overline{\mathfrak{g}}} \bar{\kappa}_{a b} \bar{R}_{\bar{\Lambda}_{3}}\left(\bar{T}^{a}\right) \otimes \bar{R}_{\bar{\Lambda}_{4}}\left(\bar{T}^{b}\right)$ and $Q=\sum_{a, b=1}^{\operatorname{dim} \overline{\mathfrak{g}}} \bar{\kappa}_{a b}$ $\bar{R}_{\bar{\Lambda}_{3}}\left(\bar{T}^{a}\right) \otimes \bar{R}_{\bar{\Lambda}_{2}}\left(\bar{T}^{b}\right)$. When the basis of invariant tensors is chosen in such a way that they correspond precisely to the various irreducible sub-representations in the tensor product $\bar{R}_{\bar{\Lambda}_{3}} \otimes \bar{R}_{\bar{\Lambda}_{4}}$, then the matrix $P$ is diagonal.

Decoupling of the system (4.16) leads to linear differential equations of order $\tilde{M}$ for the components $\mathcal{G}_{\Lambda_{1} \Lambda_{2} \Lambda_{3} \Lambda_{4} ; i}$. The solutions to these equations can be given in terms of contour integrals which generalize the contour integral formula for hypergeometric functions. This is in principle straightforward, but in practice it is rather tedious, and accordingly it has been fully worked out only for the case of $\overline{\mathfrak{g}}=\mathfrak{s l}(2)$ and for some specific isolated four-point functions for which the order $\tilde{M}$ of the differential equation is small.

\section{Coset Conformal Field Theories}

The well-established representation theory of affine Lie algebras makes WZW theories amenable to detailed study. The coset construction exploits the representation theory of affine algebras to investigate also more complicated models. The basic idea [68] is to consider embeddings $\mathfrak{h} \hookrightarrow \mathfrak{g}$, where both Lie algebras are current algebras at some level(s) $k^{\vee}$, and to analyze the difference of the two Virasoro algebras $\mathcal{V} \mathfrak{i r}_{\mathfrak{g}}$ and $\mathcal{V} \mathfrak{i r}_{\mathfrak{h}}$ (which are obtained via the Sugawara formula (4.3) for $\mathfrak{g}$ and $\mathfrak{h}$, respectively), i.e. the Lie algebra with generators

$$
L_{m}^{\mathfrak{g} / \mathfrak{h}}:=L_{m}^{\mathfrak{g}}-L_{m}^{\mathfrak{h}} .
$$

When the embedding of $\mathfrak{h}$ into $\mathfrak{g}$ is induced by an embedding $\overline{\mathfrak{h}} \hookrightarrow \overline{\mathfrak{g}}$ of the respective horizontal subalgebras, then these combinations commute with every generator $J_{n}^{a}$ of $\mathfrak{h},\left[L_{m}^{\mathfrak{g} / \mathfrak{h}}, \hat{T}_{n}^{a}\right]=0$, implying that $\left[L_{m}^{\mathfrak{g} / \mathfrak{h}}, L_{n}^{\mathfrak{g} / \mathfrak{h}}\right]=\left[L_{m}^{\mathfrak{g}}, L_{n}^{\mathfrak{g}}\right]-\left[L_{m}^{\mathfrak{h}}, L_{n}^{\mathfrak{h}}\right]$. As a consequence the generators $L_{n}^{\mathfrak{g} / \mathfrak{h}} \operatorname{span}$ a Virasoro algebra with central element $C_{\mathfrak{g} / \mathfrak{h}}=C_{\mathfrak{g}}-C_{\mathfrak{h}}$, called the coset Virasoro algebra. It is, however, still an open problem whether for any embedding $\overline{\mathfrak{h}} \hookrightarrow \overline{\mathfrak{g}}$ this prescription of a Virasoro algebra can be complemented in such a way that one arrives at a consistent conformal field theory - called the coset theory and briefly denoted by ' $(\mathfrak{g} / \mathfrak{h})_{k^{\vee}}$ ' - and if so, whether that theory is unique. To decide these questions, one must in particular construct the (maximally extended) chiral algebra $\mathcal{W}_{\mathfrak{g} / \mathfrak{h}}$ of $(\mathfrak{g} / \mathfrak{h})_{k^{\vee}}$, as well as the spectrum of the theory, i.e. tell which modules - of the coset chiral algebra $\mathcal{W}_{\mathfrak{g} / \mathfrak{h}}$, or at least of the coset Virasoro algebra $\mathcal{V i r}_{\mathfrak{g} / \mathfrak{h}}-$ appear.

As it turns out, to obtain the spectrum of the coset theory is a somewhat delicate issue. While by construction the generators of the coset Virasoro algebra act on the chiral state space of the WZW theory based on $\mathfrak{g}$, i.e. on the direct $\operatorname{sum} \mathcal{H}_{\mathfrak{g}}=\bigoplus_{\Lambda} \mathcal{H}_{\Lambda}$ of all inequivalent irreducible highest weight modules $\mathcal{H}_{\Lambda}$ of $\mathfrak{g}$ at level $k^{\vee}, \mathcal{H}_{\mathfrak{g}}$ is not the state space $\mathcal{H}_{\mathfrak{g} / \mathfrak{h}}$ of the coset theory. The crucial observation is that due to $\left[\mathfrak{h}, \mathcal{V} \mathfrak{i r}_{\mathfrak{g} / \mathfrak{h}}\right]=0$, retaining the full state space $\mathcal{H}_{\mathfrak{g}}$ would imply that the coset theory would possess (infinitely many) primary fields of zero conformal dimension other than the identity field, namely all the currents $J^{a}(z)$ of the subalgebra $\mathfrak{h}$ and 
all their $\mathfrak{h}$-descendants. In a field theoretic language this means that - in the full theory, not just accidentally in some approximation to it - the vacuum is not unique. 39 This disaster is avoided by requiring that these fields act trivially on $\mathcal{H}_{\mathfrak{g} / \mathfrak{h}}$, i.e. by imposing the gauge principle that $\mathcal{H}_{\mathfrak{g}}$-vectors which differ only by the action of $\mathfrak{U}(\mathfrak{h})$ merely provide different descriptions of the same physical situation and hence represent one and the same vector in $\mathcal{H}_{\mathfrak{g} / \mathfrak{h}}$. In short, the elements of $\mathcal{H}_{\mathfrak{g} / \mathfrak{h}}$ are $\mathfrak{U}(\mathfrak{h})$-orbits of vectors in $\mathcal{H}_{\mathfrak{g}}$ rather than individual vectors of $\mathcal{H}_{\mathfrak{g}}$.

Concretely, the restriction to $\mathfrak{U}(\mathfrak{h})$-orbits is implemented by decomposing the $\mathfrak{g}$-modules $\mathcal{H}_{\Lambda}$ into $\mathfrak{h}$-modules $\mathcal{H}_{\Lambda^{\prime}}$ as

$$
\mathcal{H}_{\Lambda}=\bigoplus_{\Lambda^{\prime}} \mathcal{H}_{\left(\Lambda ; \Lambda^{\prime}\right)} \otimes \mathcal{H}_{\Lambda^{\prime}}
$$

and the branching spaces $\mathcal{H}_{\left(\Lambda ; \Lambda^{\prime}\right)}$ appearing here are natural candidates for the irreducible modules of the coset theory. In terms of characters, this corresponds to regarding the branching functions $b_{\left(\Lambda ; \Lambda^{\prime}\right)}$ which appear in the decomposition

$$
\chi_{\Lambda}^{\mathfrak{g}}(\tau)=\sum_{\Lambda^{\prime}} b_{\left(\Lambda ; \Lambda^{\prime}\right)}(\tau) \chi_{\Lambda^{\prime}}^{\mathfrak{h}}(\tau)
$$

of the characters $\chi_{\Lambda}^{\mathfrak{g}}$ of $\mathfrak{g}$ with respect to the characters $\chi_{\Lambda^{\prime}}^{\mathfrak{h}}$ of $\mathfrak{h}$ as the characters of the coset theory.

It follows immediately from (4.19) that branching functions have a definite behavior under modular transformations, namely the same as the irreducible characters of the tensor product $\mathfrak{g} \oplus \mathfrak{h}^{*}$ of the WZW theory based on $\mathfrak{g}$ and a putative conformal field theory (called the complement of the $\mathfrak{h}$ theory [100]) which behaves just like the WZW theory based on the affine Lie algebra $\mathfrak{h}$ except that, as indicated by the notation '* , it carries the complex conjugate representation of $\mathrm{SL}_{2}(\mathbb{Z})$. (Note that if $S$ and $T$ generate a unitary representation of $\mathrm{SL}_{2}(\mathbb{Z})$, then so do $S^{*}$ and $T^{*}$.)

\section{Field Identification}

As it turns out, even after fixing the gauge symmetry $\mathfrak{h}$ there still remain severe problems; they can be attributed to the fact that in general the redundancy symmetry of the coset theory is larger than the obvious gauge symmetry $\mathfrak{h}$. It is instructive to observe this in the example of the critical Ising model, which can be realized with $\mathfrak{h}=A_{1}^{(1)}$ at level 2 embedded into $\mathfrak{g}=A_{1}^{(1)} \oplus A_{1}^{(1)}$ at levels 1 . This model is a rational theory with $c=\frac{1}{2}$ (more generally, by taking levels $m+2,1$ and $m+1$, respectively, one obtains the whole minimal series (1.21) of $\mathcal{V} \mathfrak{i r}$ ); hence its spectrum follows already from the representation theory of the Virasoro algebra. Namely, there are three primary fields, and comparing their conformal dimensions $\Delta=\bar{\Delta}$ with those of the WZW primaries one finds that each of them can be realized by two distinct pairs $\left(\Lambda ; \Lambda^{\prime}\right)$ of weights:

\begin{tabular}{lccc}
\hline \multicolumn{1}{c}{ Field } & & $\Delta$ & $\left(\Lambda ; \Lambda^{\prime}\right)$ \\
\hline identity field & $\mathbf{1}$ & 0 & $(0,0 ; 0),(1,1 ; 2)$ \\
order parameter & $\sigma$ & $1 / 16$ & $(0,1 ; 1),(1,0 ; 1)$ \\
energy operator & $\psi$ & $1 / 2$ & $(1,1 ; 0),(0,0 ; 2)$ \\
\hline
\end{tabular}

\footnotetext{
39 Note that at the level of the fusion ring, the vacuum gives the unital element, which must be unique.

40 Here and below I denote quantities referring to the subalgebra $\mathfrak{h}$ by the same symbol as the corresponding quantities for $\mathfrak{g}$, but with a prime added. In particular $\Lambda$ and $\Lambda^{\prime}$ stand for integrable highest weights of $\mathfrak{g}$ and $\mathfrak{h}$, respectively.
} 
Thus each field expected from the representation theory of $\mathcal{V} \mathfrak{i r}$ seems to appear twice. On the other hand, half of the possible pairs $\left(\Lambda ; \Lambda^{\prime}\right)$, namely those with $\Lambda_{1}+\Lambda_{2}+\Lambda^{\prime} \in 2 \mathbb{Z}+1$, do not correspond to any field of the theory at all; inspection shows that these pairs possess vanishing branching functions.

This situation generalizes for arbitrary coset theories. First, typically there are selection rules, i.e. the branching functions for certain pairs $\left(\Lambda ; \Lambda^{\prime}\right)$ vanish. This observation is in itself not too disturbing, as one just has to make sure to find all selection rules. with each selection rule there also comes a redundancy, i.e. non-vanishing branching functions for distinct pairs $\left(\Lambda ; \Lambda^{\prime}\right)$ turn out to be identical. In particular the putative vacuum sector seems to occur several times. By the same argument which forced us to divide out the action of the subalgebra $\mathfrak{h}$, one learns that this degeneracy cannot be interpreted as the multiple appearance of a corresponding primary field in the spectrum (in particular, it would then not be possible to obtain the required modular transformation properties). Rather, the correct interpretation is that a primary field of the coset theory is not associated to an individual pair $\left(\Lambda ; \Lambda^{\prime}\right)$, but rather to an appropriate equivalence class $\left[\left(\Lambda ; \Lambda^{\prime}\right)\right]$ of such pairs. This prescription has been termed field identification (which is a bit of a misnomer, since it is pairs of labels rather than conformal fields that get identified).

Both selection rules and field identification can be described conveniently via the concept of the identification group $G_{\mathrm{id}}\left[100\right.$. The elements $\left(\mathrm{J} ; \mathrm{J}^{\prime}\right)$ of $G_{\mathrm{id}}$ are simple currents of the tensor product theory $\mathfrak{g} \oplus \mathfrak{h}^{*}$ of zero conformal dimension $\Delta_{\mathrm{J}}-\Delta_{\mathrm{J}^{\prime}}$; they act on pairs $\left(\Lambda ; \Lambda^{\prime}\right)$ via the fusion product. The selection rules are equivalent to the vanishing of the monodromy charges

$$
Q_{\left(\mathrm{J} ; \mathrm{J}^{\prime}\right)}\left(\left(\Lambda ; \Lambda^{\prime}\right)\right):=Q_{\mathrm{J}}(\Lambda)-Q_{\mathrm{J}^{\prime}}\left(\Lambda^{\prime}\right)
$$

of any allowed branching function with respect to all $\left(\mathrm{J} ; \mathrm{J}^{\prime}\right) \in G_{\text {id }}$; here $Q_{\mathrm{J}}(\Lambda)$ is the combination $Q_{\mathrm{J}}(\Lambda)=\Delta_{\Lambda}+\Delta_{\mathrm{J}}-\Delta_{\mathrm{J} \star \Lambda}$ (see equation (2.25)) of conformal weights. Moreover, the equivalence classes in the field identification are precisely the orbits

$$
\left[\left(\Lambda ; \Lambda^{\prime}\right)\right]:=\left\{\left(\Upsilon ; \Upsilon^{\prime}\right) \mid \Upsilon=\mathrm{J} \Lambda, \Upsilon^{\prime}=\mathrm{J}^{\prime} \Lambda^{\prime} \text { for some }\left(\mathrm{J} ; \mathrm{J}^{\prime}\right) \in G_{\mathrm{id}}\right\}
$$

of $G_{\text {id }}$. Provided that all $G_{\text {id }}$-orbits have a common length, taking one branching function out of each $G_{\text {id }}$-orbit and combining them diagonally yields a modular invariant spectrum. In this case the modular S-matrix is given by

$$
S_{\left[\left(\Lambda ; \Lambda^{\prime}\right)\right],\left[\left(\Upsilon ; \Upsilon^{\prime}\right)\right]}^{\mathfrak{g} / \mathfrak{h}}=S_{\Lambda, \Upsilon}\left(S_{\Lambda^{\prime}, \Upsilon^{\prime}}^{\prime}\right)^{*},
$$

where $\left(\Lambda ; \Lambda^{\prime}\right)$ and $\left(\Upsilon ; \Upsilon^{\prime}\right)$ are arbitrary representatives of the orbits $\left[\left(\Lambda ; \Lambda^{\prime}\right)\right]$ and $\left[\left(\Upsilon ; \Upsilon^{\prime}\right)\right]$, respectively, i.e. is obtained by restricting the S-matrix $S \otimes\left(S^{\prime}\right)^{*}$ of $\mathfrak{g} \oplus \mathfrak{h}^{*}$ to orbits.

\section{Fixed Points}

The next degree of generality, and difficulty, is reached when $G_{\text {id }}$-orbits with different sizes

\footnotetext{
41 But still, while empirically for most coset theories all selection rules come from conjugacy class selection rules for the embedding $\overline{\mathfrak{h}} \hookrightarrow \overline{\mathfrak{g}}$, so far no prescription for enumerating all selection rules for every coset theory is known. Another possible reason for the vanishing of branching functions is the occurrence of additional null states in the Verma modules. This indeed happens for conformal embeddings, for which by definition 5.58 , the coset central charge vanishes, and presumably also for the closely related maverick [26] cosets. For all conformal embeddings, the level of $\mathfrak{g}$ is $k^{\vee}=1$, while $k^{\vee}=2$ for all known maverick cosets.
} 
appear. (Of course, all sizes are divisors of the size of the orbit through $(0 ; 0)$, on which $G_{\text {id }}$ acts freely, i.e. of the order $N=\left|G_{\text {id }}\right|$ of $G_{\text {id }}$.) Orbits of non-maximal size are referred to as fixed points. As soon as fixed points are present, taking precisely one representative out of each orbit $\left[\left(\Lambda ; \Lambda^{\prime}\right)\right]$ does not give a modular invariant spectrum. On the other hand, the sesquilinear combination

$$
Z=\sum_{\left[\Lambda ; \Lambda^{\prime}\right]: Q=0}\left|G_{\left(\Lambda ; \Lambda^{\prime}\right)}\right| \cdot\left|\sum_{\left(\mathrm{J} ; \mathrm{J}^{\prime}\right) \in G_{\mathrm{id}} / G_{\left(\Lambda ; \Lambda^{\prime}\right)}} b_{\left(\mathrm{J} ; \mathrm{J}^{\prime}\right) \star\left(\Lambda ; \Lambda^{\prime}\right)}\right|^{2}
$$

of (non-zero) branching functions is modular invariant. Here $G_{\left(\Lambda ; \Lambda^{\prime}\right)}$ denotes the stabilizer subgroup $G_{\left(\Lambda ; \Lambda^{\prime}\right)} \subseteq G_{\text {id }}$ which consists of those elements of $G_{\text {id }}$ that leave $\left(\Lambda ; \Lambda^{\prime}\right)$ invariant. Now because of $G_{(0 ; 0)}=\{(0 ; 0)\},\left|G_{\text {id }}\right|^{2}$ copies of the identity orbit $[(0 ; 0)]$ appear in (4.24), so that one would like to divide (4.24) by this factor. Regarding the branching functions $b_{\left(\Lambda ; \Lambda^{\prime}\right)}$ that contribute to (4.24) as irreducible $(\mathfrak{g} / \mathfrak{h})_{k^{\vee}}$-characters would then lead to the conclusion that fractional multiplicities occur in $Z$, which does not allow for any sensible interpretation of $Z$ as a partition function.

The difficulty in interpreting $Z$ (4.24) constitutes the fixed point problem of coset theories. This is indeed a severe problem, as it seems to prevent one from gaining control over the coset theory $(\mathfrak{g} / \mathfrak{h})_{k} \vee$ by completely understanding it in terms of the underlying WZW theories based on $\mathfrak{g}$ and $\mathfrak{h}$. The solution to this problem (see [48] or, for a condensed exposition, [50]; earlier work is summarized in [100) shows, however, that $(\mathfrak{g} / \mathfrak{h})_{k} \vee$ is in fact still fully controlled by the affine algebras $\mathfrak{g}$ and $\mathfrak{h}$, provided that one implements additional novel structures associated to Kac-Moody algebras, the so-called twining characters and orbit Lie algebras. The basic observation is that the coset modules $\mathcal{H}_{\left(\Lambda ; \Lambda^{\prime}\right)}$ for fixed points are not irreducible and hence a fixed point resolution into irreducible subspaces must be performed. (For the full two-dimensional theory obtained by combining its two chiral halves, this means that less states are present than one would naively expect.) These submodules turn out to be the simultaneous eigenspaces of certain finite order automorphism of $\mathcal{H}_{\left(\Lambda ; \Lambda^{\prime}\right)}$ that are associated to every $\left(\mathrm{J} ; \mathrm{J}^{\prime}\right) \in G_{\left(\Lambda ; \Lambda^{\prime}\right)}$ and hence are labelled by the group-characters $\Psi$ of $G_{\left(\Lambda ; \Lambda^{\prime}\right)}$; their characters can be related to the branching functions for a coset construction of orbit Lie algebras via Fourier transformation with respect to the abelian group of $G_{\left(\Lambda ; \Lambda^{\prime}\right)}$-characters. This results in particular in a formula which expresses the S-matrix of $(\mathfrak{g} / \mathfrak{h})_{k^{\vee}}$ as

$$
S_{\left(\left[\left(\Lambda ; \Lambda^{\prime}\right)\right], \Psi\right),\left(\left[\left(\Upsilon ; \Upsilon^{\prime}\right)\right], \tilde{\Psi}\right)}^{\mathfrak{g} / \mathfrak{h}}=\frac{\left|G_{\mathrm{id}}\right|}{\left|G_{\left(\Lambda ; \Lambda^{\prime}\right)}\right| \cdot\left|G_{\left(\Upsilon ; \Upsilon^{\prime}\right)}\right|} \sum_{\substack{\left(\mathrm{J} ; \mathrm{J}^{\prime}\right) \in \\ G_{\left(\Lambda ; \Lambda^{\prime}\right)} \cap G_{\left(\Upsilon ; \Upsilon^{\prime}\right)}}} \overline{\Psi\left(\left(\mathrm{J} ; \mathrm{J}^{\prime}\right)\right)} S_{\left(\Lambda ; \Lambda^{\prime}\right),\left(\Upsilon ; \Upsilon^{\prime}\right)}^{\left[\mathrm{J} ; \mathrm{J}^{\prime}\right]} \tilde{\Psi}\left(\left(\mathrm{J} ; \mathrm{J}^{\prime}\right)\right)
$$

in terms of the group characters $\Psi$ of stabilizer subgroups of $G_{\text {id }}$ and of certain modular transformation matrices $S^{\left[\mathrm{J} ; \mathrm{J}^{\prime}\right]}$ which are products of S-matrices of the relevant orbit Lie algebras.

In closing this last regular section, I would like to reiterate the remark made earlier that while in principle it is desirable to work with observable quantities only, the use of non-observable objects often proves to be convenient. The situation at hand provides an outstanding example for such an approach, in that extremely non-trivial effects can be detected without even knowing precisely what the observables $\mathcal{W}_{\mathfrak{g} / \mathfrak{h}}$ (except for the subalgebra $\mathcal{V i r}_{\mathfrak{g} / \mathfrak{h}}$ ) look like.

\section{Addenda}




\section{Omissions}

Lack of time forced me to omit a number of topics which should definitely occur in a decent introduction to conformal field theory and to Kac-Moody algebras. To partially compensate this shortcoming, I give a brief list of some of these issues and point out a few relevant references. Concerning citations, I decided to sacrifice historical accuracy for up-to-dateness, so I usually refer either to reviews or to the most recent publications I am aware of, which can serve as a guide to further literature. (This remark applies likewise to most of the references that I already mentioned in the main text.)

\section{Lecture 1:}

- more on the representation theory of the Virasoro algebra 61, 102, 97];

- classification and properties of chiral algebras [19, 108];

- vertex operator algebras 66, 78];

- more on chiral blocks and chiral vertex operators [2, 88, 23];

- conformal field theory and higher genus Riemann surfaces [88, 8, 31];

- the $C^{*}$-algebraic approach to conformal field theory [36, 58, 107, 15];

- 'logarithmic' conformal theories 60, 34.

\section{Lecture 2:}

- more on duality, crossing symmetry, and polynomial equations [2, 88];

- the representation theory and the classification of fusion rings [38, 43, 27];

- the rôle of quantum groups, or more general, quantum symmetries [2, 101, 44, 94];

- classification of modular invariants [82, 64, 47, 65];

- free fermion [69, 42] and orbifold [6, 80, 18 conformal field theories;

- Galois symmetry [20, 47, 51];

- more on the classification of $c=1$ and related conformal field theories [56, 33] and on the Coulomb gas description of $c<1$ theories [57, 97].

\section{Lecture 3:}

- more on Lie algebras with triangular decomposition [77, 54, 86, 52];

- realizations of affine Lie algebra representations via free fermions or via vertex operators 66, 77, 25, 67], and through more complicated free field constructions [24;

- character formulæ and combinatorial identities [77, 16];

- generalized Kac-Moody algebras and their rôle in string theory 66, 73].

\section{Lecture 4:}

- the affine-Virasoro construction which generalizes the Sugawara formula [71, 72];

- Lagrangian formulations of WZW and coset theories [74, 96, 103];

- Knizhnik-Zamolodchikov-Bernard equations [31, 28, 76];

- WZW theories which have fractional level [85, 55, 91] or are based on non-reductive Lie algebras [32, 3];

- Hamiltonian reduction of WZW theories [30, 61, 17];

- the relevance of Knizhnik-Zamolodchikov equations to moduli spaces of flat connections, to quasi-Hopf algebras and to integrable systems [76, 1]. 


\section{Outlook}

It is often claimed (implicitly or explicitly) that in conformal field theory all interesting problems are already solved. This statement is not entirely correct (as is e.g. indicated by the large number of recent publications quoted above); some examples of open questions are the following.

- A complete understanding of the rôle of modular invariance, in particular in view of the presence of similar structures in arbitrary rational two-dimensional quantum field theories (cf. e.g. 95, 8, 89]);

- a mathematically rigorous proof of the Verlinde formula for general conformal field theories, not just for (diagonal) WZW theories;

- structural information on fusion rules of non-quasirational two-dimensional field theories (e.g. existence of vector space bases rather than only topological bases), and the possible description of such theories as deformations or limits of quasirational theories;

- the quantum field-theoretic interpretation of non-unitary, and in particular of logarithmic conformal field theories (from a quantum field theoretic perspective it is puzzling that nonunitary theories can be treated in much the same setting as unitary ones; e.g. $\mathcal{H}$ is often fully reducible, even though positivity of an inner product can no longer be invoked);

- the classification of modular invariants for all WZW theories - as well as deeper insight into its guiding principles (note that even the $A-D-E$ pattern that arises for $\overline{\mathfrak{g}}=\mathfrak{s l}(2)$ essentially emerges in a technical rather than a conceptual manner);

- the rôle of 'apparent singularities' that appear in the differential equations for chiral blocks [41] as possible parameters in the classification of rational conformal field theories;

- simple current and Galois symmetries of braiding and fusing matrices, and a complete analysis of the behavior of fusing matrices under 'tetrahedral' transformations in the presence of fusion rule coefficients larger than one;

- a more substantial classification of modular fusion rings;

- an efficient algorithm for solving the polynomial equations for prescribed fusion rules;

- a description of conformal field theory on surfaces with boundaries [22, 93] at the same level of sophistication as for theories on closed surfaces;

- a complete description of the space of physical states for arbitrary coset theories, in particular a characterization and enumeration of all maverick cosets. 


\section{Glossary}

In the following list of keywords the quoted numbers refer to the section(s) in which the term is introduced or plays a rôle.

affine Lie algebra

annihilation operator

anti-holomorphic chiral algebra

apparent singularity

$A$-type modular invariant

BGG resolution

bootstrap

braiding matrix

$C^{*}$-algebraic approach

Cartan matrix

Cartan subalgebra

center of a conformal field theory

central element

central extension

channel

character

chiral algebra

chiral block

chiral half

chiral vertex operator

conformal anomaly

conformal dimension

conformal embedding

conformal field

conformal spin

conformal weight

conjugate field

contour integral

contraction

coroot

correlation function

coset construction

coset theory

creation operator

critical points

crossing symmetry

cross-ratio

current

current algebra

denominator identity

derivation

descendant

diagonal modular invariant

dual Coxeter number

Dynkin diagram
16

3

2

14

11

20

5,6

9

1

15

15

13

2,16

17

9

10,20

2

$7,9,14$

2

7

2,4

3

26

5

11

3

7

5,6

6

15

7

25

25

3

1

9

7

22

21

20

16

5

11

21

15 energy-momentum tensor $\quad 5$

energy operator 3

family 5

field 5

field identification 26

fixed point resolution $\quad 13,27$

formal variable 5

Fourier-Laurent coefficient 5

four-point function $\quad 9,14$

free boson $\quad 12$

fundamental weight $\quad 15$

fusing matrix 9

fusion algebra 8

fusion ring $\quad 8,13$

fusion rule coefficient 8

fusion rules 8

generalized Cartan matrix $\quad 15$

generating function 5

grade $\quad 3,5$

Heisenberg algebra $\quad 12$

hexagon equation 9

highest weight module $\quad 3,19$

highest weight vector 3

holomorphic chiral algebra 2

identification group 26

identity field 5

insertion point 7

irreducible highest weight module 3

Kac-Moody algebra $\quad 15,16$

Kac-Peterson formula 23

Kac-Walton formula 23

Knizhnik-Zamolodchikov equation 24

Laurent coefficient 5

Lie algebra 2

locally nilpotent 2

loop algebra 17

Lüscher-Mack theorem 2

maverick coset 26

maximal weight 19

minimal model $\quad 4,12$

Möbius transformation $\quad 4$

mode 5

modular group $\quad 11$

modular S-matrix $\quad 11$

modular T-matrix 11 


\begin{tabular}{|c|c|c|c|}
\hline modular transformation & 11 & S-matrix & 11 \\
\hline module & 3 & spectrum & 4 \\
\hline monodromy charge & 13 & spectrum condition & \\
\hline naturality & 4 & stabilizer subgroup & 27 \\
\hline normal ordering & 5,6 & state-field correspondence & \\
\hline null vector & 3,19 & state space & \\
\hline observable & 2 & string theory & 1,4 \\
\hline operator product algebra & 6 & Sugawara form & 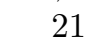 \\
\hline operator product coefficient & 6 & superselection sector & \\
\hline operator product expansion & 6 & symmetrizable Kac-Moody algebra & 1 \\
\hline orbifold & 12 & T-matrix & 11 \\
\hline order of a simple current & 13 & triangular decomposition & 3,18 \\
\hline particle contents & 1 & twisted affine Lie algebra & 16,17 \\
\hline partition function & 10,11 & twisted boundary conditions & 17 \\
\hline pentagon equation & 9 & unitary representation & \\
\hline perturbation theory & 1 & untwisted affine Lie algebra & 16,17 \\
\hline physical state & 3 & vacuum expectation value & 5,6 \\
\hline polynomial equations & 9 & vacuum sector & \\
\hline primary field & 5 & vacuum state & \\
\hline projective transformation & 4 & Verma module & 3,19 \\
\hline projective Ward identity & 7 & vertex operator algebra & 5,6 \\
\hline quantum field theory & 1 & Virasoro algebra & \\
\hline quasi-primary field & 5 & Virasoro-specialized character & 10 \\
\hline quasi-primary vector & 4 & Ward identity & \\
\hline quasi-rational conformal field theory & 8 & weight & 3,19 \\
\hline radial ordering & 6 & weight space & 15 \\
\hline rational conformal field theory & 4,8 & weight system & 10 \\
\hline Riemann monodromy problem & 14 & Weyl group & 15 \\
\hline Riemann scheme & 14 & Weyl-Kac character formula & 20 \\
\hline root & 15 & Weyl vector & 20 \\
\hline secondary field & 5 & Wightman field theory & 1,2 \\
\hline sector & 4 & Witt algebra & \\
\hline simple current & 13 & WZW theory & \\
\hline simple current extension & 13 & zero mode subalgebra & \\
\hline simple root & 15 & & \\
\hline
\end{tabular}

\section{Acknowledgements.}

I am grateful to K. Blaubär, B. Haßler, C. Schweigert and P. Vecsernyés for suggesting various improvements to the manuscript. 


\section{References}

[1] A.Yu. Alekseev, A. Recknagel, and V. Schomerus, Generalization of the Knizhnik-Zamolodchikov equations, preprint hep-th/9610066

[2] L. Alvarez-Gaumé, C. Gómez, and G. Sierra, Topics in conformal field theory, in: The Physics and Mathematics of Strings, L. Brink et al., eds. (World Scientific, Singapore 1990), p. 16

[3] H. Arfaei and S. Parvizi, WZNW models from non-standard bilinear forms, preprint hepth/9601068

[4] S. Axelrod, S. Della Pietra, and E. Witten, Geometric quantization of Chern-Simons theory, J. Diff. Geom. 33 (1991) 787

[5] F.A. Bais and P. Bouwknegt, A classification of subgroup truncations of the bosonic string, Nucl. Phys. B 279 (1987) 561

[6] P. Bantay, Algebraic aspects of orbifold models, Int. J. Mod. Phys. A 9 (1994) 1443

[7] P. Bantay, Simple current extensions and mapping class group representations, preprint hepth/9611124

[8] P. Bantay and P. Vecsernyés, Mapping class group representations and generalized Verlinde formula, preprint hep-th/9506186, to appear in Int. J. Mod. Phys. A

[9] A. Beauville, Vector bundles on curves and generalized theta functions, preprint alg-geom/9404001

[10] A. Beauville and Y. Laszlo, Conformal blocks and generalized theta functions, Commun. Math. Phys. 164 (1994) 385

[11] A.A. Belavin, A.M. Polyakov, and A.B. Zamolodchikov, Infinite conformal symmetry in two-dimensional quantum field theory, Nucl. Phys. B 241 (1984) 333

[12] M. Blau and G. Thompson, Derivation of the Verlinde formula from Chern-Simons theory and the G/G model, Nucl. Phys. B 408 (1993) 345

[13] B. Blok and S. Yankielowicz, Riemann monodromy problem and conformal field theories, Nucl. Phys. B 321 (1989) 717

[14] R. Blumenhagen, M. Flohr, A. Kliem, W. Nahm, A. Recknagel, and R. Varnhagen, $\mathcal{W}$-algebras with two and three generators, Nucl. Phys. B 361 (1991) 255

[15] J. Böckenhauer, An algebraic formulation of level one Wess-Zumino-Witten models, Rev. Math. Phys. 8 (1996) 925

[16] R.E. Borcherds, Automorphic forms on $O_{s+2,2}(\mathbf{R})$ and infinite products, Invent. math. 120 (1995) 161

[17] A. Boresch, K. Landsteiner, W. Lerche, and A. Sevrin, Superstrings from Hamiltonian reduction, Nucl. Phys. B 436 (1995) 609

[18] L. Borisov, M.B. Halpern, and C. Schweigert, Systematic approach to cyclic orbifolds, preprint hep-th/9701061, to appear in Int. J. Mod. Phys. A

[19] P. Bouwknegt and K. Schoutens, $\mathcal{W}$ symmetry in conformal field theory, Phys. Rep. 223 (1993) 183

[20] E. Buffenoir, A. Coste, J. Lascoux, P. Degiovanni, and A. Buhot, Precise study of some number fields and Galois actions occurring in conformal field theory, Ann. Inst. Poincaré 63 (1995) 41

[21] A. Cappelli, C. Itzykson, and J.-B. Zuber, The A-D-E classification of minimal and $A_{1}^{(1)}$ conformal invariant theories, Commun. Math. Phys. 113 (1987) 1 
[22] J.L. Cardy, Conformal invariance and surface critical behavior, Nucl. Phys. B 240 [FS12] (1984) 514

[23] E. Cremmer, J.-L. Gervais, and J.-F. Roussel, The genus-zero bootstrap of chiral vertex operators in Liouville theory, Nucl. Phys. B 413 (1994) 244

[24] J. de Boer, and L. Feher An explicit construction of Wakimoto realizations of current algebras, Mod. Phys. Lett. A 11 (1996) 1999

[25] L. Dolan, P. Goddard, and P. Montague, Conformal field theories, representations and lattice constructions, Commun. Math. Phys. 179 (1996) 61

[26] D.C. Dunbar and K.G. Joshi, Maverick examples of coset conformal field theories, Mod. Phys. Lett. A 8 (1993) 2803

[27] W. Eholzer, On the classification of modular fusion algebras, J. Math. Phys. 172 (1995) 623

[28] F. Falceto and K. Gawędzki, Unitarity of the Knizhnik-Zamolodchikov-Bernard connection and the Bethe ansatz for the elliptic Hitchin systems, Commun. Math. Phys. 183 (1997) 267

[29] G. Faltings, A proof for the Verlinde formula, J. Algebraic Geom. 3 (1994) 347

[30] L. Fehér, L. O'Raifeartaigh, P. Ruelle, I. Tsutsui, and A. Wipf, On Hamiltonian reductions of the Wess-Zumino-Novikov-Witten theories, Phys. Rep. 222 (1992) 1

[31] G. Felder and C. Wieczerkowski, Conformal blocks on elliptic curves and the Knizhnik-Zamolodchikov-Bernard equations, Commun. Math. Phys. 176 (1996) 163

[32] J. Figueroa-O'Farrill and S. Stanciu, Nonreductive WZW models and their CFTs, Nucl. Phys. B 458 (1996) 137

[33] M. Flohr, $\mathcal{W}$-algebras, new rational models and completeness of the $c=1$ classification, Commun. Math. Phys. 157 (1993) 179

[34] M. Flohr, On fusion rules in logarithmic conformal field theories, preprint hep-th/9605151

[35] P. Forgács, Z. Horváth, L. Palla, and P. Vecsernyés, Higher level Kac-Moody representations and rank reduction in string models, Nucl. Phys. B 308 (1988) 477

[36] K. Fredenhagen, K.-H. Rehren, and B. Schroer, Superselection sectors with braid group statistics and exchange algebras, II: Covariance properties, Rev. Math. Phys. [spec. issue] (1992) 111

[37] I.B. Frenkel, J. Lepowsky, and A. Meurman, Vertex Operator Algebras and the Monster (Academic Press, New York 1988)

[38] J. Fröhlich and T. Kerler, Quantum Groups, Quantum Categories and Quantum Field Theory [Lecture Notes in Mathematics 1542] (Springer Verlag, Berlin 1993)

[39] J. Fuchs, Operator algebra from fusion rules: The infinite number of Ising theories, Nucl. Phys. B 328 (1989) 585

[40] J. Fuchs, Simple WZW currents, Commun. Math. Phys. 136 (1991) 345

[41] J. Fuchs, Operator algebra from fusion rules II: Implementing apparent singularities, Nucl. Phys. B 386 (1992) 343

[42] J. Fuchs, Affine Lie Algebras and Quantum Groups (Cambridge University Press, Cambridge 1992)

[43] J. Fuchs, Fusion rules in conformal field theory, Fortschr. Phys. 42 (1994) 1

[44] J. Fuchs, A.Ch. Ganchev, and P. Vecsernyés, Rational Hopf algebras: Polynomial equations, gauge fixing, and low-dimensional examples, Int. J. Mod. Phys. A 10 (1995) 3431

[45] J. Fuchs and D. Gepner, On the connection between WZW and free field theories, Nucl. Phys. B 294 (1987) 30 
[46] J. Fuchs, A.N. Schellekens, and C. Schweigert, Galois modular invariants of WZW models, Nucl. Phys. B 437 (1995) 667

[47] J. Fuchs, A.N. Schellekens, and C. Schweigert, Quasi-Galois symmetries of the modular S-matrix, Commun. Math. Phys. 176 (1996) 447

[48] J. Fuchs, A.N. Schellekens, and C. Schweigert, The resolution of field identification fixed points in diagonal coset theories, Nucl. Phys. B 461 (1996) 371

[49] J. Fuchs, A.N. Schellekens, and C. Schweigert, A matrix $S$ for all simple current extensions, Nucl. Phys. B 473 (1996) 323

[50] J. Fuchs, A.N. Schellekens, and C. Schweigert, preprint hep-th/9612093, to appear in the Proceedings of the XXIth International Colloquium on Group Theoretical Methods in Physics, H.-D. Doebner and W. Scherer, eds.

[51] J. Fuchs and C. Schweigert, WZW fusion rings in the limit of infinite level, preprint hepth/9609124, to appear in Commun. Math. Phys.

[52] J. Fuchs and C. Schweigert, Symmetries, Lie Algebras and Representations (Cambridge University Press, Cambridge 1997)

[53] D.B. Fuks, Cohomology of Infinite-dimensional Lie Algebras (Consultants Bureau, New York 1986)

[54] W. Fulton and J. Harris, Representation Theory, a First Course (Springer Verlag, New York 1991)

[55] P. Furlan, A.Ch. Ganchev, R.R. Paunov, and V.B. Petkova, Solutions of the Knizhnik-Zamolodchikov equation with rational isospins and the reduction to the minimal models, Nucl. Phys. B 394 (1993) 665

[56] P. Furlan, R.R. Paunov, and I.T. Todorov, Extended U(1) conformal field theories and $\mathbf{Z}_{k^{-}}$ parafermions, Fortschr. Phys. 40 (1992) 211

[57] P. Furlan, G. Sotkov, and I.T. Todorov, Two-dimensional conformal quantum field theory, Riv. Nuovo Cim. 12 (1989) 1

[58] F. Gabbiani and J. Fröhlich, Operator algebras and conformal field theory, Commun. Math. Phys. 155 (1993) 569

[59] M.R. Gaberdiel, Fusion in conformal field theory as the tensor product of the symmetry algebra, Int. J. Mod. Phys. A 9 (1994) 4619

[60] M.R. Gaberdiel and H. Kausch, Indecomposable fusion products, Nucl. Phys. B 477 (1996) 293

[61] A.Ch. Ganchev and V.B. Petkova, Virasoro singular vectors via quantum DS reduction, Phys. Lett. B 318 (1993) 77

[62] T. Gannon, The classification of affine SU(3) modular invariant partition functions, Commun. Math. Phys. 161 (1994) 233

[63] T. Gannon, Kac-Peterson, Perron-Frobenius, and the classification of conformal field theories, preprint q-alg/9510026

[64] T. Gannon, $U(1)^{m}$ modular invariants, $N=2$ minimal models, and the quantum Hall effect, preprint hep-th/9608063

[65] T. Gannon, P. Ruelle, and M.A. Walton, Automorphism modular invariants of current algebras, Commun. Math. Phys. 179 (1996) 121

[66] R.W. Gebert and H. Nicolai, On $E_{10}$ and the DDF construction, Commun. Math. Phys. 172 (1995) 571 
[67] R.W. Gebert and H. Nicolai, An affine string vertex operator construction at arbitrary level, preprint hep-th/9608014

[68] P. Goddard, A. Kent, and D.I. Olive, Unitary representations of the Virasoro and super Virasoro algebras, Commun. Math. Phys. 103 (1986) 105

[69] P. Goddard and D.I. Olive, Kac-Moody and Virasoro algebras in relation to quantum physics, Int. J. Mod. Phys. A 1 (1986) 303

[70] R. Haag, Local Quantum Physics (Springer Verlag, Berlin 1992)

[71] M.B. Halpern, E.B. Kiritsis, N.A. Obers, and K. Clubok, Irrational conformal field theory, Phys. Rep. 265 (1996) 1

[72] M.B. Halpern and N.A. Obers, Semi-classical blocks and correlators in rational and irrational conformal field theory, Int. J. Mod. Phys. A 11 (1996) 4837

[73] J.A. Harvey and G. Moore, Algebras, BPS states, and strings, Nucl. Phys. B 463 (1996) 315

[74] K. Hori, Global aspects of gauged Wess-Zumino-Witten models, Commun. Math. Phys. 182 (1996) 1

[75] K. Intriligator, Bonus symmetry in conformal field theory, Nucl. Phys. B 332 (1990) 541

[76] D. Ivanov, Knizhnik-Zamolodchikov-Bernard equations as a quantization of nonstationary Hitchin system, preprint hep-th/9610207

[77] V.G. Kac, Infinite-dimensional Lie Algebras, third edition (Cambridge University Press, Cambridge 1990)

[78] V.G. Kac, Vertex algebras, in: New Trends in Quantum Field Theory, A.Ch. Ganchev, R. Kerner and I. Todorov, eds. (Heron Press, Sofia 1996), p. 261

[79] V.G. Kac and D.H. Peterson, Infinite-dimensional Lie algebras, theta functions and modular forms, Adv. Math. 53 (1984) 125

[80] V.G. Kac and I.T. Todorov, Affine orbifolds and rational conformal field theory extensions of $W_{1+\infty}$, preprint hep-th/9612078

[81] V.G. Knizhnik and A.B. Zamolodchikov, Current algebra and Wess-Zumino model in two dimensions, Nucl. Phys. B 247 (1984) 83

[82] M. Kreuzer and A.N. Schellekens, Simple currents versus orbifolds with discrete torsion - a complete classification, Nucl. Phys. B 411 (1994) 97

[83] S. Kumar, A Demazure character formula in arbitrary Kac-Moody setting, Invent. math. 89 (1987) 395

[84] D.C. Lewellen, Constraints for conformal field theories on the plane: reviving the conformal bootstrap, Nucl. Phys. B 320 (1989) 345

[85] P. Mathieu, P. Senechal, and M.A. Walton, Field identification in nonunitary diagonal cosets, Int. J. Mod. Phys. A 7 Suppl. 1B (1992) 731

[86] R.V. Moody and A. Pianzola, Lie Algebras With Triangular Decomposition (John Wiley, New York 1995)

[87] G. Moore and N. Seiberg, Naturality in conformal field theory, Nucl. Phys. B 313 (1989) 16

[88] G. Moore and N. Seiberg, Lectures on RCFT, in: Physics, Geometry, and Topology, H.C. Lee, ed. (Plenum, New York 1990), p. 263

[89] M. Müger, Quantum double actions on operator algebras and orbifold quantum field theories, preprint hep-th/9606175 
[90] S.P. Novikov, Multivalued functions and functionals. An analogue of the Morse theory, Sov. Math. Dokl. 24 (1981) 222

[91] J.L. Petersen, J. Rasmussen, and M. Yu, Fusion, crossing and monodromy in conformal field theory based on $S L(2)$ current algebra with fractional level, Nucl. Phys. B 481 (1996) 577

[92] A.M. Polyakov and P.B. Wiegmann, Theory of nonabelian Goldstone bosons, Phys. Lett. B 131 (1983) 121

[93] G. Pradisi, A. Sagnotti, and Ya.S. Stanev, Completeness conditions for boundary operators in $2 D$ conformal field theory, Phys. Lett. B 381 (1996) 97

[94] A. Recknagel, From path representations to global morphisms for a class of minimal models, preprint hep-th/9612178

[95] K.-H. Rehren, Braid group statistics and their superselection rules, in: The Algebraic Theory of Superselection Sectors. Introduction and Recent Results, D. Kastler, ed. (World Scientific, Singapore 1990), p. 333

[96] H. Rhedin, Gauged supersymmetric WZNW model using the BRST approach, Phys. Lett. B 373 (1996) 76

[97] A.N. Schellekens, Introduction to conformal field theory, Fortschr. Phys. 44 (1996) 605

[98] A.N. Schellekens and N.P. Warner, Conformal subalgebras of Kac-Moody algebras, Phys. Rev. D 34 (1986) 3092

[99] A.N. Schellekens and S. Yankielowicz, Extended chiral algebras and modular invariant partition functions, Nucl. Phys. B 327 (1989) 673

[100] A.N. Schellekens and S. Yankielowicz, Simple currents, modular invariants, and fixed points, Int. J. Mod. Phys. A 5 (1990) 2903

[101] V. Schomerus, Construction of field algebras with quantum symmetry from local observables, Commun. Math. Phys. 169 (1995) 193

[102] M. Schottenloher, Eine mathematische Einführung in die konforme Feldtheorie, preprint gk-mp9510/21, available as http://www.mathematik.uni-muenchen.de/ ${ }^{\sim}$ gkadmin/pr/gk_mp_9510_21.ps.gz

[103] C. Schweigert, On moduli spaces of flat connections with non-simply connected structure group, preprint hep-th/9611092

[104] R.F. Streater and A.S. Wightman, PCT, Spin and Statistics, and All That (Addison-Wesley, Reading 1989)

[105] E. Verlinde, Fusion rules and modular transformations in 2D conformal field theory, Nucl. Phys. B 300 [FS22] (1988) 360

[106] M. Walton, Fusion rules in Wess-Zumino-Witten models, Nucl. Phys. B 340 (1990) 777

[107] A. Wassermann, Operator algebras and conformal field theory III, preprint DPMMS Cambridge, available as http://www.dpmms.cam.ac.uk/home/emu/ajw/bourbaki9.ps

[108] G.M.T. Watts, contribution to these Proceedings

[109] J. Wess and B. Zumino, Consequences of anomalous Ward identities, Phys. Lett. B 37 (1971) 95

[110] E. Witten, Non-abelian bosonization in two dimensions, Commun. Math. Phys. 92 (1984) 455

[111] M. Yoshida, Fuchsian Differential Equations (Vieweg Verlag, Braunschweig 1987) 